\title{
On the Rate of Convergence to the Semi-Circular Law
}

\author{
F. Götze \\ Faculty of Mathematics \\ University of Bielefeld \\ Germany
}

\author{
A. Tikhomirov ${ }^{1}$ \\ Department of Mathematics \\ Komi Research Center of Ural Branch of RAS, \\ Syktyvkar State University \\ Russia
}

\begin{abstract}
Let $\mathbf{X}=\left(X_{j k}\right)_{j, k=1}^{n}$ denote a Hermitian random matrix with entries $X_{j k}$, which are independent for $1 \leq j \leq k \leq n$. We consider the rate of convergence of the empirical spectral distribution function of the matrix $\mathbf{X}$ to the semi-circular law assuming that $\mathbf{E} X_{j k}=0, \mathbf{E} X_{j k}^{2}=1$ and that the distributions of the matrix elements $X_{j k}$ have a uniform sub exponential decay in the sense that there exists a constant $\varkappa>0$ such that for any $1 \leq j \leq k \leq n$ and any $t \geq 1$ we have

$$
\operatorname{Pr}\left\{\left|X_{j k}\right|>t\right\} \leq A \exp \left\{-t^{\varkappa}\right\} .
$$

By means of a recursion argument it is shown that the Kolmogorov distance between the empirical spectral distribution of the Wigner matrix $\mathbf{W}=\frac{1}{\sqrt{n}} \mathbf{X}$ and the semicircular law is of order $O\left(n^{-1} \log ^{b} n\right)$ with some positive constant $b>0$.
\end{abstract}

\section{Introduction}

Consider a family $\mathbf{X}=\left\{X_{j k}\right\}, 1 \leq j \leq k \leq n$, of independent real random variables defined on some probability space $(\Omega, \mathfrak{N}, \operatorname{Pr})$, for any $n \geq 1$. Assume that $X_{j k}=X_{k j}$, for $1 \leq k<j \leq n$, and introduce the symmetric

\footnotetext{
${ }^{1}$ Research supported by SFB 701 "Spectral Structures and Topological Methods in Mathematics" University of Bielefeld. Research supported by grants RFBR N 11-0100310a and by Program of Fundamental Research Ural Division of RAS, Project 12-P-11013
} 
matrices

$$
\mathbf{W}=\frac{1}{\sqrt{n}}\left(\begin{array}{cccc}
X_{11} & X_{12} & \cdots & X_{1 n} \\
X_{21} & X_{22} & \cdots & X_{2 n} \\
\vdots & \vdots & \ddots & \vdots \\
X_{n 1} & X_{n 2} & \cdots & X_{n n}
\end{array}\right)
$$

The matrix $\mathbf{W}$ has a random spectrum $\left\{\lambda_{1}, \ldots, \lambda_{n}\right\}$ and an associated spectral distribution function $\mathcal{F}_{n}(x)=\frac{1}{n}$ card $\left\{j \leq n: \lambda_{j} \leq x\right\}, \quad x \in$ $\mathbb{R}$. Averaging over the random values $X_{i j}(\omega)$, define the expected (nonrandom) empirical distribution functions $F_{n}(x)=\mathbf{E} \mathcal{F}_{n}(x)$. Let $G(x)$ denote the semi-circular distribution function with density $g(x)=G^{\prime}(x)=$ $\frac{1}{2 \pi} \sqrt{4-x^{2}} \mathbb{I}_{[-2,2]}(x)$, where $\mathbb{I}_{[a, b]}(x)$ denotes the indicator-function of the interval $[a, b]$. We shall study the rate of convergence of $\mathcal{F}_{n}(x)$ to the semicircular law under the condition

$$
\operatorname{Pr}\left\{\left|X_{j k}\right|>t\right\} \leq A \exp \left\{-t^{\varkappa}\right\},
$$

for some $\varkappa>0, A>0$ and for any $t \geq 1$. The rate of convergence to the semi-circular law has been studied by several authors. We proved in [11] that the Kolmogorov distance between $\mathcal{F}_{n}(x)$ and the distribution function $G(x)$, $\Delta_{n}^{*}:=\sup _{x}\left|\mathcal{F}_{n}(x)-G(x)\right|$ is of order $O_{P}\left(n^{-\frac{1}{2}}\right)$ (i.e. $n^{\frac{1}{2}} \Delta_{n}^{*}$ is bounded in probability). Bai [1] and Girko [8] showed that $\Delta_{n}:=\sup _{x}\left|F_{n}(x)-G(x)\right|=$ $O\left(n^{-\frac{1}{2}}\right)$. Bobkov, Götze and Tikhomirov [7] proved that $\Delta_{n}$ and $\mathbf{E} \Delta_{n}^{*}$ have order $O\left(n^{-\frac{2}{3}}\right)$ assuming a Poincaré inequality for the distribution of the matrix elements. For the Gaussian Unitary Ensemble respectively for the Gaussian Orthogonal Ensemble, see [10] respectively [15, it has been shown that $\Delta_{n}=O\left(n^{-1}\right)$. Denote by $\gamma_{n 1} \leq \ldots \leq \gamma_{n n}$, the quantiles of $G$, i.e. $G\left(\gamma_{n j}\right)=\frac{j}{n}$. We introduce the notation $\log _{n}:=\log \log n$. Erdös, Yau and Yin [14] showed, for matrices with elements $X_{j k}$ which have a uniformly sub exponential decay, i.e. condition (1.1) holds, the following result

$$
\begin{aligned}
\left.\left.\operatorname{Pr}\left\{\exists j:\left|\lambda_{j}-\gamma_{n j}\right| \geq(\log n)^{C \log _{n}[\min \{(j, N-}-j+1\right)\right]^{-\frac{1}{3}} n^{-\frac{2}{3}}\right\} \\
\leq C \exp \left\{-(\log n)^{c \log _{n}}\right\},
\end{aligned}
$$

for $n$ large enough. It is straightforward to check that this bound implies that

$$
\operatorname{Pr}\left\{\sup _{x}\left|\mathcal{F}_{n}(x)-G(x)\right| \leq C n^{-1}(\log n)^{C \log _{n}}\right\} \geq 1-C \exp \left\{-(\log n)^{c \log _{n}}\right\} .
$$


From the last inequality it is follows that $\mathbf{E} \Delta_{n}^{*} \leq C n^{-1}(\log n)^{C} \operatorname{llog}_{n}$. In this paper we derive some improvement of the result (1.3) (reducing the power of logarithm) using arguments similar to those used in [14] and provide a self-contained proof based on recursion methods developed in the papers of Götze and Tikhomirov [11, 9] and [16. It follows from the results of Gustavsson [12] the best possible bound in the Gaussian case for the rate of convergence in probability is $O\left(n^{-1} \sqrt{\log n}\right)$. For any positive constants $\alpha>0$ and $\varkappa>0$, define the quantities

$$
l_{n, \alpha}:=\log n(\log \log n)^{\alpha} \quad \text { and } \quad \beta_{n}:=\left(l_{n, \alpha}\right)^{\frac{1}{\varkappa}+1} .
$$

The main result of this paper is the following

Theorem 1.1. Let $\mathbf{E} X_{j k}=0, \mathbf{E} X_{j k}^{2}=1$. Assume that there exists a constant $\varkappa>0$ such that inequality (1.1) holds, for any $1 \leq j \leq k \leq n$ and any $t \geq 1$. Then, for any positive $\alpha>0$ there exist positive constants $C$ and $c$ depending on $\varkappa, A$ and $\alpha$ only such that

$$
\operatorname{Pr}\left\{\sup _{x}\left|\mathcal{F}_{n}(x)-G(x)\right|>n^{-1} \beta_{n}^{4} \ln n\right\} \leq C \exp \left\{-c l_{n, \alpha}\right\} .
$$

Remark 1.2. Inequality (1.5) implies that

$$
\begin{array}{r}
\operatorname{Pr}\left\{\exists j \in\left[c \beta_{n}^{4}, n-c \beta_{n}^{4}\right]:\left|\lambda_{j}-\gamma_{n j}\right| \geq C \beta_{n}^{4}[\min \{j, N-j+1\}]^{-\frac{1}{3}} n^{-\frac{2}{3}}\right\} \\
\leq C \exp \left\{-(\log n)^{c} \log _{n}\right\} .
\end{array}
$$

For a proof of this remark see the Appendix, Subsection 9.1,

The Remark shows the rigidity of eigenvalues except for the edges $k \leq$ $C \beta_{n}^{4}$ or $k \geq n-C \beta_{n}^{4}$. (Compare with (1.2)).

We apply the result of Theorem 1.1 to study the eigenvectors of the matrix $\mathbf{W}$. Let $\mathbf{u}_{j}=\left(u_{j 1}, \ldots, u_{j n}\right)^{T}$ be eigenvectors of the matrix $\mathbf{W}$ corresponding to the eigenvalues $\lambda_{j}, j=1, \ldots, n$. We prove the following result.

Theorem 1.3. Under the conditions of Theorem 1.1, for any positive $\alpha>0$, there exist positive constants $C$ and $c$, depending on $\varkappa$ and $\alpha$ only such that

$$
\operatorname{Pr}\left\{\max _{1 \leq j, k \leq n}\left|u_{j k}\right|^{2}>\frac{\beta_{n}^{2}}{n}\right\} \leq C \exp \left\{-c l_{n, \alpha}\right\}
$$

and

$$
\operatorname{Pr}\left\{\left.\max _{1 \leq k \leq n}\left|\sum_{\nu=1}^{k}\right| u_{j \nu}\right|^{2}-\frac{k}{n} \mid>\frac{\beta_{n}^{2}}{\sqrt{n}}\right\} \leq C \exp \left\{-c l_{n, \alpha}\right\}
$$




\section{Bounds for the Kolmogorov distance between distribution functions via Stieltjes transforms}

To bound the error $\Delta_{n}^{*}$ we shall use an approach developed in previous work of the authors, see [11].

We modify the bound of the Kolmogorov distance between an arbitrary distribution function and the semi-circular distribution function via their

Stieltjes transforms obtained in [11] Lemma 2.1. For $x \in[-2,2]$ define $\gamma(x):=2-|x|$. Given $\frac{1}{2}>\varepsilon>0$ introduce the interval $\mathbb{J}_{\varepsilon}=\{x \in[-2,2]$ : $\gamma(x) \geq \varepsilon\}$ and $\mathbb{J}_{\varepsilon}^{\prime}=\mathbb{J}_{\varepsilon / 2}$. For a distribution function $F$ denote by $S_{F}(z)$ its Stieltjes transform,

$$
S_{F}(z)=\int_{-\infty}^{\infty} \frac{1}{x-z} d F(x)
$$

Proposition 2.1. Let $v>0$ and $a>0$ and $\frac{1}{2}>\varepsilon>0$ be positive numbers such that

$$
\frac{1}{\pi} \int_{|u| \leq a} \frac{1}{u^{2}+1} d u=\frac{3}{4}=: \beta
$$

and

$$
2 v a \leq \varepsilon^{\frac{3}{2}}
$$

If $G$ denotes the distribution function of the standard semi-circular law, and $F$ is any distribution function, there exist some absolute constants $C_{1}$ and $C_{2}$ such that

$$
\begin{aligned}
\Delta(F, G) & :=\sup _{x}|F(x)-G(x)| \\
& \leq 2 \sup _{x \in \mathbb{J}_{\varepsilon}^{\prime}}\left|\operatorname{Im} \int_{-\infty}^{x}\left(S_{F}\left(u+i \frac{v}{\sqrt{\gamma}}\right)-S_{G}\left(u+i \frac{v}{\sqrt{\gamma}}\right)\right) d u\right|+C_{1} v+C_{2} \varepsilon^{\frac{3}{2}} .
\end{aligned}
$$

Remark 2.2. For any $x \in \mathbb{J}_{\varepsilon}$ we have $\gamma=\gamma(x) \geq \varepsilon$ and according to condition (2.2), $\frac{a v}{\sqrt{\gamma}} \leq \frac{\varepsilon}{2}$.

For a proof of this Proposition see Subsection 9.2 of the Appendix.

Lemma 2.1. Under the conditions of Proposition 2.1, for any $V>v$ and $0<v \leq \frac{\varepsilon^{3 / 2}}{2 a}$ and $v^{\prime}=v / \sqrt{\gamma}, \gamma=2-|x|, x \in \mathbb{J}_{\varepsilon}^{\prime}$ as above, the following 
inequality holds

$$
\begin{aligned}
& \sup _{x \in \mathbb{J}_{\varepsilon}^{\prime}} \mid \int_{-\infty}^{x}\left(\operatorname{Im}\left(S_{F}\left(u+i v^{\prime}\right)-S_{G}\left(u+i v^{\prime}\right)\right) d u \mid\right. \\
& \quad \leq \int_{-\infty}^{\infty}\left|S_{F}(u+i V)-S_{G}(u+i V)\right| d u \\
& \quad+\sup _{x \in \mathbb{J}_{\varepsilon}^{\prime}}\left|\int_{v^{\prime}}^{V}\left(S_{F}(x+i u)-S_{G}(x+i u)\right) d u\right| .
\end{aligned}
$$

Proof. Let $x \in \mathbb{J}_{\varepsilon}^{\prime}$ be fixed. Let $\gamma=\gamma(x)$. Put $z=u+i v^{\prime}$. Since $v^{\prime}=$ $\frac{v}{\sqrt{\gamma}} \leq \frac{\varepsilon}{2 a}$, see (2.2), we may assume without loss of generality that $v^{\prime} \leq 4$ for $x \in \mathbb{J}_{\varepsilon}^{\prime}$. Since the functions of $S_{F}(z)$ and $S_{G}(z)$ are analytic in the upper half-plane, it is enough to use Cauchy's theorem. We can write for $x \in \mathbb{J}_{\varepsilon}^{\prime}$

$$
\int_{-\infty}^{x} \operatorname{Im}\left(S_{F}(z)-S_{G}(z)\right) d u=\operatorname{Im}\left\{\lim _{L \rightarrow \infty} \int_{-L}^{x}\left(S_{F}\left(u+i v^{\prime}\right)-S_{G}\left(u+i v^{\prime}\right)\right) d u\right\} .
$$

By Cauchy's integral formula, we have

$$
\begin{aligned}
\int_{-L}^{x}\left(S_{F}(z)-S_{G}(z)\right) d u & =\int_{-L}^{x}\left(S_{F}(u+i V)-S_{G}(u+i V)\right) d u \\
& +\int_{v^{\prime}}^{V}\left(S_{F}(-L+i u)-S_{G}(-L+i u)\right) d u \\
& -\int_{v^{\prime}}^{V}\left(S_{F}(x+i u)-S_{G}(x+i u)\right) d u .
\end{aligned}
$$

Denote by $\xi$ (resp. $\eta$ ) a random variable with distribution function $F(x)$ (resp. $G(x))$. Then we have

$$
\left|S_{F}(-L+i u)\right|=\left|\mathbf{E} \frac{1}{\xi+L-i u}\right| \leq v^{\prime-1} \operatorname{Pr}\{|\xi|>L / 2\}+\frac{2}{L},
$$

for any $v^{\prime} \leq u \leq V$. Similarly,

$$
\left|S_{G}(-L+i u)\right| \leq v^{\prime-1} \operatorname{Pr}\{|\eta|>L / 2\}+\frac{2}{L} .
$$

These inequalities imply that

$$
\left|\int_{v^{\prime}}^{V}\left(S_{F}(-L+i u)-S_{G}(-L+i u)\right) d u\right| \rightarrow 0 \quad \text { as } \quad L \rightarrow \infty,
$$

which completes the proof. 
Combining the results of Proposition 2.1 and Lemma 2.1, we get

Corollary 2.2. Under the conditions of Proposition 2.1 the following inequality holds

$$
\begin{aligned}
\Delta(F, G) & \leq 2 \int_{-\infty}^{\infty}\left|S_{F}(u+i V)-S_{G}(u+i V)\right| d u+C_{1} v+C_{2} \varepsilon^{\frac{3}{2}} \\
& +2 \sup _{x \in \mathbb{J}_{\varepsilon}^{\prime}} \int_{v^{\prime}}^{V}\left|S_{F}(x+i u)-S_{G}(x+i u)\right| d u,
\end{aligned}
$$

where $v^{\prime}=\frac{v}{\sqrt{\gamma}}$ with $\gamma=2-|x|$ and $C_{1}, C_{2}>0$ denote absolute constants.

We shall apply the last inequality. We denote the Stieltjes transform of $\mathcal{F}_{n}(x)$ by $m_{n}(z)$ and the Stieltjes transform of the semi-circular law by $s(z)$. Let $\mathbf{R}=\mathbf{R}(z)$ be the resolvent matrix of $\mathbf{W}$ given by $\mathbf{R}=\left(\mathbf{W}-z \mathbf{I}_{n}\right)^{-1}$, for all $z=u+i v$ with $v \neq 0$. Here and in what follows $\mathbf{I}_{n}$ denotes the identity matrix of dimension $n$. Sometimes we shall omit the sub index in the notation of an identity matrix. It is well-known that the Stieltjes transform of the semi-circular distribution satisfies the equation

$$
s^{2}(z)+z s(z)+1=0
$$

(see, for example, equality (4.20) in [11]). Furthermore, the Stieltjes transform of an empirical spectral distribution function $\mathcal{F}_{n}(x)$, say $m_{n}(z)$, is given by

$$
m_{n}(z)=\frac{1}{n} \sum_{j=1}^{n} R_{j j}=\frac{1}{n} \operatorname{Tr} \mathbf{R} .
$$

(see, for instance, equality (4.3) in [1]). The following often used inequalities for $\mathbf{R}_{j k}(z), 1 \leq j, k \leq n$ and $v>0$ follow via spectral representations and $\left|\lambda_{j}-z\right|^{-1} \leq v^{-1}$ as well as $\left|\lambda_{j}-z\right|^{-2}=v^{-1} \operatorname{Im}\left(\lambda_{j}-z\right)^{-1}$,

$$
\left|\mathbf{R}_{j, k}\right| \leq v^{-1}, \quad n^{-1} \sum_{j, k}\left|\mathbf{R}_{j, k}\right|^{2} \leq v^{-1} m_{n}(z)
$$

\section{Auxiliary Lemmas}

In this Section we state several Lemmas needed for the proof of the main results. The proof of these are given in the Appendix. 


\subsection{Truncation}

We consider truncated random variables $\widehat{X}_{j l}$ defined by

$$
\widehat{X}_{j l}:=X_{j l} \mathbb{I}\left\{\left|X_{j l}\right| \leq c l_{n, \alpha}^{\frac{1}{\varkappa}}\right\}-\mathbf{E} X_{j l} \mathbb{I}\left\{\left|X_{j l}\right| \leq c l_{n, \alpha}^{\frac{1}{\varkappa}}\right\} .
$$

Let $\widehat{\mathcal{F}}_{n}(x)$ denote the empirical spectral distribution function of the matrix $\widehat{\mathbf{W}}=\frac{1}{\sqrt{n}}\left(\widehat{X}_{j l}\right)$ and let $\widehat{m}_{n}(z)$ be the corresponding Stieltjes transform. Let $\sigma_{j k}^{2}=\mathbf{E}\left(\widehat{X}_{j k}\right)^{2}$. Introduce the r.v.'s $\widetilde{X}_{j k}=\sigma_{j k}^{-1} \widehat{X}_{j k}$. Consider the matrix $\widetilde{\mathbf{W}}=\frac{1}{\sqrt{n}}\left(\widetilde{X}_{j k}\right)$. Let $\widetilde{m}_{n}(z)$ denote the corresponding Stieltjes transform.

Lemma 3.1. Assuming the conditions of Theorem 1.1 there exist constants $C, c>0$ such that

$$
\operatorname{Pr}\left\{\left|m_{n}(z)-\widetilde{m}_{n}(z)\right| \geq \frac{C}{n^{2} v^{2}}\right\} \leq \exp \left\{-c l_{n, \alpha}\right\} .
$$

Remark 3.1. In what follows we shall assume that r.v.'s $X_{j l}$ satisfy the condition

$$
\left|X_{j l}\right| \leq A_{0} l_{n, \alpha}^{\frac{1}{\varkappa}}, \quad \mathbf{E} X_{j l}=0 \text { and } \mathbf{E} X_{j k}^{2}=1,
$$

for some absolute constant $A_{0}$ We shall omit the symbol $\hat{\cdot}$ in the notation of the truncated r.v.'s and corresponding characteristics of truncated matrices.

\subsection{The Key Lemma}

Denote by $\mathbb{T}=\{1, \ldots, n\}$. For any $\mathbb{A} \subset \mathbb{T}$, introduce the matrices $\mathbf{W}^{(\mathbb{A})}$, which are obtained from $\mathbf{W}$ by deleting the $j$-th row and the $j$-th column for $j \in \mathbb{A}$, and the corresponding resolvent matrix $\mathbf{R}^{(\mathbb{A})}$ defined by $\mathbf{R}^{(\mathbb{A})}:=$ $\left(\mathbf{W}^{(\mathbb{A})}-z \mathbf{I}_{n-|\mathbb{A}|}\right)^{-1}$ and let $m_{n}^{(\mathbb{A})}(z):=\frac{1}{n} \operatorname{Tr} \mathbf{R}^{(\mathbb{A})}$. Consider the index set $\mathbb{T}_{\mathbb{A}}:=\{1, \ldots, n\} \backslash\{\mathbb{A}\}$. By $\mathfrak{M}^{(\mathbb{A})}$ we shall denote $\sigma$-algebra generated by $\mathrm{r}$. v.'s $X_{r q}$ with $r, q \in \mathbb{T}_{\mathbb{A}}$.

We shall use the representation

$$
R_{j j}=\frac{1}{-z+\frac{1}{\sqrt{n}} X_{j j}-\frac{1}{n} \sum_{k, l \in \mathbb{T}_{j}} X_{j k} X_{j l} R_{k l}^{(j)}},
$$

(see, for example, equality (4.6) in [11]). We may rewrite it as follows

$$
R_{j j}=-\frac{1}{z+m_{n}(z)}+\frac{1}{z+m_{n}(z)} \varepsilon_{j} R_{j j}
$$


where $\varepsilon_{j}:=\varepsilon_{j 1}+\varepsilon_{j 2}+\varepsilon_{j 3}+\varepsilon_{j 4}$ with

$$
\begin{aligned}
& \varepsilon_{j 1}:=\frac{1}{\sqrt{n}} X_{j j}, \quad \varepsilon_{j 2}:=\frac{1}{n} \sum_{k \in \mathbb{T}_{j}}\left(X_{j k}^{2}-1\right) R_{k k}^{(j)}, \\
& \varepsilon_{j 3}:=\frac{1}{n} \sum_{k \neq l \in \mathbb{T}_{j}} X_{j k} X_{j l} R_{k l}^{(j)}, \quad \varepsilon_{j 4}:=\frac{1}{n}\left(\operatorname{Tr} \mathbf{R}^{(j)}-\operatorname{Tr} \mathbf{R}\right) .
\end{aligned}
$$

This relation immediately implies the following two equations

$$
\begin{aligned}
R_{j j}=-\frac{1}{z+} m_{n}(z) & -\sum_{\nu=1}^{3} \frac{\varepsilon_{j \nu}}{\left(z+m_{n}(z)\right)^{2}}+ \\
& +\sum_{\nu=1}^{3} \frac{1}{\left(z+m_{n}(z)\right)^{2}} \varepsilon_{j \nu} \varepsilon_{j} R_{j j}+\frac{1}{z+m_{n}(z)} \varepsilon_{j 4} R_{j j}
\end{aligned}
$$

and summing in $j=1, \ldots, n$,

$$
\begin{aligned}
m_{n}(z)= & -\frac{1}{z+m_{n}(z)}-\frac{1}{\left(z+m_{n}(z)\right)} \frac{1}{n} \sum_{j=1}^{n} \varepsilon_{j} R_{j j} \\
= & -\frac{1}{z+m_{n}(z)}-\frac{1}{\left(z+m_{n}(z)\right)^{2}} \frac{1}{n} \sum_{\nu=1}^{3} \sum_{j=1}^{n} \varepsilon_{j \nu}+ \\
& +\frac{1}{\left(z+m_{n}(z)\right)^{2}} \frac{1}{n} \sum_{\nu=1}^{3} \sum_{j=1}^{n} \varepsilon_{j \nu} \varepsilon_{j} R_{j j}+\frac{1}{z+m_{n}(z)} \frac{1}{n} \sum_{j=1}^{n} \varepsilon_{j 4} R_{j j} .
\end{aligned}
$$

Let $V_{0}=4+v_{0}$ and $v_{0}=\frac{c_{0} \beta_{n}^{4}}{n}$, where $c_{0} \geq \frac{3 \cdot 6^{8}}{\log 2}$. Without loss of generality we may assume that $n v_{0} \geq 2$, for any $n \geq 1$. We choose $c_{0}$ later. Let $v_{k}=v_{0}+\frac{k}{n^{2}}$, for $k=0, \ldots, N=4 n^{2}$. Introduce the events, for $k=0, \ldots, N$,

$$
\mathcal{A}_{k}=\left\{\omega:\left|m_{n}(z)-s(z)\right| \leq \frac{1}{2} \text {, for any } z=u+i v \text { such that } v \geq v_{k}\right\} .
$$

Furthermore, let $\mathcal{D}_{k}$, for $k=0, \ldots, N$, denote the events

$$
\mathcal{D}_{k}=\left\{\omega:\left|\varepsilon_{j}\right| \leq \gamma_{0} ; j=1, \ldots, n ; z=u+i v: v \geq v_{k}\right\},
$$

where $\gamma_{0}=\frac{3}{100}$ is an absolute constant.

Lemma 3.2. Assuming the conditions of Theorem 1.1 the following relations hold

$$
\mathcal{D}_{k} \cap \mathcal{A}_{k} \subset \mathcal{A}_{k-1}, \text { for } k=1, \ldots, N \text {. }
$$


Proof. For a proof of this Lemma see Subsection 9.4 of the Appendix.

Corollary 3.3. We have

$$
\operatorname{Pr}\left\{\mathcal{A}_{k}^{c}\right\} \leq \sum_{l=k+1}^{N} \operatorname{Pr}\left\{\mathcal{D}_{l}^{c} \cap \mathcal{A}_{l}\right\} .
$$

Proof. Note that $v_{N} \geq 4$. We have, for $z=u+i v$ with $v \geq v_{N}$

$$
\max \left\{\left|m_{n}(z)\right|,|s(z)|\right\} \leq \frac{1}{4} \text { a. s. }
$$

That means that

$$
\operatorname{Pr}\left\{\mathcal{A}_{N}\right\}=1
$$

By Lemma 3.2, we have

$$
\operatorname{Pr}\left\{\mathcal{A}_{k}^{(c)}\right\} \leq \operatorname{Pr}\left\{\left(\mathcal{D}_{k+1} \cap \mathcal{A}_{k+1}\right)^{c}\right\} \leq \operatorname{Pr}\left\{\mathcal{D}_{k+1}^{c} \cap \mathcal{A}_{k+1}\right\}+\operatorname{Pr}\left\{A_{k+1}^{c}\right\} .
$$

The claim of Corollary 4.2 now follows from 3.10 by induction.

In the following we shall systematically use (as above) for any $n \times n$ matrix $\mathbf{W}$ together with its resolvent $\mathbf{R}$, its Stieltjes transform $m_{n}$ etc. the corresponding quantities $\mathbb{W}^{(\mathbb{A})}, \mathbf{R}^{(\mathbb{A})}$ and $m_{n}^{(\mathbb{A})}$ for the corresponding sub matrix with entries $X_{j k}, j, k \notin \mathbb{A}, \mathbb{A} \subset \mathbb{T}=\{1, \ldots, n\}$.

If $\mathbb{A}=\emptyset$ we shall omit the set $\mathbb{A}$ as exponent index. Fix some integer $L$ which will be chosen later. Introduce additionally the event, for any $\mathbb{A} \subset \mathbb{T}$ such that $|\mathbb{A}| \leq L$,

$$
\mathcal{U}^{(\mathbb{A})}:=\left\{\operatorname{Im} m_{n}^{(\mathbb{A})}(z) \leq \psi_{0}+\frac{L-|\mathbb{A}|+1}{n v}\right\},
$$

where

$$
\psi_{0} \leq B_{0}, \text { for all } z=u+i v, v \geq v_{0}
$$

and

$$
\psi_{0} \geq \max \left\{\frac{1}{n v}, c \sqrt{v}\right\}, \text { for } z=u+i v, u \in[-2,2], v \geq v_{0} .
$$

The function $\psi_{0}=\psi_{0}(z)$ and the absolute constant $B_{0}$ will be chosen later.

Remark 3.2. Note that for any $z=u+i v$ the inequality

$$
\operatorname{Im} m_{n}(z)>\operatorname{Im} m_{n}^{(\mathbb{A})}(z)-\frac{|\mathbb{A}|}{n v}
$$

holds a.s. This yields, for $\mathbb{A}_{1} \subset \mathbb{A}_{2}$,

$$
\left(\mathcal{U}^{\left(\mathbb{A}_{1}\right)}\right)^{c} \subset\left(\mathcal{U}^{\left(\mathbb{A}_{2}\right)}\right)^{c} .
$$


We introduce as well the notation $\quad \kappa_{n}=\psi_{0} l_{n, \alpha}^{\frac{1}{\varkappa}}$.

\section{Lemma 3.4.}

$$
\operatorname{Pr}\left\{\mathcal{D}_{k}^{c} \cap \mathcal{A}_{k} \cap \mathcal{U}\right\} \leq \exp \left\{-c \sqrt{n v_{k} / \kappa_{n}}\right\} .
$$

Proof. For a proof of this Lemma see Subsection 9.5 of the Appendix.

Introduce now for $z=u+i v \in \mathbb{C}_{+}$

$$
\mathcal{A}_{v}=\left\{\left|m_{n}(z)-s(z)\right| \leq \frac{1}{2}, \text { for any } u \in \mathbb{R}\right\} .
$$

Applying Lemmas 3.2 and 3.4 , we get

Corollary 3.5. The following inequality holds for any $v \geq v_{0}$

$$
\operatorname{Pr}\left\{\mathcal{A}_{v}^{c} \cap \mathcal{U}\right\} \leq \exp \left\{-C \sqrt{n v / \kappa_{n}}\right\}
$$

for some positive constant $C>0$. There exists a constant $C>0$ such that

$$
\operatorname{Pr}\left\{\left\{\left|R_{j j}\right| \leq 3, \text { for any } j=1, \ldots, n\right\} \cap \mathcal{U}\right\} \leq \exp \left\{-C \sqrt{n v / \kappa_{n}}\right\} .
$$

Moreover, for $z=u+i v$ with $|u| \leq 2$ and $0<v \leq 5$, there exist a constant $\delta>0$ such that

$$
\operatorname{Pr}\left\{\left\{\left|R_{j j}\right| \geq \delta, \text { for any } j=1, \ldots, n\right\} \cap \mathcal{U}\right\} \leq \exp \left\{-C \sqrt{n v / \kappa_{n}}\right\} .
$$

Proof. Let $v_{k} \geq v \geq v_{k-1}$. By Lemma 3.2, we have

$$
\begin{aligned}
\operatorname{Pr}\left\{\mathcal{A}_{v}^{c} \cap \mathcal{U}\right\} & \leq \sum_{t=k+1}^{N} \operatorname{Pr}\left\{\mathcal{D}_{t}^{c} \cap \mathcal{A}_{t} \cap \mathcal{U}\right\} \leq n^{2} \exp \left\{-C \sqrt{n v / \kappa_{n}}\right\} \\
& \leq \exp \left\{-C^{\prime} \sqrt{n v / \kappa_{n}}\right\}
\end{aligned}
$$

with some positive constant $C^{\prime}$. This inequality and Lemma 3.4 yield inequality (3.16).

Furthermore, we note that the events $\mathcal{A}_{v}$ and $\mathcal{D}_{k}$ together imply by (9.52) that

$$
\left|R_{j j}(u+i v)\right| \leq 3
$$

On the other hand the events $\mathcal{A}_{v}$ and $\mathcal{D}_{k}$ with (3.3) imply

$$
\left|R_{j j}(u+i v)\right| \geq \frac{1}{2\left|z+m_{n}(z)\right|} \geq \frac{1}{6},
$$

for $|u| \leq 2$. This proves inequalities (3.17) and (3.18). 
Corollary 3.6. Assuming conditions of Theorem 1.1, we have

$$
\operatorname{Pr}\left\{\mathcal{D}_{k}^{c}\right\} \leq \exp \left\{-C \sqrt{n v} / l_{n, \alpha}^{\frac{2}{\varkappa}}\right\} .
$$

Proof. The result follows from the inequality

$$
\operatorname{Pr}\left\{\mathcal{D}_{k}^{c}\right\} \leq \operatorname{Pr}\left\{\mathcal{D}_{k+1}^{c} \cap \mathcal{A}_{k+1}\right\}+\operatorname{Pr}\left\{\mathcal{A}_{k+1}^{c}\right\}
$$

and Lemma 3.4 for $B_{0}=\frac{3}{2}$.

Corollary 3.7. Assuming the conditions of Theorem 1.1, we have

$$
\operatorname{Pr}\left\{\mathcal{A}_{v}\right\} \leq \exp \left\{-C \sqrt{n v} / l_{n, \alpha}^{\frac{2}{\varkappa}}\right\} .
$$

Proof. The claim follows from Corollaries 3.6 and 3.3.

\subsection{A Bound for $\sum_{l}\left|R_{j l}\right|^{2}$}

In this Section we investigate the quantity

$$
H_{m}^{(j, \mathbb{A})}=\mathbf{E}\left\{\left(\sum_{t \in \mathbb{T}_{\mathbb{A}, j}}\left|R_{j t}^{(\mathbb{A})}\right|^{2}\right)^{m} \mathbb{I}\{\mathcal{U}\} \mid \mathfrak{M}^{(j, \mathbb{A})}\right\}, \text { for } j \in \mathbb{T}_{\mathbb{A}} .
$$

In what follows we shall denote by $\Phi_{n}$ a generic non-negative random error term such that

$$
\mathbf{E} \Phi_{n} \leq \exp \left\{-c \sqrt{n v / \kappa_{n}} / \ln n\right\}
$$

for some positive constant $c>0$. Furthermore, introduce the event

$$
\mathcal{B}^{(\mathbb{A})}=\cap_{l \in \mathbb{T}_{\mathbb{A}}}\left\{C_{1} \leq\left|R_{l l}^{(\mathbb{A})}\right| \leq C_{2}\right\}
$$

for some constants $C_{1}, C_{2}$ such that

$$
\operatorname{Pr}\left\{\left(\mathcal{B}^{(\mathbb{A})}\right)^{c} \cap \mathcal{U}^{(\mathbb{A})}\right\} \leq \exp \left\{-c \sqrt{n v / \kappa_{n}}\right\} .
$$

We may apply Corollary 3.5 to the matrix $\mathbf{W}^{(\mathbb{A})}$ to prove that such constants exist. Note that, by definition of $\Phi_{n}$, for $1 \leq m \leq C \log n$,

$$
\mathbf{E}^{\frac{1}{m}} \mathbb{I}\left\{\left(\mathcal{B}^{(\mathbb{A})}\right)^{c} \cap \mathcal{U}^{(\mathbb{A})} \mid \mathfrak{M}^{(j, \mathbb{A})}\right\} \leq \Phi_{n} .
$$

Lemma 3.8. Under the conditions of Theorem 1.1 there exists a constant $C>0$ such that for any $1 \leq m \leq C \log n$

$$
\left(H_{m}^{(j, \mathbb{A})}\right)^{\frac{1}{m}} \leq C m l_{n, \alpha}^{\frac{2}{\varkappa}} v^{-1} \psi_{0}+\Phi_{n}
$$


Proof. Here and in what follows we shall consider the case $\mathbb{A}=\emptyset$ only. Otherwise consider the matrix $\mathbf{W}^{(\mathbb{A})}$ instead of $\mathbf{W}$. Applying the identity

$$
R_{j q}=-\frac{1}{\sqrt{n}} \sum_{t \in \mathbb{T}_{j}} X_{j t} R_{q t}^{(j)} R_{j j},
$$

we obtain, for $\omega \in \mathcal{B}$,

$$
\sum_{q \in \mathbb{T}_{j}}\left|R_{j q}\right|^{2} \leq \frac{C}{n} \sum_{q \in \mathbb{T}_{j}}\left|\sum_{t \in \mathbb{T}_{j, q}} X_{j t} R_{q t}^{(j)}\right|^{2} .
$$

We may write

$$
\left(H_{m}^{(j)}\right)^{\frac{1}{m}} \leq v^{-2} \operatorname{Pr}^{\frac{1}{m}}\left\{\mathcal{B}^{c} \cap \mathcal{U} \mid \mathfrak{M}^{(j)}\right\}+\mathbf{E}^{\frac{1}{m}}\left\{\mathbb{I}\{\mathcal{B} \cap \mathcal{U}\}\left(\sum_{q \in \mathbb{T}_{j, q}}\left|R_{j q}\right|^{2}\right)^{m} \mid \mathfrak{M}^{(j)}\right\} .
$$

Inequalities (3.28) and (3.29) together imply using (3.26) as well as $m=$ $\log n$ and $v \geq v_{0}$

$$
\begin{aligned}
\left(H_{m}^{(j)}\right)^{\frac{1}{m}} & \leq \Phi_{n} \\
& +\mathbb{I}\left\{\operatorname{Im} m_{n}^{(j)}(z) \leq \psi_{0}+\frac{1}{n v}\right\}\left(\mathbf{E}\left\{\left(\frac{1}{n} \sum_{q \in \mathbb{T}_{j}}\left|\sum_{r \in \mathbb{T}_{j, q}} X_{j r} R_{q r}^{(j)}\right|^{2}\right)^{m} \mid \mathfrak{M}^{(j)}\right\}\right)^{\frac{1}{m}} .
\end{aligned}
$$

Consider the random variables $Y_{r}=\frac{1}{c l_{n, \alpha}^{\frac{1}{n}}} X_{j r}$. Note that $Y_{1}, \ldots, Y_{n}$ are independent and, by Remark 3.1, $\left|Y_{r}\right| \leq 1, \quad \mathbf{E} Y_{r}=0$.

Consider the quadratic form in $Y_{1}, \ldots, Y_{n}$

$$
f\left(Y_{1}, \ldots, Y_{n}\right)=\sum_{q=1}^{n}\left(\sum_{r=1}^{n} a_{q r} Y_{r}\right)^{2}
$$

with $a_{q r}=R_{r q}^{(j)} / \sqrt{n}$. Note that $f$ is a convex function. Let $Z_{1}, \ldots, Z_{n}$ denote standard Gaussian r.v.'s. Then it follows from results of Bobkov [4], [5] (Choquet comparison of measures), that

$$
\mathbf{E}^{\frac{1}{m}}\left|f\left(Y_{1}, \ldots, Y_{n}\right)\right|^{m} \leq \mathbf{E}^{\frac{1}{m}}\left|f\left(c_{0} Z_{1}, \ldots, c_{0} Z_{n}\right)\right|^{m},
$$

were $c_{0}=\frac{\sqrt{2 \pi}}{2}$. Note that

$$
f\left(c_{0} Z_{1}, \ldots, c_{0} Z_{n}\right)=c_{0}^{2} f\left(Z_{1}, \ldots, Z_{n}\right) .
$$


For the Gaussian r.v.'s we have ([6], Theorem 3.1)

$$
\mathbf{E}^{\frac{1}{m}}\left|f\left(Z_{1}, \ldots, Z_{n}\right)\right|^{m} \leq C m \mathbf{E}\left|f\left(Z_{1}, \ldots, Z_{n}\right)\right|=C m \sum_{q=1}^{n} \sum_{r=1}^{n}\left|a_{q r}\right|^{2} .
$$

In our case

$$
\sum_{q=1}^{n} \sum_{r=1}^{n}\left|a_{q r}\right|^{2} \leq \frac{1}{n} \operatorname{Tr}\left|\mathbf{R}^{(j)}\right|^{2} \leq v^{-1}\left(\operatorname{Im} m_{n}(z)+\frac{1}{n v}\right) .
$$

Applying these inequalities, we get, using that $X_{j q}=c l_{n, \alpha}^{\frac{1}{\pi}} Y_{q}$,

$$
\left(\mathbf{E}\left\{\left(\frac{1}{n} \sum_{q \in \mathbb{T}_{j}}\left(\left|\sum_{r \in \mathbb{T}_{j, q}} X_{j r} R_{q r}^{(j)}\right|^{2}\right)^{m} \mid \mathfrak{M}^{(j)}\right\}\right)^{\frac{1}{m}} \leq C m v^{-1} l_{n, \alpha}^{\frac{2}{\varkappa}}\left(\operatorname{Im} m_{n}(z)+\frac{1}{n v}\right) .\right.
$$

Note that by definition $\psi_{0} \geq \frac{1}{n v}$. Inequalities (3.32) and (3.30) together imply

$$
\begin{aligned}
\left(H_{m}^{(j)}\right)^{\frac{1}{m}} & \leq \Phi_{n}+m l_{n, \alpha}^{\frac{2}{\varkappa}} v^{-1} \mathbb{I}\left\{\operatorname{Im} m_{n}^{(j)}(z) \leq \psi_{0}+\frac{1}{n v}\right\}\left(\operatorname{Im} m_{n}(z)+\frac{1}{n v}\right) \\
& \leq C m l_{n, \alpha}^{\frac{2}{\varkappa}} v^{-1} \psi_{0}+\Phi_{n} .
\end{aligned}
$$

Thus Lemma 3.8 is proved.

Note that for $1 \leq m \leq C \log n$

$$
\begin{aligned}
v^{-1} \mathbf{E} \operatorname{Pr}^{\frac{1}{m}}\left\{\left(\mathcal{B}^{(\mathbb{A}, l)}\right)^{c} \cap \mathcal{U}^{(\mathbb{A}, l)} \mid \mathfrak{M}^{(j, \mathbb{A})}\right\} & \leq v^{-1} \mathbf{E}^{\frac{1}{m}} \mathbb{I}\left\{\left(\mathcal{B}^{(\mathbb{A}, l)}\right)^{c} \cap \mathcal{U}^{(\mathbb{A}, l)}\right\} \\
& \leq \exp \left\{-c \sqrt{n v / \kappa_{n}} / \log n\right\}
\end{aligned}
$$

This means that

$$
v^{-1} \operatorname{EPr}^{\frac{1}{m}}\left\{\left(\mathcal{B}^{(\mathbb{A}, l)}\right)^{c} \cap \mathcal{U}^{(\mathbb{A}, l)} \mid \mathfrak{M}^{(j, \mathbb{A})}\right\} \leq \Phi_{n} .
$$

Lemma 3.9. Assuming the conditions of Theorem 1.1, there exist constants $c, C$ such that for any $1 \leq m \leq C \log n$ and for any $\mathbb{A}, j, l$ such that $l \in \mathbb{T}_{\mathbb{A}}$, 
$j \in \mathbb{T}_{\mathbb{A}, l}$ and $|\mathbb{A}| \leq C \log n$,

$$
\begin{aligned}
\mathbf{E}^{\frac{1}{m}}\left\{\left(\sum_{q \in \mathbb{T}_{\mathbb{A}, j, l}}\left|R_{q l}^{(\mathbb{A})}\right|^{2}\right)^{m} \mathbb{I}\left\{\mathcal{U}^{(\mathbb{A})}\right\} \mid \mathfrak{M}^{(\mathbb{A}, j)}\right\} \\
\leq C\left(\sum_{q \in \mathbb{T}_{\mathbb{A}, j, l}}\left|R_{q l}^{(\mathbb{A}, j)}\right|^{2}\right) \mathbf{E}\left\{\mathbb{I}\left\{\mathcal{U}^{(\mathbb{A})}\right\} \mid \mathfrak{M}^{(\mathbb{A}, j)}\right\} \\
\quad+\left(\frac{C m l_{n, \alpha}^{\frac{2}{\varkappa}}}{\sqrt{n}}\right)\left(\sum_{q \in \mathbb{T}_{\mathbb{A}, j, l}}\left|R_{q l}^{(\mathbb{A}, j)}\right|^{2}\right)^{\frac{1}{2}} \mathbf{E}\left\{\mathbb{I}\left\{\mathcal{U}^{(\mathbb{A})}\right\} \mid \mathfrak{M}^{(j)}\right\}+\Phi_{n} .
\end{aligned}
$$

Proof. For the proof of this Lemma see Subsection 9.6 of the Appendix.

\subsection{Some Auxiliary Bounds for $\sum_{j=1}^{n} \varepsilon_{j 3}$}

In this section we consider the sum

$$
\zeta=\frac{1}{n} \sum_{j=1}^{n} \zeta_{j}=\frac{1}{n} \sum_{j=1}^{n} \varepsilon_{j 3},
$$

where

$$
\zeta_{j}:=\varepsilon_{j 3}=\frac{1}{n} \sum_{r \neq q \in \mathbb{T}_{j}} X_{j r} X_{j q} R_{q r}^{(j)} .
$$

We shall introduce similar quantities to $\zeta$ for the matrix $\mathbf{W}^{(\mathbb{A})}$. More precisely,

$$
\zeta^{(\mathbb{A})}=\frac{1}{n} \sum_{l \in \mathbb{T}_{\mathbb{A}}} \zeta_{l}^{(\mathbb{A})}
$$

where

$$
\zeta_{l}^{(\mathbb{A})}=\frac{1}{n} \sum_{r \neq q \in \mathbb{T}_{\mathbb{A}, l}} X_{l r} X_{l q} R_{r q}^{(\mathbb{A}, l)} .
$$

Introduce the events, for $\mathbb{A} \subset \mathbb{T}$ such that $|\mathbb{A}| \leq L$,

$$
\begin{aligned}
& \mathcal{E}^{(\mathbb{A})}=\left\{\left|\operatorname{Im} m_{n}^{(\mathbb{A})}-s(z)\right| \leq \frac{1}{2}-\frac{|\mathbb{A}|}{n v}\right\}, \\
& \mathcal{F}_{l}^{(\mathbb{A})}=\left\{\left|\zeta_{l}^{(\mathbb{A})}\right| \leq(L-|\mathbb{A}|+1) \rho_{n}\left(\operatorname{Im} m_{n}^{(\mathbb{A}, l)}\right)^{\frac{1}{2}}\right\}, \\
& \mathcal{F}^{(\mathbb{A})}=\cap_{l \in \mathbb{T}_{\mathbb{A}}} \mathcal{F}_{l}^{(\mathbb{A})}, \quad \rho_{n}=\frac{c_{1} \sqrt{\kappa_{n}}}{\sqrt{n v}} .
\end{aligned}
$$


Introduce the events $\mathcal{G}^{(j)}=\mathcal{U}^{(j)} \cap \mathcal{E}^{(j)} \cap \mathcal{F}^{(j)}$ and $\mathcal{G}=\mathcal{U} \cap \mathcal{E} \cap \mathcal{F}$. Furthermore, we introduce the random variables

$$
\widehat{\zeta}_{l}=\zeta_{l} \mathbb{I}\{\mathcal{G}\}, \quad \widehat{\zeta}_{l}^{(j)}=\zeta_{l}^{(j)} \mathbb{I}\left\{\mathcal{G}^{(j)}\right\} .
$$

Note that,

$$
\left|\widehat{\zeta}_{l}\right| \leq \frac{C L \sqrt{\kappa_{n}}}{\sqrt{n v}}, \quad\left|\widehat{\zeta}_{l}^{(j)}\right| \leq \frac{2 C L \sqrt{\kappa_{n}}}{\sqrt{n v}}
$$

Define $\mathcal{G}^{(\mathbb{A})}$ similarly to $\mathcal{G}^{(j)}$ and write

$$
\widehat{\zeta}^{(\mathbb{A})}=\frac{1}{n} \sum_{j \in \mathbb{T}_{\mathbb{A}}} \widehat{\zeta}_{j}^{(\mathbb{A})}
$$

Lemma 3.10. Assuming the conditions of Theorem [1.1 and condition

$$
\operatorname{Pr}\left\{\left(\mathcal{U}^{(\mathbb{A})}\right)^{c}\right\} \leq \exp \left\{-c l_{n, \alpha}\right\},
$$

there exist constants $c, C>0$ such that for any $v \geq v_{0}$

$$
\operatorname{Pr}\{\zeta \neq \widehat{\zeta}\} \leq \exp \left\{-c l_{n, \alpha}\right\}
$$

Proof. For the proof of this lemma see Subsection 9.7 of the Appendix.

Recall that $\mathfrak{M}^{(\mathbb{A})}$ denote the $\sigma$-algebra generated by all $X_{p q}$ except the entries $X_{j q}$ and $X_{q j}$, for $q=1, \ldots, n$ and $j \in \mathbb{A}$, that is the rows and columns listed in $\mathbb{A}$. Note that $\zeta^{(\mathbb{A})}$ is measurable w.r.t. $\mathfrak{M}^{(\mathbb{A})}$ and independent of $X_{j r}$ for $j \in \mathbb{A}$ and any $r \in \mathbb{T}_{\mathbb{A}}$.

We consider the difference $\zeta_{l}-\zeta_{l}^{(j)}$. We shall first expand the quantity $\zeta_{l}-\zeta_{l}^{(j)}$ in terms of the r.v.'s $X_{j q}$, for $q \in \mathbb{T}_{j}$.

Note that

$$
\widehat{\zeta}_{l}-\widehat{\zeta}_{l}^{(j)}=\left(\zeta_{l}-\zeta_{l}^{(j)}\right) \mathbb{I}\{\mathcal{G}\} \mathbb{I}\left\{\mathcal{G}^{(j)}\right\}-\zeta_{l}^{(j)} \mathbb{I}\left\{\mathcal{G}^{c}\right\} \mathbb{I}\left\{\mathcal{G}^{(j)}\right\}+\zeta_{l} \mathbb{I}\{\mathcal{G}\} \mathbb{I}\left\{\left(\mathcal{G}^{(j)}\right)^{c}\right\}
$$

and write using $R_{q r}^{(l)}-R_{q r}^{(l, j)}=R_{j q}^{(l)} R_{j r}^{(l)} / R_{j j}^{(l)}$, (see as well (3.59) ),

$$
\begin{aligned}
\zeta_{l}-\zeta_{l}^{(j)} & =\frac{1}{n} \sum_{q \neq r \in \mathbb{T}_{l}} X_{l q} X_{l r} R_{q r}^{(l)}-\frac{1}{n} \sum_{q \neq r \in \mathbb{T}_{l, j}} X_{l q} X_{l r} R_{q r}^{(l, j)} \\
& =\frac{2}{n} X_{l j} \sum_{q \in \mathbb{T}_{l, j}} X_{l q} R_{q j}^{(l)}+\frac{1}{n} \sum_{q \neq r \in \mathbb{T}_{l, j}} X_{l q} X_{l r}\left(R_{q r}^{(l)}-R_{q r}^{(l, j)}\right) \\
& =\frac{2}{n} X_{l j} \sum_{q \in \mathbb{T}_{l, j}} X_{l q} R_{q j}^{(l)}+\frac{1}{n} \sum_{q \neq r \in \mathbb{T}_{l, j}} X_{l q} X_{l r} R_{j q}^{(l)} R_{j r}^{(l)}\left(R_{j j}^{(l)}\right)^{-1} \\
& =\xi_{l j}+\eta_{l j},
\end{aligned}
$$


where

$$
\xi_{l j}=\frac{1}{n} \sum_{r \neq q \in \mathbb{T}_{l, j}} X_{l r} X_{l q} R_{j q}^{(l)} R_{j r}^{(l)}\left(R_{j j}^{(l)}\right)^{-1}, \quad \eta_{l j}=\frac{2}{n} X_{l j} \sum_{q \in \mathbb{T}_{l, j}} X_{l q} R_{j q}^{(l)}
$$

Furthermore, we introduce notation

$$
\widehat{\eta}_{l j}=\eta_{l j} \mathbb{I}\{\mathcal{G}\} \mathbb{I}\left\{\mathcal{G}^{(j)}\right\}, \quad \widehat{\xi}_{l j}=\xi_{l j} \mathbb{I}\{\mathcal{G}\} \mathbb{I}\left\{\mathcal{G}^{(j)}\right\},
$$

and

$$
r_{j}=-\zeta^{(j)} \mathbb{I}\left\{\mathcal{G}^{c}\right\} \mathbb{I}\left\{\mathcal{G}^{(j)}\right\}+\zeta \mathbb{I}\{\mathcal{G}\} \mathbb{I}\left\{\left(\mathcal{G}^{(j)}\right)^{c}\right\}
$$

Then,

$$
\widehat{\zeta}-\widehat{\zeta}^{(j)}=\frac{1}{n} \sum_{l \in \mathbb{T}_{j}} \widehat{\xi}_{l j}+\frac{1}{n} \sum_{l \in \mathbb{T}_{j}} \widehat{\eta}_{l j}+r_{j}+\frac{1}{n} \widehat{\zeta}_{j} .
$$

Note that by expansion in the lth and $j$ th row

$$
R_{l j}=-\frac{1}{\sqrt{n}} \sum_{q \in \mathbb{T}_{l}} X_{l q} R_{j q}^{(l)} R_{l l}=-\frac{1}{\sqrt{n}} \sum_{q \in \mathbb{T}_{j}} X_{j q} R_{l q}^{(j)} R_{j j}
$$

Using these equalities, we rewrite $R^{(l)}$ in terms of $R$ and smaller terms via

$$
\begin{aligned}
\xi_{l j}= & \left(\frac{1}{\sqrt{n}} \sum_{q \in \mathbb{T}_{l}} X_{l q} R_{j q}^{(l)}-\frac{1}{\sqrt{n}} X_{l j} R_{j j}^{(l)}\right)\left(-R_{l j} R_{l l}^{-1}-\frac{1}{\sqrt{n}} X_{l j} R_{j j}^{(l)}\right)\left(R_{j j}^{(l)}\right)^{-1} \\
& -\frac{1}{n} \sum_{q \in \mathbb{T}_{l, j}} X_{l q}^{2}\left(R_{j q}^{(l)}\right)^{2}\left(R_{j j}^{(l)}\right)^{-1} \\
= & \left(-R_{l j} R_{l l}^{-1}-\frac{1}{\sqrt{n}} X_{l j} R_{j j}^{(l)}\right)^{2}\left(R_{j j}^{(l)}\right)^{-1}-\frac{1}{n} \sum_{q \in \mathbb{T}_{l, j}} X_{l q}^{2}\left(R_{j q}^{(l)}\right)^{2}\left(R_{j j}^{(l)}\right)^{-1}
\end{aligned}
$$

The last relation in (3.46) and the representation (3.45) together imply

$$
\begin{aligned}
\left|\xi_{l j}\right| \leq 2 \mid \frac{1}{n} \sum_{q \in \mathbb{T}_{j}} & \left.X_{j q} R_{l q}^{(j)}\right|^{2}\left|R_{j j}^{(l)}\right|^{-1}\left|R_{j j}\right|^{2}\left|R_{l l}\right|^{-2} \\
& +\frac{2 l_{n, \alpha}^{\frac{2}{\varkappa}}}{n}\left|R_{j j}^{(l)}\right|+\frac{C l_{n, \alpha}^{\frac{2}{\varkappa}}}{n} \sum_{q \in \mathbb{T}_{l, j}}\left|R_{j q}^{(l)}\right|^{2}\left|R_{j j}^{(l)}\right|^{-1} .
\end{aligned}
$$


Lemma 3.11. Under the conditions of Theorem 1.1 there exist constants $C, c>0$ such that for any subset $\mathbb{A} \subset \mathbb{T}$ with $|\mathbb{A}| \leq C \log n$,

$$
\mathbf{E}^{\frac{1}{m}}\left\{\left|\widehat{\xi}_{l j}^{(\mathbb{A})}\right|^{m} \mid \mathfrak{M}^{(j, \mathbb{A})}\right\} \leq \frac{C m l_{n, \alpha}^{\frac{2}{\varkappa}}}{n v}\left(v \sum_{t \in \mathbb{T}_{j, l}}\left|R_{l t}^{(j, \mathbb{A})}\right|^{2} \mathbb{I}\left\{\mathcal{U}^{(j)}\right\}+\psi_{0}\right)+\Phi_{n}
$$

Proof. We consider the case $\mathbb{A}=\emptyset$ only. In general we consider $\mathbf{W}^{(\mathbb{A})}$ instead of $\mathbf{W}$. Note that for $\omega \notin \mathcal{U}$ we have $\widehat{\xi}_{l j}^{(\mathbb{A})}=0$. By relation (3.47), for $\omega \in \mathcal{G} \cap \mathcal{G}^{(j)} \cap \mathcal{B}^{(j)} \cap \mathcal{B}^{(l)} \cap \mathcal{B}$, (where $\mathcal{B}^{(\mathbb{A})}$ has been defined in $(3.25)$ ), we have

$$
\left|\xi_{l j}\right| \leq C\left|\frac{1}{\sqrt{n}} \sum_{q \in \mathbb{T}_{j}} X_{j q} R_{l q}^{(j)}\right|^{2}+\frac{C l_{n, \alpha}^{\frac{2}{\varkappa}}}{n v} \psi_{0}^{2}+\frac{C l_{n, \alpha}^{\frac{2}{\varkappa}}}{n} \sum_{q \in \mathbb{T}_{l, j}}\left|R_{j q}^{(l)}\right|^{2} .
$$

From here, applying Rosenthal's inequality, it follows

$$
\begin{aligned}
& \mathbf{E}^{\frac{1}{m}}\left\{\left|\widehat{\xi}_{l j}\right|^{m} \mathbb{I}\left\{\mathcal{B}^{(j)} \cap \mathcal{B}^{(l)} \cap \mathcal{B}\right\} \mid \mathfrak{M}^{(j)}\right\} \\
& \leq \frac{C m l_{n, \alpha}^{\frac{2}{\varkappa}}}{n}\left(\sum_{q \in \mathbb{T}_{j}}\left|R_{l q}^{(j)}\right|^{2} \mathbb{I}\left\{\mathcal{U}^{(j)}\right\}+v^{-1} \psi_{0}\right. \\
& \left.+\mathbf{E}^{\frac{1}{m}}\left\{\left(\sum_{q \in \mathbb{T}_{j, l}}\left|R_{j q}^{(l)}\right|^{2}\right)^{m} \mathbb{I}\left\{\mathcal{U}^{(l)} \cap \mathcal{B}^{(l)}\right\} \mid \mathfrak{M}^{(j)}\right\}\right) .
\end{aligned}
$$

We use here that $\psi_{0} \leq B_{0}$. Applying now Lemma 3.8 we obtain

$$
\mathbf{E}^{\frac{1}{m}}\left\{\left|\widehat{\xi}_{l j}\right|^{m} \mathbb{I}\{\mathcal{B}\} \mid \mathfrak{M}^{(j)}\right\} \leq \frac{C m l_{n, \alpha}^{\frac{2}{\varkappa}}}{n}\left(\sum_{q \in \mathbb{T}_{j, l}}\left|R_{l q}^{(j)}\right|^{2} \mathbb{I}\left\{\mathcal{U}^{(j)}\right\}+v^{-1} \psi_{0}\right)+\Phi_{n} .
$$

Thus Lemma 3.11 is proved.

Lemma 3.12. Under the conditions of Theorem 1.1 there exist constants $C, c>0$ such that

$$
\mathbf{E}^{\frac{1}{m}}\left\{\left|\widehat{\eta}_{l j}\right|^{m} \mid \mathfrak{M}^{(j)}\right\} \leq \frac{m l_{n, \alpha}^{\frac{2}{\varkappa}}}{n}\left(\left(\sum_{q \in \mathbb{T}_{j l}}\left|R_{l q}^{(j)}\right|^{2}\right)^{\frac{1}{2}} \mathbb{I}\left\{\mathcal{U}^{(j)}\right\}+\psi_{0}\right)+\Phi_{n} .
$$

Proof. We use equality (3.45) again. We write

$$
\begin{aligned}
\eta_{l j} & =\frac{X_{l j}}{\sqrt{n}}\left(-R_{l j} R_{l l}^{-1}-\frac{X_{l j}}{\sqrt{n}} R_{j j}^{(l)}\right) \\
& =\frac{X_{l j}}{\sqrt{n}}\left(R_{j j} \frac{1}{\sqrt{n}} \sum_{q \in \mathbb{T}_{j}} X_{j q} R_{q l}^{(j)} R_{l l}^{-1}-\frac{X_{l j}}{\sqrt{n}} R_{j j}^{(l)}\right) .
\end{aligned}
$$


We may write

$$
\mathbf{E}\left\{\left|\widehat{\eta}_{l j}\right|^{m} \mid \mathfrak{M}^{(j)}\right\} \leq \mathbf{E}\left\{\left|\widehat{\eta}_{l j}\right|^{m} \mathbb{I}\{\mathcal{B}\} \mid \mathfrak{M}^{(j)}\right\}+\mathbf{E}\left\{\left|\widehat{\eta}_{l j}\right|^{m} \mathbb{I}\left\{\mathcal{B}^{(c)}\right\} \mid \mathfrak{M}^{(j)}\right\} .
$$

Applying Rosenthal's inequality, we get

$$
\mathbf{E}^{\frac{1}{m}}\left\{\left|\widehat{\eta}_{l j}\right|^{m} \mathbb{I}\{\mathcal{B}\} \mid \mathfrak{M}^{(j)}\right\} \leq \frac{m l_{n, \alpha}^{\frac{2}{\varkappa}}}{n}\left(\sum_{q \in \mathbb{T}_{j l}}\left|R_{l q}^{(j)}\right|^{2}\right)^{\frac{1}{2}} \mathbb{I}\left\{\operatorname{Im} m_{n}^{(j)} \leq \psi_{0}\right\}+\frac{C l_{n, \alpha}^{\frac{2}{\varkappa}}}{n} .
$$

Moreover,

$$
\mathbf{E}^{\frac{1}{m}}\left\{\left|\widehat{\eta}_{l j}\right|^{m} \mathbb{I}\left\{\mathcal{B}^{c}\right\} \mid \mathfrak{M}^{(j)}\right\} \leq \frac{C l_{n, \alpha}^{\frac{2}{\varkappa}}}{\sqrt{n v}} \mathbf{E}^{\frac{1}{m}}\left\{\mathbb{I}\left\{\mathcal{B}^{c} \cap \mathcal{U}\right\} \mid \mathfrak{M}^{(j)}\right\} \leq \Phi_{n} .
$$

Thus the Lemma is proved.

Lemma 3.13. Under the conditions of Theorem 1.1 there exists a constant $C>0$ such that for the term $r_{j}$ in (3.44) we have

$$
\mathbf{E}^{\frac{1}{m}}\left\{\left|r_{j}\right|^{m} \mid \mathfrak{M}^{(j)}\right\} \leq \frac{C m \sqrt{\kappa_{n}}}{\sqrt{n v}} \mathbb{I}\left\{\left(\mathcal{G}^{(j)}\right)^{c}\right\}+\Phi_{n}
$$

Proof. For the proof of this lemma see Subsection 9.8 of the Appendix.

We now conclude the bound of the r.h.s of (3.44).

Lemma 3.14. There exist constants $c, C>0$ such that for any $1 \leq m \leq$ $C \log n$,

$$
\begin{aligned}
& \sum_{l \in \mathbb{T}_{j}} \mathbf{E}^{\frac{1}{m}}\left\{\left|\widehat{\zeta}_{l}-\widehat{\zeta}_{l}^{(j)}\right|^{m} \mid \mathfrak{M}^{(j)}\right\} \\
& \quad \leq \frac{C m \kappa_{n}}{n v}+\frac{C m \sqrt{\kappa_{n}}}{\sqrt{n v}} \mathbb{I}\left\{\left(\mathcal{G}^{(j)}\right)^{c}\right\}+\Phi_{n}
\end{aligned}
$$

Proof. In order to prove this claim it is enough to sum the right hand sides of the inequalities in Lemmas 3.11, 3.48 and 3.13 for $l \in \mathbb{T}_{j}$.

We consider now the functions $g^{(\mathbb{A})}(s)=\mathbf{E} \exp \left\{s \widehat{\zeta}^{(\mathbb{A})}\right\}$ and $g(s)=g^{(\emptyset)}(s)$.

Lemma 3.15. There exist constants $c, C>0$ such that, for $s \leq c n v /\left(\kappa_{n} \log n\right)$,

$$
g^{(\mathbb{A})}(s) \leq C^{|\mathbb{A}|} g(s) .
$$


Proof. We shall prove inequality (3.51) for $|A|=1$. The general case follows by induction. Let $\mathbb{A}=\{j\}$. Note that

$g(s)=\mathbf{E} \exp \left\{s\left(\widehat{\zeta}-\widehat{\zeta}^{(j)}\right)\right\} \exp \left\{\widehat{\zeta}^{(j)}\right\}=\mathbf{E}\left(\mathbf{E}\left\{\exp \left\{s\left(\widehat{\zeta}-\widehat{\zeta}^{(j)}\right)\right\} \mid \mathfrak{M}^{(j)}\right\}\right) \exp \left\{\widehat{\zeta}^{(j)}\right\}$

Applying Jensen's inequality, we get

$$
g(s) \geq \mathbf{E}\left(\exp \left\{s \mathbf{E}\left\{\widehat{\zeta}-\widehat{\zeta}^{(j)} \mid \mathfrak{M}^{(j)}\right\}\right\} \exp \left\{\widehat{\zeta}^{(j)}\right\}\right)
$$

By Lemma 3.14, we have

$$
V_{j}=\mathbf{E}\left\{\left|\widehat{\zeta}-\widehat{\zeta}^{(j)}\right| \mid \mathfrak{M}^{(j)}\right\} \leq \frac{C \kappa_{n}}{n v}+\frac{C \sqrt{\kappa_{n}}}{\sqrt{n v}} \mathbb{I}\left\{\left(\mathcal{G}^{(j)}\right)^{c}\right\}+\Phi_{n}
$$

This implies that

$$
\exp \left\{-s V_{j}\right\} \geq \exp \left\{-\frac{s \kappa_{n}}{n v}\right\} \exp \left\{-\Phi_{n}\right\} \exp \left\{-\frac{C \sqrt{\kappa_{n}}}{\sqrt{n v}} \mathbb{I}\left\{\left(\mathcal{G}^{(j)}\right)^{c}\right\}\right\},
$$

Note that for any r.v.'s $a, b$ such that $a b=0$, we have $\exp \{a+b\}=\exp \{a\}+$ $\exp \{b\}-1$. Since $\mathbb{I}\left\{\left(\mathcal{G}^{(j)}\right)^{c}\right\} \widehat{\zeta}^{(j)}=0$, we get

$$
\begin{aligned}
\exp \left\{-s V_{j}\right\} \exp \left\{s \widehat{\zeta}_{j}\right\} \geq \exp \left\{-\frac{s \kappa_{n}}{n v}\right\} \exp \left\{-\Phi_{n}\right\}\left[\exp \left\{s \widehat{\zeta}^{(j)}\right\}\right. \\
\left.+\exp \left\{-\frac{s \sqrt{\kappa_{n}}}{\sqrt{n v}} \mathbb{I}\left\{\left(\mathcal{G}^{(j)}\right)^{c}\right\}\right\}-1\right]
\end{aligned}
$$

From here it follows that

$$
\begin{gathered}
\exp \left\{-s V_{j}\right\} \exp \left\{s \widehat{\zeta}_{j}\right\} \geq \exp \left\{-\frac{s \kappa_{n}}{n v}\right\} \exp \left\{-\Phi_{n}\right\} \exp \left\{s \widehat{\zeta}^{(j)}\right\} \\
-\exp \left\{-\frac{s \kappa_{n}}{n v}\right\} \exp \left\{-\Phi_{n}\right\}
\end{gathered}
$$

Note that

$$
\exp \left\{-\Phi_{n}\right\} \geq \exp \left\{-c_{0}\right\} \mathbb{I}\left\{\Phi_{n} \leq c_{0}\right\}
$$

and, for $0<s \leq C n v / \kappa_{n}$,

$$
\exp \left\{-\frac{s \kappa_{n}}{n v}\right\} \exp \left\{-\Phi_{n}\right\} \geq \exp \left\{-c_{1}\right\} \mathbb{I}\left\{\Phi_{n} \leq c_{0}\right\} .
$$

Combining these bounds with inequality (3.52) and inequality (3.38), we get, for $0 \leq s \leq c^{\prime} n v /\left(\kappa_{n} \log n\right)$,

$$
\begin{aligned}
g(s) & \geq \exp \left\{-c_{1}\right\} \mathbf{E} \mathbb{\{}\left\{\Phi_{n} \leq c_{0}\right\} \exp \left\{\widehat{\zeta}_{j}\right\} \\
& \geq \exp \left\{-c_{1}\right\} \mathbf{E} \exp \left\{\widehat{\zeta}_{j}\right\}-\exp \left\{-c_{1}\right\} \exp \left\{\frac{c s \sqrt{\kappa_{n}}}{\sqrt{n v}}\right\} \mathbf{E} \mathbb{I}\left\{\Phi_{n}>c_{0}\right\} \\
& \geq \exp \left\{-c_{1}\right\} \mathbf{E} \exp \left\{\widehat{\zeta}_{j}\right\}-\exp \left\{-c_{1}\right\} \exp \left\{\frac{c c^{\prime} \sqrt{n v}}{\sqrt{\kappa_{n}} \log n}\right\} \mathbf{E} \mathbb{I}\left\{\Phi_{n}>c_{0}\right\} .
\end{aligned}
$$


It is easy to see that

$$
\mathbf{E} \mathbb{I}\left\{\Phi_{n}>c_{0}\right\} \leq \exp \left\{-C \sqrt{n v / \kappa_{n}} / \log n\right\} .
$$

We may chose the constant $c^{\prime}$ small enough that

$$
\exp \left\{\frac{c c^{\prime} \sqrt{n v}}{\sqrt{\kappa_{n}} \log n}\right\} \mathbf{E} \mathbb{I}\left\{\Phi_{n}>c_{0}\right\} \leq \exp \left\{-c l_{n, \alpha}\right\} .
$$

Without loss of generality we may assume that $g(s) \leq 1$. From here it follows that for $0 \leq s \leq c n v /\left(\kappa_{n} \log n\right)$

$$
\mathbf{E} \exp \left\{\widehat{\zeta}_{j}\right\} \leq C g(s)
$$

The last bound completes the proof. Thus Lemma 3.15 is proved.

\subsection{Some additional bounds}

We consider in this Section the quantities

$$
\alpha_{l j}^{(\mathbb{A}, t)}=\xi_{l j}^{(\mathbb{A})}-\xi_{l j}^{(\mathbb{A}, t)}
$$

and

$$
\gamma_{l j}^{(\mathbb{A}, t)}=\eta_{l j}^{(\mathbb{A})}-\eta_{l j}^{(\mathbb{A}, t)}
$$

where $t \in \mathbb{T}_{\mathbb{A}}, j \in \mathbb{T}_{\mathbb{A}, t}$, and $l \in \mathbb{T}_{\mathbb{A}, t, j}$. For $p \neq r \in \mathbb{T}_{\mathbb{A}, q}$ and $q \in \mathbb{T}_{\mathbb{A}}$ we have

$$
R_{q r}^{(\mathbb{A})}-R_{q r}^{(\mathbb{A}, t)}=R_{q t}^{(\mathbb{A})} R_{r t}^{(\mathbb{A})}\left(R_{t t}^{(\mathbb{A})}\right)^{-1},
$$

(see, for instance [13], Lemma 3.2, formula (3.6)) and may represent

$$
\alpha_{l j}^{(\mathbb{A}, t)}=\widetilde{\alpha}_{l j}^{(\mathbb{A}, t)}+\widehat{\alpha}_{l j}^{(\mathbb{A}, t)}
$$

where

$$
\begin{aligned}
& \widetilde{\alpha}_{l j}^{(\mathbb{A}, t)}=\frac{1}{n} X_{l t} \sum_{q \in \mathbb{T}_{\mathbb{A}, l, t}} X_{l q} R_{q j}^{(\mathbb{A}, l)} R_{t j}^{(\mathbb{A}, l)}\left(R_{j j}^{(\mathbb{A}, l)}\right)^{-1}, \\
& \widehat{\alpha}_{l j}^{(\mathbb{A}, t)}=\widehat{\alpha}_{1 l j}^{(\mathbb{A}, t)}+\widehat{\alpha}_{2 l j}^{(\mathbb{A}, t)}+\widehat{\alpha}_{3 l j}^{(\mathbb{A}, t)}
\end{aligned}
$$

and

$$
\begin{aligned}
& \widehat{\alpha}_{1 l j}^{(\mathbb{A}, t)}=\frac{1}{n} \sum_{r \neq q \in \mathbb{T}_{\mathbb{A}, l, t}} X_{l q} X_{l r} R_{q t}^{(\mathbb{A}, l)} R_{j t}^{(\mathbb{A}, l)} R_{j r}^{(\mathbb{A}, l)}\left(R_{j j}^{(\mathbb{A}, l)} R_{t t}^{(\mathbb{A}, l)}\right)^{-1}, \\
& \widehat{\alpha}_{2 l j}^{(\mathbb{A}, t)}=\frac{1}{n} \sum_{r \neq q \in \mathbb{T}_{\mathbb{A}, l, t}} X_{l q} X_{l r} R_{r t}^{(\mathbb{A}, l)} R_{j t}^{(\mathbb{A}, l)} R_{j q}^{(\mathbb{A}, l, t)}\left(R_{t t}^{(\mathbb{A}, l)} R_{j j}^{(\mathbb{A}, l)}\right)^{-1}, \\
& \widehat{\alpha}_{3 l j}^{(\mathbb{A}, t)}=\frac{1}{n} \sum_{r \neq q \in \mathbb{T}_{\mathbb{A}, l, t}} X_{l q} X_{l r} R_{j q}^{(\mathbb{A}, l, t)} R_{j r}^{(\mathbb{A}, l, t)}\left(R_{j t}^{(\mathbb{A}, l)}\right)^{2}\left(R_{t t}^{(\mathbb{A}, l)} R_{j j}^{(\mathbb{A}, l)} R_{j j}^{(\mathbb{A}, l, t)}\right)^{-1} .
\end{aligned}
$$


Lemma 3.16. Assuming the conditions of Theorem 1.1, there exist constants $C, c$ such that

$$
\begin{aligned}
\mathbf{E}^{\frac{1}{m}}\left\{\left|\alpha_{l j}^{(\mathbb{A}, t)}\right|^{m} \mid \mathfrak{M}^{(\mathbb{A}, j, t)}\right\} \leq & \frac{C m^{3}}{(n v)^{\frac{3}{2}}}\left(v\left(\sum_{q \in \mathbb{T}_{t}}\left|R_{q l}^{(j, t)}\right|^{2}\right) l_{n, \alpha}^{\frac{2}{\varkappa}} \sqrt{\kappa_{n}} \mathbb{I}\left\{\mathcal{U}^{(j, t)}\right\}+\kappa_{n}^{\frac{3}{2}}\right) \\
& +\Phi_{n} .
\end{aligned}
$$

Proof. For the proof of this lemma see Subsection 9.9 of the Appendix.

Corollary 3.17. Assuming conditions of Theorem 1.1, there exist constants $C, c$ such that

$$
\frac{1}{n} \sum_{l \in \mathbb{T}_{j t}} \mathbf{E}^{\frac{1}{m}}\left\{\left|\alpha_{l j}^{(\mathbb{A}, t)}\right|^{m} \mid \mathfrak{M}^{(\mathbb{A}, j, t)}\right\} \leq \frac{C m^{3}}{(n v)^{\frac{3}{2}}} \kappa_{n}^{\frac{3}{2}}+\Phi_{n} .
$$

Proof. To prove the claim it is enough to use the inequality

$$
\frac{1}{n} \sum_{l \in \mathbb{T}_{j t}} \sum_{q \in \mathbb{T}_{t, j}}\left|R_{q l}^{(t, j)}\right|^{2} \leq v^{-1} \operatorname{Im} m_{n}(z)+\frac{C}{n v} .
$$

Similar to Lemma 3.16 we get

Lemma 3.18. Assuming the conditions of Theorem 1.1, there exist constants $C, c$ such that for $0 \leq s \leq c n v / \kappa_{n}$,

$$
\mathbf{E}^{\frac{1}{m}}\left\{\left|\gamma_{l j}^{(\mathbb{A}, t)}\right|^{m} \mid \mathfrak{M}^{(\mathbb{A}, j, t)}\right\} \leq \frac{C}{n \sqrt{n v}}\left(\sum_{q \in \mathbb{T}_{j, l, t}}\left|R_{q l}^{(j, t)}\right|^{2}\right)^{\frac{1}{2}} \mathbb{I}\left\{\mathcal{U}^{(j, t)}\right\} \sqrt{\kappa_{n}}+\Phi_{n} .
$$

Proof. For the proof of this Lemma see Subsection9.10 of the Appendix.

Corollary 3.19. Assuming conditions of Theorem 1.1, there exist constants $C, c$ such that for $0 \leq s \leq c n v /\left(\psi_{0} l_{n, \alpha}^{\frac{2}{\varkappa}}\right)$,

$$
\frac{1}{n} \sum_{l \in \mathbb{T}_{j t}} \mathbf{E}^{\frac{1}{m}}\left\{\left|\gamma_{l j}^{(\mathbb{A}, t)}\right|^{m} \mid \mathfrak{M}^{(\mathbb{A}, j, t)}\right\} \leq \frac{C m^{3}}{(n v)^{\frac{3}{2}}} \kappa_{n}^{\frac{3}{2}}+\Phi_{n} .
$$

Proof. To prove the claim it is enough to use the inequality

$$
\frac{1}{n} \sum_{l \in \mathbb{T}_{j t}}\left(\sum_{q \in \mathbb{T}_{t, j}}\left|R_{q l}^{(t, j)}\right|^{2}\right)^{\frac{1}{2}} \leq v^{-\frac{1}{2}}\left(\operatorname{Im} m_{n}(z)+\frac{C}{n v}\right)^{\frac{1}{2}} .
$$




\section{Bounds for the Laplace transform of $\widehat{\zeta}$}

Let $g(s)=\mathbf{E} \exp \{s \widehat{\zeta}\}$. Let

$$
\mathcal{E}_{1}=\mathcal{E} \cap \mathcal{U}
$$

In this Section we prove the crucial result for the proof of Theorem 1.1.

Proposition 4.1. Assuming the conditions of Theorem 1.1 and

$$
\left.\mathbf{E} \mathbb{\mathcal { E } _ { 1 } ^ { c }}\right\} \leq \exp \left\{-c l_{n, \alpha}\right\},
$$

there exist constants $C_{1}, C_{2}, c>0$ depending on $\varkappa$ and $A$ only such that for $0 \leq s \leq \mathrm{cnv} /\left(\kappa_{n} \log n\right)$,

$$
g(s) \leq C_{1} \exp \left\{\frac{C_{2} s^{2} \kappa_{n}^{2}}{n^{2} v^{2}}\right\} .
$$

Proof. Consider the derivative of $g(s)$ :

$$
g^{\prime}(s)=\mathbf{E} \widehat{\zeta} \exp \{s \widehat{\zeta}\}=\frac{1}{n} \sum_{j=1}^{n} \mathbf{E} \widehat{\zeta}_{j} \exp \{s \widehat{\zeta}\}
$$

We continue with equality (4.4). Let $k=c \log n$, where the constant $c$ will be chosen later.

Step 1. We have

$$
g^{\prime}(s)=\sum_{\nu=0}^{k} A_{\nu}+A_{k+1}
$$

where

$$
\begin{aligned}
A_{\nu} & =\frac{s^{\nu}}{\nu ! n} \sum_{j=1}^{n} \mathbf{E} \widehat{\zeta}_{j}\left(\widehat{\zeta}-\widehat{\zeta}^{(j)}\right)^{\nu} \exp \left\{s \widehat{\zeta}^{(j)}\right\}, \text { for } \nu=0,1 \ldots, k \\
A_{k+1} & =\frac{1}{n} \sum_{j=1}^{n} \mathbf{E} \widehat{\zeta}_{j}\left(\exp \left\{s\left(\widehat{\zeta}-\widehat{\zeta}^{(j)}\right)\right\}-\sum_{\nu=0}^{k} \frac{s^{\nu}}{\nu !}\left(\widehat{\zeta}-\widehat{\zeta}^{(j)}\right)^{\nu}\right) \exp \left\{s \widehat{\zeta}^{(j)}\right\}
\end{aligned}
$$

Note that

$$
\mathbf{E}\left\{\widehat{\zeta}_{j} \mid \mathfrak{M}^{(j)}\right\}=\mathbf{E}\left\{\zeta_{j} \mathbb{I}\{\mathcal{G}\} \mid \mathfrak{M}^{(j)}\right\}=-\mathbf{E}\left\{\zeta_{j} \mathbb{I}\left\{\left(\mathcal{G}^{c}\right)\right\} \mid \mathfrak{M}^{(j)}\right\}
$$

We use that

$$
\mathbb{I}\left\{\mathcal{G}^{c}\right\} \leq \mathbb{I}\left\{\left(\mathcal{G}^{(j)}\right)^{c}\right\}+\mathbb{I}\left\{\left(\mathcal{G}^{c}\right)\right\} \mathbb{I}\left\{\mathcal{G}^{(j)}\right\}
$$


This implies

$$
\begin{aligned}
\left|A_{0}\right| \leq & \frac{1}{n} \sum_{j=1}^{n} \mathbf{E}\left(\mathbf{E}\left\{\left|\zeta_{j}\right| \mid \mathfrak{M}^{(j)}\right\}\right) \mathbb{I}\left\{\left(\mathcal{G}^{(j)}\right)^{c}\right\} \exp \left\{s \widehat{\zeta}^{(j)}\right\} \\
& +\frac{1}{n} \sum_{j=1}^{n} \mathbf{E}\left(\mathbf{E}\left\{\left|\zeta_{j}\right| \mathbb{I}\left\{\left(\mathcal{G}^{c}\right)\right\} \mid \mathfrak{M}^{(j)}\right\}\right) \mathbb{I}\left\{\mathcal{G}^{(j)}\right\} \exp \left\{s \widehat{\zeta}^{(j)}\right\} .
\end{aligned}
$$

Note that

$$
\begin{aligned}
\mathbf{E}\left\{\left(\mathbf{E}\left\{\left|\zeta_{j}\right| \mid \mathfrak{M}^{(j)}\right\}\right) \mathbb{I}\left\{\left(\mathcal{G}^{(j)}\right)^{c}\right\} \exp \left\{s \widehat{\zeta}^{(j)}\right\}\right. & =\mathbf{E}\left|\zeta_{j}\right| \mathbb{I}\left\{\left(\mathcal{G}^{(j)}\right)^{c}\right\} \\
& \leq \mathbf{E}^{\frac{1}{2}}\left|\zeta_{j}\right|^{2} \mathbf{E}^{\frac{1}{2}} \mathbb{I}\left\{\left(\mathcal{G}^{(j)}\right)^{c}\right\} .
\end{aligned}
$$

We use here that $\mathbb{I}\left\{\left(\mathcal{G}^{(j)}\right)^{c}\right\} \exp \left\{\widehat{\zeta}^{(j)}\right\}=\mathbb{I}\left\{\left(\mathcal{G}^{(j)}\right)^{c}\right\}$. Note that, for $v \geq v_{0}$,

$$
\mathbf{E}\left|\zeta_{j}\right|^{2} \leq n^{-1} v^{-1} \mathbf{E} \operatorname{Im} m_{n}^{(j)}(z) \leq C .
$$

The inequalities (4.7) and (4.8) together imply

$$
\mathbf{E}\left(\mathbf{E}\left\{\left|\zeta_{j}\right| \mathbb{I}\left\{\left(\mathcal{G}^{(j)}\right)^{c}\right\} \mid \mathfrak{M}^{(j)}\right\}\right) \exp \left\{s \widehat{\zeta}^{(j)}\right\} \leq \exp \left\{-c l_{n, \alpha}\right\} g(s) .
$$

Furthermore, applying Cauchy's inequality, we get

$$
\mathbf{E}\left\{\left|\zeta_{j}\right| \mathbb{I}\left\{\mathcal{G}^{c}\right\} \mathbb{I}\left\{\mathcal{G}^{(j)}\right\} \mid \mathfrak{M}^{(j)}\right\} \leq \mathbf{E}^{\frac{1}{2}}\left\{\left|\zeta_{j}\right|^{2} \mid \mathfrak{M}^{(j)}\right\} \mathbf{E}^{\frac{1}{2}}\left\{\mathbb{I}\left\{\mathcal{G}^{c}\right\} \mathbb{I}\left\{\mathcal{G}^{(j)}\right\} \mid \mathfrak{M}^{(j)}\right\} .
$$

According to Remark 9.2 we have

$$
\mathbf{E}\left\{\mathbb{I}\left\{\left(\mathcal{G}^{c}\right)\right\} \mathbb{I}\left\{\mathcal{G}^{(j)}\right\} \mid \mathfrak{M}^{(j)}\right\} \leq \exp \left\{-c l_{n, \alpha}\right\}+\Phi_{n} .
$$

Moreover by definition of $\zeta_{j}$ and (2.5)

$$
\mathbf{E}\left\{\left|\zeta_{j}\right|^{2} \mid \mathfrak{M}^{(j)}\right\} \leq \frac{C}{n v} \operatorname{Im} m_{n}^{(j)}(z) .
$$

Inequalities (4.6) - (4.12) together imply

$$
\left|A_{0}\right| \leq \frac{C}{(n v)^{4}} \mathbf{E} \exp \left\{s \widehat{\zeta}^{(j)}\right\} .
$$

For any $\mathbb{A} \subset \mathbb{T}$ and for $j \notin \mathbb{A}$, we have

$$
\widehat{\zeta}^{(\mathbb{A})}-\widehat{\zeta}^{(\mathbb{A}, j)}=\frac{1}{n} \widehat{\zeta}_{j}^{(\mathbb{A})}+\frac{1}{n} \sum_{l \in \mathbb{T}_{\mathbb{A}, j}}\left(\widehat{\zeta}_{l}^{(\mathbb{A})}-\widehat{\zeta}_{l}^{(\mathbb{A}, j)}\right) .
$$


Furthermore,

$\widehat{\zeta}_{l}^{(\mathbb{A})}-\widehat{\zeta}_{l}^{(\mathbb{A}, j)}=\widehat{\zeta}_{l}^{(\mathbb{A})} \mathbb{I}\left\{\left(\mathcal{G}^{\mathbb{A}, j)}\right)^{c}\right\}-\widehat{\zeta}_{l}^{(\mathbb{A}, j)} \mathbb{I}\left\{\left(\mathcal{G}^{\mathbb{A})}\right)^{c}\right\}+\left(\zeta_{l}^{(\mathbb{A})}-\zeta_{l}^{(\mathbb{A}, j)}\right) \mathbb{I}\left\{\mathcal{G}^{\mathbb{A}, j)}\right\} \mathbb{I}\left\{\mathcal{G}^{\mathbb{A})}\right\}$.

Recall that

$$
\begin{aligned}
& \eta_{l j}^{(\mathbb{A})}=\frac{2}{n} \sum_{q \in \mathbb{T}_{\mathbb{A}, l, j}} X_{l q} X_{l j} R_{j q}^{(\mathbb{A}, l)}, \\
& \xi_{l j}^{(\mathbb{A})}=\frac{1}{n} \sum_{q \neq r \in \mathbb{T}_{\mathbb{A}, l, j}} X_{l q} X_{l r} R_{j q}^{\mathbb{A}, l} R_{j r}^{(\mathbb{A}, l)}\left(R_{j j}^{(\mathbb{A}, l)}\right)^{-1}
\end{aligned}
$$

and

$$
\widehat{\eta}_{l j}^{(\mathbb{A})}=\eta_{l j}^{(\mathbb{A})} \mathbb{I}\left\{\mathcal{G}^{(\mathbb{A})}\right\} \mathbb{I}\left\{\mathcal{G}^{(\mathbb{A}, j)}\right\}, \quad \widehat{\xi}_{l j}^{(\mathbb{A})}=\xi_{l j}^{(\mathbb{A})} \mathbb{I}\left\{\mathcal{G}^{(\mathbb{A})}\right\} \mathbb{I}\left\{\mathcal{G}^{(\mathbb{A}, j)}\right\} .
$$

We may write now

$$
\widehat{\zeta}_{l}^{(\mathbb{A})}-\widehat{\zeta}_{l}^{(\mathbb{A}, j)}=r_{l j}^{(\mathbb{A})}+\widehat{\xi}_{l j}^{(\mathbb{A})}+\widehat{\eta}_{l j}^{(\mathbb{A})},
$$

where

$$
r_{l j}^{(\mathbb{A})}=\widehat{\zeta}_{l}^{(\mathbb{A})} \mathbb{I}\left\{\left(\mathcal{G}^{\mathbb{A}, j)}\right)^{c}\right\}-\widehat{\zeta}_{l}^{(\mathbb{A}, j)} \mathbb{I}\left\{\left(\mathcal{G}^{\mathbb{A})}\right)^{c}\right\} .
$$

We represent $\widehat{\zeta}^{(\mathbb{A})}-\widehat{\zeta}^{(\mathbb{A}, j)}$ in the form

$$
\widehat{\zeta}^{(\mathbb{A})}-\widehat{\zeta}^{(\mathbb{A}, j)}=\frac{1}{n} \sum_{l \in \mathbb{T}_{\mathbb{A}}} \theta_{l, j}^{(\mathbb{A})},
$$

where

$$
\theta_{l, j}^{(\mathbb{A})}=\left\{\begin{array}{l}
\widehat{\zeta}_{j}^{(\mathbb{A})}, \text { if } l=j, \\
\widetilde{\xi}_{l j}^{(\mathbb{A})}+\widehat{\eta}_{l j}^{(\mathbb{A})}, \text { otherwise. }
\end{array}\right.
$$

In what follows we shall consider $\mathbb{A}=\emptyset$. Note that by definition

$$
\left|\theta_{l, j}\right| \leq \frac{C \sqrt{\kappa_{n}}}{\sqrt{n v}} \text { a.s. }
$$

Denote by

$$
\sum_{j_{1}, \ldots, j_{t}}^{*}=\sum_{j_{1} \in \mathbb{T}_{j_{0}}} \sum_{j_{2} \in \mathbb{T}_{j_{0}, j_{1}}} \ldots \sum_{j_{t} \in \mathbb{T}_{j_{0}, \ldots, j_{t-1}}} .
$$

Using these notations we may write

$$
\begin{aligned}
\mathbf{E} \widehat{\zeta}_{j_{0}}(\widehat{\zeta} & \left.-\widehat{\zeta}^{\left(j_{0}\right)}\right)^{\nu} \exp \left\{\widehat{\zeta}^{\left(j_{0}\right)}\right\}=\frac{1}{n^{\nu}} \sum_{j_{1}, \ldots, j_{\nu}}^{*} \mathbf{E} \widehat{\zeta}^{\left(j_{0}\right)} \prod_{t=1}^{\nu} \theta_{j_{t}, j_{0}} \exp \left\{\widehat{\zeta}^{\left(j_{0}\right)}\right\} \\
& +\frac{1}{n^{\nu}} \sum_{t=1}^{\nu-1} \sum_{\substack{\mu_{1}, \ldots, \mu_{t} \geq 1 \\
\mu_{1}+\ldots, \mu_{t}=\nu}} \sum_{j_{1}, \ldots, j_{t}}^{*} \mathbf{E} \widehat{\zeta}_{j_{0}} \prod_{l=1}^{t} \theta_{j_{l}, j_{0}}^{\mu_{l}} \exp \left\{\widehat{\zeta}^{\left(j_{0}\right)}\right\}=: V_{1 \nu}+V_{2 \nu}
\end{aligned}
$$


Lemma 4.1. Assuming the conditions of Theorem 1.1 there exist constants $c, C>0$ such that for $0 \leq s \leq c n v /\left(\kappa_{n} \log n\right)$

$$
\left|V_{2 \nu}\right| \leq\left(\frac{C \kappa_{n}}{n v}\right)^{\nu+1} g(s) .
$$

Proof. Applying inequality (4.16) and Hölder's inequality for conditional expectations, we get

$$
\begin{aligned}
\left|\mathbf{E} \widehat{\zeta}_{j_{0}} \prod_{l=1}^{t} \theta_{j_{l}, j_{0}}^{\mu_{l}} \exp \left\{\widehat{\zeta}^{\left(j_{0}\right)}\right\}\right| \leq & \frac{C \sqrt{\kappa_{n}}}{\sqrt{n v}}\left(\frac{C \sqrt{\kappa_{n}}}{\sqrt{n v}}\right)^{\nu-t} \\
& \times \mathbf{E} \prod_{l=1}^{t} \mathbf{E}^{\frac{1}{t+1}}\left\{\left|\theta_{j_{l}, j_{0}}\right|^{t+1} \mid \mathfrak{M}^{\left(j_{0}\right)}\right\} \exp \left\{s \widehat{\zeta}^{\left(j_{0}\right.}\right\}
\end{aligned}
$$

Summing the result in $j_{1}, \ldots, j_{t}$ and applying Lemmas 3.11 and 3.12, we obtain

$$
V_{2 \nu} \leq \frac{C \sqrt{\kappa_{n}}}{\sqrt{n v}} \sum_{t=1}^{\nu-1}\left(\frac{C \sqrt{\kappa_{n}}}{n \sqrt{n v}}\right)^{\nu-t}\left(\begin{array}{c}
\nu-1 \\
t-1
\end{array}\right) \mathbf{E} Q_{j_{0}} \exp \left\{s \widehat{\zeta}^{\left(j_{0}\right)}\right\}
$$

where

$$
\begin{array}{r}
Q_{j_{0}}=\frac{1}{n^{t}} \sum_{j_{1}, \ldots, j_{t}}^{*} \prod_{l=1}^{t}\left(\operatorname { m a x } \left\{\frac{t l_{n, \alpha}^{\frac{2}{\varkappa}}}{n}\left(\left(\sum_{q \in \mathbb{T}_{j_{0}, j_{l}}}\left|R_{j_{l}, q}^{\left(j_{0}\right)}\right|^{2}\right)^{\frac{1}{2}} \mathbb{I}\left\{\mathcal{U}^{\left(j_{0}\right)}\right\}+\psi_{0}\right)+\Phi_{n},\right.\right. \\
\left.\left.\frac{C t l_{n, \alpha}^{\frac{2}{\varkappa}}}{n v}\left(v \sum_{t \in \mathbb{T}_{j_{0}, l}}\left|R_{j_{l}, t}^{\left(j_{0}\right)}\right|^{2} \mathbb{I}\left\{\mathcal{U}^{\left(j_{0}\right)}\right\}+\psi_{0}\right)+\Phi_{n}\right\}\right) .
\end{array}
$$

After summing in $j_{1}, \ldots, j_{t}$ similar to the proof of Lemma 3.14 we get

$$
Q_{j_{0}} \leq\left(\frac{t \kappa_{n}}{n v}+\Phi_{n}\right)^{t}
$$

This inequality implies

$$
\mathbf{E} Q_{j_{0}} \exp \left\{s \widehat{\zeta}^{\left(j_{0}\right)}\right\} \leq 2^{t} \mathbf{E}\left(\left(\kappa_{n}\right)^{t}+\Phi_{n}\right) \exp \left\{s \widehat{\zeta}^{\left(j_{0}\right)}\right\}
$$

For $s \leq c^{\prime} n v /\left(\varkappa_{n} \log n\right)$, we get, for some constants $c^{\prime}, C>0$

$$
\mathbf{E} Q_{j_{0}} \exp \left\{s \widehat{\zeta}^{\left(j_{0}\right)}\right\} \leq C^{t} \kappa_{n}^{t} g(s) .
$$


Bounding now the sum on the right hand side of (4.20) we get

$$
\begin{aligned}
V_{2, \nu} & \leq\left(\frac{C \nu \kappa_{n}}{n v}\right)^{\nu+1} \sqrt{\frac{v}{\psi_{0}}} \sum_{t=0}^{\nu-1}\left(\begin{array}{c}
\nu-1 \\
t
\end{array}\right)\left(\frac{C v}{n \psi_{0}}\right)^{\frac{\nu-t-1}{2}} g(s) \\
& \leq\left(\frac{C \kappa_{n} \log n}{n v}\right)^{\nu+1} \sum_{t=0}^{\nu-1}\left(\begin{array}{c}
\nu-1 \\
t
\end{array}\right)\left(\frac{C \sqrt{v}}{n}\right)^{\frac{\nu-t-1}{2}} g(s) \leq\left(\frac{C \kappa_{n} \log n}{n v}\right)^{\nu+1} g(s) .
\end{aligned}
$$

Thus Lemma 4.1 is proved.

Corollary 4.2. Assuming the conditions of Theorem 1.1, there exist constants $c, C>0$ such that, for $0 \leq s \leq \mathrm{cnv} /\left(\kappa_{n} \log n\right)$,

$$
\sum_{\nu=1}^{k+1} \frac{s^{\nu}}{\nu !}\left|V_{2 \nu}\right| \leq\left(\frac{C s \kappa_{n}^{2} \log n}{(n v)^{2}}\right) g(s) .
$$

Proof. Note that we may chose a constant $c>0$ depending on $C>0$ defined in Lemma 4.1 such that

$$
\frac{C s \kappa_{n} \log n}{n v} \leq C_{0}<1
$$

We have

$$
\sum_{\nu=1}^{k} \frac{s^{\nu}}{\nu !}\left|V_{2 \nu}\right| \leq \frac{C s \kappa_{n}^{2} \log n}{(n v)^{2}} \sum_{\nu=0}^{k} \frac{1}{\nu !} C_{0}^{\nu} g(s) \leq \frac{C s \kappa_{n}^{2} \log n}{(n v)^{2}}
$$

Thus Corollary 4.2 is proved.

Lemma 4.3. Assuming the conditions of Theorem 1.1 there exist constants $c, C>0$ such that for $0 \leq s \leq c n v /\left(\psi_{0} l_{n, \alpha}^{\frac{2}{\varkappa}}\right)$

$$
\left|A_{k+1}\right| \leq\left(\frac{s C l_{n, \alpha}^{\frac{2}{\varkappa}} \psi_{0}^{2}}{(n v)^{2}(k+1) !}\right) g(s)
$$

Proof. First we note

$$
\left|A_{k+1}\right| \leq \frac{s^{k+1}}{(k+1) !} \mathbf{E}\left|\widehat{\zeta}_{j_{0}}\right|\left|\widehat{\zeta}-\widehat{\zeta}^{\left(j_{0}\right.}\right|^{k+1} \exp \left\{s \widehat{\zeta}^{\left(j_{0}\right)}\right\}
$$


Repeating the arguments of inequalities (4.19) - (4.23), we obtain

$$
\left|A_{k+1}\right| \leq \frac{s}{(k+1) !} \frac{C l_{n, \alpha}^{\frac{2}{\varkappa}} \sqrt{\psi_{0}}}{\sqrt{n v}}\left(\frac{C s l_{n, \alpha}^{\frac{2}{\varkappa}} \psi_{0}}{n v}\right)^{k} g(s) .
$$

Choosing $k=c \log n$ and $s$ such that

$$
\frac{C s l_{n, \alpha}^{\frac{2}{\varkappa}}}{n v} \leq \alpha<1
$$

we get

$$
\left|A_{k+1}\right| \leq \frac{s l_{n, \alpha}^{\frac{2}{2}} \psi_{0}}{(n v)^{2}(k+1) !} g(s) .
$$

Thus Lemma 4.3 is proved.

Inequalities (4.13), (4.24) and (4.25) together imply, for $0 \leq s \leq c n v / \kappa_{n}$

$$
g^{\prime}(s) \leq C\left(\frac{1}{n^{2} v^{2}}+\frac{C s l_{n, \alpha}^{\frac{2}{\varkappa}} \psi_{0}^{2}}{(n v)^{2}}\right) g(s)+\frac{1}{n} \sum_{j_{0}=1}^{n} \sum_{\nu=1}^{k} \frac{s^{\nu}}{\nu !} V_{1, \nu}
$$

Step 2. We consider now the quantities $V_{1, \nu_{1}}$ for $\nu_{1}=1, \ldots, k$,

$$
V_{1, \nu_{1}}=\frac{1}{n^{\nu_{1}}} \sum_{j_{1}, \ldots, j_{\nu_{1}}}^{*} \mathbf{E} \widehat{\zeta}_{j_{0}} \prod_{t=1}^{\nu_{1}} \theta_{j_{t}, j_{0}} \exp \left\{\widehat{\zeta}^{\left(j_{0}\right)}\right\}
$$

We represent $V_{1, \nu_{1}}$ in the form

$$
V_{1, \nu_{1}}=W_{1, \nu_{1}}+W_{2, \nu_{1}}
$$

where

$$
\begin{aligned}
W_{1, \nu_{1}} & =\frac{1}{n^{\nu_{1}}} \sum_{j_{1}, \ldots, j_{\nu_{1}}}^{*} \mathbf{E} \widehat{\zeta}_{j_{0}} \prod_{t=1}^{\nu_{1}} \theta_{j_{t}, j_{0}} \exp \left\{\widehat{\zeta}^{\left(j_{0}, j_{\nu_{1}}\right)}\right\} \\
W_{2, \nu_{1}} & =\frac{1}{n^{\nu}} \sum_{j_{1}, \ldots, j_{\nu_{1}}}^{*} \mathbf{E} \widehat{\zeta}_{j_{0}} \prod_{t=1}^{\nu_{1}} \theta_{j_{t}, j_{0}} P_{j_{0}, j_{\nu_{1}}} \exp \left\{\widehat{\zeta}^{\left(j_{0}, j_{\nu_{1}}\right)}\right\}
\end{aligned}
$$

with

$$
P_{j_{0}, j_{\nu_{1}}}=\left(\exp \left\{s\left(\widehat{\zeta}^{\left(j_{0}\right)}-\widehat{\zeta}^{\left(j_{0}, j_{\nu_{1}}\right)}\right)\right\}-1\right)
$$


We bound $W_{2, \nu_{1}}$ repeating the arguments of Step 1. First we represent $W_{2, \nu_{1}}$ in the form

$$
W_{2 \nu_{1}}=\sum_{\nu_{2}=1}^{k-\nu_{1}} \frac{s^{\nu_{2}}}{\nu_{2} !} W_{2, \nu_{1}, \nu_{2}}+W_{2, \nu_{1}, k-\nu_{1}+1}
$$

where

$$
\begin{aligned}
& W_{2, \nu_{1}, \nu_{2}}= \frac{1}{n^{\nu_{1}}} \sum_{j_{1}, \ldots, j_{\nu_{1}}}^{*} \mathbf{E} \widehat{\zeta}_{j_{0}} \prod_{t=1}^{\nu_{1}} \theta_{j_{t}, j_{0}}\left(\widehat{\zeta}^{\left(j_{0}\right)}-\widehat{\zeta}^{\left(j_{0}, j_{\nu_{1}}\right)}\right)^{\nu_{2}} \exp \left\{\widehat{\zeta}^{\left(j_{0}, j_{\nu_{1}}\right)}\right\} \\
& W_{2, \nu_{1}, k-\nu_{1}+1}=\frac{1}{n^{\nu_{1}}} \sum_{j_{1}, \ldots, j_{\nu_{1}}}^{*} \mathbf{E} \widehat{\zeta}_{j_{0}} \prod_{t=1}^{\nu_{1}} \theta_{j_{t}, j_{0}}\left(\exp \left\{s\left(\widehat{\zeta}^{\left(j_{0}\right)}-\widehat{\zeta}^{\left(j_{0}, j_{\nu_{1}}\right)}\right)\right\}\right. \\
& \\
&\left.-\sum_{\nu_{2}=0}^{k-\nu_{1}} \frac{s^{\nu_{2}}}{\nu_{2} !}\left(\widehat{\zeta}^{\left(j_{0}\right)}-\widehat{\zeta}^{\left(j_{0}, j_{\nu_{1}}\right)}\right)^{\nu_{2}}\right) \exp \left\{\widehat{\zeta}^{\left(j_{0}, j_{\nu_{1}}\right)}\right\}
\end{aligned}
$$

Similar to formula (4.18) we represent

$$
W_{2, \nu_{1}, \nu_{2}}=V_{1, \nu_{1}, \nu_{2}}+V_{2, \nu_{1}, \nu_{2}},
$$

where

$$
\begin{aligned}
& V_{1, \nu_{1}, \nu_{2}}=\frac{1}{n^{\nu_{1}}} \sum_{j_{1}^{(1)}, \ldots, j_{\nu_{1}}^{(1)}}^{*} \frac{1}{n^{\nu_{2}}} \sum_{j_{1}^{(2)}, \ldots, j_{\nu_{2}}^{(2)}}^{* *} \mathbf{E} \widehat{\zeta}_{j_{0}} \prod_{t=1}^{\nu_{1}} \theta_{j_{t}^{(1)}, j_{0}} \times \prod_{t=1}^{\nu_{2}} \theta_{j_{t}^{(2)}, j_{\nu_{1}}^{(1)}} \exp \left\{\widehat{\zeta}^{\left(j_{0}, j_{\nu_{1}}^{(1)}\right)}\right\} \\
& V_{2, \nu_{1}, \nu_{2}}=\frac{1}{n^{\nu_{1}}} \frac{1}{n^{\nu_{2}}} \sum_{j_{1}^{(1)}, \ldots, j_{\nu_{1}}^{(1)}}^{*} \sum_{t=1}^{\nu_{2}^{(2)}-1} \sum_{\substack{\mu_{1}, \ldots, \mu_{t} \geq 1: \\
\mu_{1}+\cdots+\mu_{t}=\nu_{2}^{(2)}}} \\
& \times \sum_{j_{1}^{(2)}, \ldots, j_{\nu_{2}}^{(2)}}^{*} \mathbf{E} \widehat{\zeta}_{j_{0}} \prod_{l=1}^{\nu_{1}} \theta_{j_{l}^{(1)}, j_{0}} \prod_{l=1}^{t} \theta_{j_{l}^{(2)}, j_{\nu_{1}}^{(1)}}^{\mu_{l}} \exp \left\{\widehat{\zeta}^{\left(j_{0}\right)}, j_{\nu_{1}}^{(1)}\right\},
\end{aligned}
$$

where

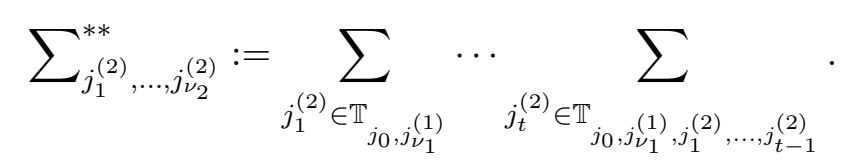


Similarly to Corollary 4.2 we may prove

$$
\sum_{\nu_{1}=1}^{k} \sum_{\nu_{2}=1}^{k-\nu_{1}+1} \frac{s^{\nu_{1}}}{\nu !} \frac{s^{\nu_{2}}}{\nu_{2} !}\left|V_{2, \nu_{1}, \nu_{2}}\right| \leq\left(\frac{C s l_{n, \alpha}^{\frac{2}{2}} \psi_{0}^{2}}{(n v)^{2}}\right) g(s) .
$$

Analogously to inequality (4.25), we get

$$
\left|W_{2, \nu_{1}, k-\nu_{1}+1}\right| \leq \frac{s}{\nu_{1} !\left(k-\nu_{1}+1\right) !} \frac{C l_{n, \alpha}^{\frac{2}{\varkappa}}}{\sqrt{n v}}\left(\frac{C s l_{n, \alpha}^{\frac{2}{\varkappa}} \psi_{0}^{2}}{n v}\right)^{k} g(s) .
$$

Consider now the bound for $W_{1, \nu_{1}}$. We introduce the notation

$$
\Delta \zeta_{j_{0}, j_{\nu_{1}}}=\widehat{\zeta}_{j_{0}}-\widehat{\zeta}_{j_{0}}^{\left(j_{\nu_{1}}\right)}, \quad \Delta \theta_{j_{t}, j_{0}, j_{\nu_{1}}}=\theta_{j_{t}, j_{0}}-\theta_{j_{t}, j_{0}}^{\left(j_{\nu_{1}}\right)}
$$

We may represent

$$
W_{1, \nu_{1}}=\frac{1}{n^{\nu_{1}}} \sum_{j_{1}, \ldots, j_{\nu}}^{*} \mathbf{E} \widehat{\zeta}_{j_{0}} \prod_{t=1}^{\nu_{1}-1} \theta_{j_{t}, j_{0}}^{\left(j_{\nu_{1}}\right)} \theta_{j_{0}, j_{\nu_{1}}} \exp \left\{\widehat{\zeta}^{\left(j_{0}, j_{\nu_{1}}\right)}\right\}+\sum_{l=0}^{\nu_{1}-1} \widehat{W}_{1, \nu_{1}, l}
$$

where

$$
\widehat{W}_{1, \nu_{1}, 0}=\frac{1}{n^{\nu_{1}}} \sum_{j_{1}, \ldots, j_{\nu}}^{*} \mathbf{E} \Delta \zeta_{j_{0}, j_{\nu_{1}}} \times \prod_{t=1}^{\nu_{1}} \theta_{j_{t}, j_{0}} \exp \left\{\widehat{\zeta}^{\left(j_{0}, j_{\nu_{1}}\right)}\right\}
$$

and, for $l=1, \ldots, \nu_{1}-1$

$$
\widehat{W}_{1, \nu_{1}, l}=\frac{1}{n^{\nu_{1}}} \sum_{j_{1}, \ldots, j_{\nu}}^{*} \mathbf{E} \widehat{\zeta}_{j_{0}}^{\left(j_{\nu_{1}}\right)} \prod_{t=1}^{l-1} \theta_{j_{t}, j_{0}}^{\left(j_{\nu_{1}}\right)} \Delta \zeta_{j_{l}, j_{\nu_{1}}} \prod_{t=l+1}^{\nu_{1}} \theta_{j_{t}, j_{0}} \exp \left\{\widehat{\zeta}^{\left(j_{0}, j_{\nu_{1}}\right)}\right\} .
$$

Lemma 4.4. Assuming the conditions of Theorem 1.1, there exist constants $C, c>0$ such that for any $0 \leq s \leq c n v / \kappa_{n}$, for $l=0, \ldots, \nu_{1}-1$,

$$
\sum_{\nu_{1}=1}^{k} \frac{s^{\nu_{1}}}{\nu_{1} !} \sum_{l=0}^{\nu_{1}-1}\left|\widehat{W}_{1, \nu_{1}, l}\right| \leq \frac{C l_{n, \alpha}^{\frac{2}{\varkappa}} s \psi_{0}^{2}}{(n v)^{2}} g(s) .
$$

We consider first $\widehat{W}_{1, \nu_{1}, 0}$. Applying Hölder's inequality for conditional expectations, we get

$$
\begin{aligned}
\left|\widehat{W}_{1, \nu_{1}, 0}\right| \leq \frac{1}{n^{\nu_{1}}} \sum_{j_{1}, \ldots, j_{\nu}}^{*} \mathbf{E} & \left\{\mathbf{E}^{\frac{1}{\nu_{1}+1}}\left\{\left|\Delta \zeta_{j_{0}, j_{\nu_{1}}}\right|^{\nu_{1}+1} \mid \mathfrak{M}^{\left(j_{0}, j_{\nu_{1}}\right)}\right\}\right. \\
& \left.\times \prod_{t=1}^{\nu_{1}} \mathbf{E}^{\frac{1}{\nu_{1}+1}}\left\{\left|\theta_{j_{t}, j_{0}}\right|^{\nu_{1}+1} \mid \mathfrak{M}^{\left(j_{0}, j_{\nu_{1}}\right)}\right\} \exp \left\{\widehat{\zeta}^{\left(j_{0}, j_{\nu_{1}}\right)}\right\}\right\} .
\end{aligned}
$$


Similar to inequality (4.20) using the results of Lemmas 3.14 and 3.16, we get

$$
\left|\widehat{W}_{1, \nu_{1}, 0}\right| \leq \frac{C s l_{n, \alpha}^{\frac{2}{\varkappa}} \psi_{0}^{2}}{n^{2} v^{2}} g(s) .
$$

Analogously we may represent $V_{1, \nu_{1}, \nu_{2}}$ in the form

$$
\begin{aligned}
V_{1, \nu_{1}, \nu_{2}}=\frac{1}{n^{\nu_{1}}} \frac{1}{n^{\nu_{2}}} \sum_{j_{1}^{(1)}, \ldots, j_{\nu_{1}}^{(1)}}^{*} \sum_{j_{1}^{(2)}, \ldots, j_{\nu_{2}}(2)}^{* *} \mathbf{E} \widehat{\zeta}_{j_{0}} \prod_{t=1}^{\nu_{1}} \theta_{j_{t}^{(1)}, j_{0}} \prod_{t=1}^{\nu_{2}} \theta_{j_{t}^{(2)}, j_{\nu_{1}}^{(1)}} \\
\times \exp \left\{\widehat{\zeta}^{\left(j_{0}, j_{\nu_{1}}, j_{\nu_{2}}\right)}\right\}+Q
\end{aligned}
$$

where

$$
\begin{aligned}
Q=\frac{1}{n^{\nu_{1}}} \frac{1}{n^{\nu_{2}}} \sum_{j_{1}^{(1)}, \ldots, j_{\nu_{1}}^{(1)}}^{*} \sum_{j_{1}^{(2)}, \ldots, j_{\nu_{2}}(2)}^{* *} \mathbf{E} \widehat{\zeta}_{j_{0}} \prod_{t=1}^{\nu_{1}} \theta_{j_{t}^{(1)}, j_{0}} \prod_{t=1}^{\nu_{2}} \theta_{j_{t}^{(2)}, j_{\nu_{1}}^{(1)}} \\
\times\left(\exp \left\{\widehat{\zeta}^{\left(j_{0}, j_{\nu_{1}}\right)}\right\}-\exp \left\{\widehat{\zeta}^{\left(j_{0}, j_{\nu_{1}}, j_{\nu_{2}}\right)}\right\}\right) .
\end{aligned}
$$

We shall again continue to expand $Q$ until we get $k$ factors in the expectation. This leads to the inequality

$$
g^{\prime}(s) \leq\left(\frac{C s \kappa_{n}^{2}}{n^{2} v^{2}}+\frac{\psi_{0}^{2}}{n^{2} v^{2}}\right) g(s),
$$

for $0 \leq s \leq \mathrm{cnv} /\left(\kappa_{n} \log n\right)$ with some positive constant $c>0$. It implies that

$$
g(s) \leq C \exp \left\{\frac{C s^{2} \kappa_{n}^{2}}{n^{2} v^{2}}\right\}
$$

for $0 \leq s \leq c n v /\left(\kappa_{n} \log n\right)$.

Thus Proposition 4.1 is proved.

\section{$5 \quad$ Large deviations}

In this Section we bound

$$
\delta_{n}=\frac{1}{n} \sum_{j=1}^{n} \varepsilon_{j}
$$

We denote by

$$
\delta_{n i}=\frac{1}{n} \sum_{j=1}^{n} \varepsilon_{j i}, \text { for } i=1, \ldots, 4,
$$

and start from $\delta_{n 1}$. 
Lemma 5.1. There exist constants $c$ and $C$ depending on $\varkappa$ and $\alpha$ such that

$$
\operatorname{Pr}\left\{\left|\delta_{n 1}\right|>n^{-1} \beta_{n}\right\} \leq C \exp \left\{-c l_{n, \alpha}\right\} .
$$

Proof. Recall that

$$
\delta_{n 1}=\frac{1}{n \sqrt{n}} \sum_{j=1}^{n} X_{j j} .
$$

By Remark 3.1. $\left|X_{j j}\right| \leq C l_{n, \alpha}^{\frac{1}{\varkappa}}$. Applying McDiarmid's inequality, we get

$$
\operatorname{Pr}\left\{\left|\delta_{n 1}\right|>n^{-1} \beta_{n}\right\} \leq \exp \left\{-c l_{n, \alpha}\right\} .
$$

Thus, Lemma 5.1 is proved.

Corollary 5.2. The following inequality holds

$$
\operatorname{Pr}\left\{\left|\delta_{n 1}\right|>\frac{\beta_{n} \psi_{0}}{n v}\right\} \leq \exp \left\{-c l_{n, \alpha}\right\}
$$

Proof. The proof follows from the inequality $\psi_{0} \geq c \sqrt{v}$ and Lemma 5.1.

Consider now the quantity

$$
\delta_{n 2}:=\frac{1}{n^{2}} \sum_{j=1}^{n} \sum_{l \in \mathbb{T}_{j}}\left(X_{j l}^{2}-1\right) R_{l l}^{(j)} .
$$

We prove the following Lemma

Lemma 5.3. Let $v_{0}=\frac{c_{0} \beta_{n}^{4}}{n}$ with some numerical constant $c_{0} \geq 1$. Under the conditions of Theorem 1.1 there exist constants $c$ and $C$, depending on $\varkappa$ and $\alpha$ only, such that, for $v \geq v_{0}$,

$$
\operatorname{Pr}\left\{\left|\delta_{n 2}\right|>\frac{C l_{n, \alpha}^{\frac{2}{2}}\left(\psi_{0}+\operatorname{Im} m_{n}(z)\right)}{n v}\right\} \leq C \exp \left\{-c l_{n, \alpha}\right\} .
$$

Proof. Introduce the random variables $\xi_{j l}=X_{j l}^{2}-1$, where $\left|X_{j l}\right| \leq l_{n, \alpha}^{\frac{1}{\varkappa}}$. We shall bound the following quantity

$$
\delta_{n 2}=\frac{1}{n^{2}} \sum_{j=1}^{n} \sum_{l \in \mathbb{T}_{j}} \xi_{j l} R_{l l}^{(j)} .
$$


We may represent

$$
\delta_{n 2}=\widehat{\delta}_{n 2}+\widetilde{\delta}_{n 2}+\bar{\delta}_{n 2},
$$

where

$$
\begin{aligned}
& \widehat{\delta}_{n 2}=-\frac{1}{n^{2}} \sum_{j=1}^{n} \sum_{l \in \mathbb{T}_{j}} \xi_{j l} \frac{1}{z+m_{n}(z)}, \\
& \widetilde{\delta}_{n 2}=\frac{1}{n^{2}} \sum_{j=1}^{n} \sum_{l \in \mathbb{T}_{j}} \xi_{j l}\left(R_{l l}^{(j)}+\frac{1}{z+m_{n}^{(j)}(z)}\right), \\
& \bar{\delta}_{n 2}=\frac{1}{n^{2}} \sum_{j=1}^{n} \sum_{l \in \mathbb{T}_{j}} \xi_{j l} \frac{m_{n}^{(j)}(z)-m_{n}(z)}{\left(z+m_{n}(z)\right)\left(z+m_{n}^{(j)}(z)\right)} .
\end{aligned}
$$

First we note

$$
\begin{aligned}
\operatorname{Pr}\left\{\left|\widehat{\delta}_{n 2}\right| \geq \frac{C \beta_{n}}{n}\right\} & \leq \operatorname{Pr}\left\{\left|\widehat{\delta}_{n 2}\right| \geq \frac{C \beta_{n}}{n v},\left|m_{n}(z)-s(z)\right| \leq \frac{1}{2}\right\} \\
& +\operatorname{Pr}\left\{\left|\widehat{\delta}_{n 2}\right| \geq \frac{C l_{n, \alpha}^{\frac{1}{\varkappa}}}{n},\left|m_{n}(z)-s(z)\right|>\frac{1}{2}\right\} \\
& \leq \operatorname{Pr}\left\{\left|\frac{1}{n^{2}} \sum_{j=1}^{n} \sum_{l \in \mathbb{T}_{j}} \xi_{j l}\right| \geq \frac{C^{\prime} l_{n, \alpha}^{\frac{1}{\varkappa}}}{n}\right\} \\
& +\operatorname{Pr}\left\{\left|m_{n}(z)-s(z)\right|>\frac{1}{2}\right\} .
\end{aligned}
$$

The first term we may bound using McDiarmid's inequality and the second one may be bounded using Corollary 3.7. Thus we get

$$
\operatorname{Pr}\left\{\left|\widehat{\delta}_{n 2}\right| \geq \frac{C \beta_{n}}{n}\right\} \leq \exp \left\{-c \sqrt{n v} / l_{n, \alpha}\right\} .
$$

By (3.3), we have

$$
\left|R_{l l}^{(j)}+\frac{1}{z+m^{(j)}(z)}\right| \leq\left|\varepsilon_{l}^{(j)}\right|\left|R_{l l}^{(j)}\right| .
$$

Furthermore, we have

$$
\varepsilon_{l 2}^{(j)}=\sum_{q \in \mathbb{T} j, l}\left(X_{q l}^{2}-1\right) R_{q q}^{(j, l)} .
$$


Conditioning on $\mathfrak{M}^{(j, l)}$ and applying McDiarmid's inequality, we get

$$
\operatorname{Pr}\left\{\left|\varepsilon_{l 2}^{(j)}\right|>\frac{C l_{n, \alpha}^{\frac{2}{\varkappa}}}{\sqrt{n}}, \mathcal{B}^{(j)}\right\} \leq \exp \left\{-c l_{n, \alpha}\right\} .
$$

Using the result of Corollary 3.5 and inequality $\psi_{0} \geq c \sqrt{v}$, we arrive at

$$
\operatorname{Pr}\left\{\left|\varepsilon_{l 2}^{(j)}\right|>\frac{C l_{n, \alpha}^{\frac{2}{\varkappa}} \sqrt{\psi_{0}}}{\sqrt{n v}}, \mathcal{B}^{(j)}\right\} \leq \exp \left\{-c l_{n, \alpha}\right\}
$$

Consider now

$$
\varepsilon_{l 3}^{(j)}=\frac{1}{n} \sum_{q \neq r \in \mathbb{T}_{j, l}} X_{q l} X_{r l} R_{q r}^{(l, j)} .
$$

Representing this quantity as a martingale similar to (9.62) and repeating the arguments (9.63) - (9.66) with $c \sqrt{\log n}$ instead of $\sqrt{n v_{k}}$ in the inequality (9.64) and $x=\gamma_{n} \sqrt{\log n}$ instead $x=\gamma_{n} \sqrt{n v}$, we get

$$
\operatorname{Pr}\left\{\left|\varepsilon_{l 3}^{(j)}\right|>\frac{C \beta_{n}}{\sqrt{n v}}\left(\operatorname{Im} m_{n}^{(j)}(z)\right)^{\frac{1}{2}}\right\} \leq \exp \left\{-c l_{n, \alpha}\right\} .
$$

Furthermore, note that

$$
\left|\varepsilon_{l 1}^{(j)}\right| \leq \frac{C l_{n, \alpha}}{\sqrt{n}} \leq \frac{C l_{n, \alpha} \sqrt{\psi_{0}}}{\sqrt{n v}} \leq C \sqrt{\frac{\kappa_{n}}{n v}} \text { a. s. }
$$

and

$$
\left|\varepsilon_{l 4}^{(j)}\right| \leq \frac{1}{n v} \leq C \sqrt{\frac{\kappa_{n}}{n v}} \text { a.s. }
$$

Inequalities (5.5) - (5.11) together imply

$$
\operatorname{Pr}\left\{\left|\varepsilon_{l}^{(j)}\right| \geq \frac{C l_{n, \alpha}^{\frac{2}{\varkappa}}\left(\sqrt{\operatorname{Im} m_{n}^{(j)}}+\sqrt{\psi_{0}}\right)}{\sqrt{n v}}\right\} \leq \exp \left\{-c l_{n, \alpha}\right\} .
$$

Applying now McDiarmid's inequality, we get

$$
\operatorname{Pr}\left\{\left|\widetilde{\delta}_{n 2}\right| \geq \frac{C l_{n, \alpha}^{\frac{2}{\varkappa}}\left(\sqrt{\operatorname{Im} m_{n}(z)}+\sqrt{\psi_{0}}\right)}{\sqrt{n} \sqrt{n v}}\right\} \leq \exp \left\{-c l_{n, \alpha}\right\} .
$$

Taking into account that $\psi_{0} \geq \sqrt{v}$, we get

$$
\operatorname{Pr}\left\{\left|\widetilde{\delta}_{n 2}\right| \geq \frac{C l_{n, \alpha}^{\frac{2}{\varkappa}}\left(\sqrt{\operatorname{Im} m_{n}(z)}+\sqrt{\psi_{0}}\right) \sqrt{\psi_{0}}}{\sqrt{n} \sqrt{n v}}\right\} \leq \exp \left\{-c l_{n, \alpha}\right\} .
$$


From here, using that $\frac{1}{\sqrt{n}}=\frac{\sqrt{v}}{\sqrt{n v}} \leq \psi_{0}$ and that $\sqrt{\psi_{0} \operatorname{Im} m_{n}(z)} \leq \frac{1}{2}\left(\psi_{0}+\right.$ $\left.\operatorname{Im} m_{n}(z)\right)$, we get

$$
\operatorname{Pr}\left\{\left|\widetilde{\delta}_{n 2}\right| \geq \frac{C l_{n, \alpha}^{\frac{2}{\varkappa}}\left(\operatorname{Im} m_{n}(z)+\psi_{0}\right)}{\sqrt{n} \sqrt{n v}}\right\} \leq \exp \left\{-c l_{n, \alpha}\right\} .
$$

Note that

$$
\left|m_{n}(z)-m_{n}^{(j)}(z)\right| \leq \frac{1}{n v}
$$

This yields that

$$
\operatorname{Pr}\left\{\left|\bar{\delta}_{n 2}\right| \geq \frac{C \beta_{n}^{2}}{n^{\frac{3}{2}} v}\right\} \leq \sum_{j \in \mathbb{T}} \operatorname{Pr}\left\{\frac{1}{n}\left|\sum_{l \in \mathbb{T}_{j}} \xi_{l j}\right| \geq \frac{C \beta_{n}^{2}}{\sqrt{n}}\right\} \leq \exp \{-\ln a\} .
$$

From here, using that $\frac{1}{\sqrt{n}} \leq \frac{\sqrt{v}}{\sqrt{n v}} \leq \psi_{0}$ and that $\sqrt{\psi_{0} \operatorname{Im} m_{n}(z)} \leq \frac{1}{2}\left(\psi_{0}+\right.$ $\left.\operatorname{Im} m_{n}(z)\right)$, we get

$$
\operatorname{Pr}\left\{\left|\bar{\delta}_{n 2}\right| \geq \frac{C \beta_{n}^{2} \psi_{0}}{n v}\right\} \leq \exp \{-\ln a\} .
$$

Thus Lemma 5.3 is proved.

Let now

$$
\delta_{n 3}:=\frac{1}{n^{2}} \sum_{j=1}^{n} \sum_{q \neq r \in \mathbb{T}_{j}} X_{j q} X_{j r} R_{q r}^{(j)}=\frac{1}{n} \sum_{j=1}^{n} \zeta_{j} .
$$

Lemma 5.4. Let $v_{0}=\frac{c_{0} \beta_{n}^{4}}{n}$ with some numerical constant $c_{0}>1$. Assuming the conditions of Theorem 1.1 there exist constants $c$ and $C$, depending on $\varkappa, \alpha$ only such that, for $v \geq v_{0}$,

$$
\operatorname{Pr}\left\{\left|\delta_{n 3}\right|>\frac{4 \beta_{n}^{4} \psi_{0}}{n v}\right\} \leq C \exp \left\{-c l_{n, \alpha}\right\} .
$$

Proof. By Lemma 3.10 ,

$$
\operatorname{Pr}\left\{\delta_{n 3} \neq \frac{1}{n} \sum_{j=1}^{n} \widehat{\zeta}_{j}\right\} \leq \exp \left\{-c l_{n, \alpha}\right\} .
$$

Applying Chebyshev's inequality, we obtain

$$
\operatorname{Pr}\left\{\left|\frac{1}{n} \sum_{j=1}^{n} \widehat{\zeta}_{j}\right|>x\right\} \leq \exp \{-s x\} \mathbf{E} \exp \left\{s \frac{1}{n} \sum_{j=1}^{n} \widehat{\zeta}_{j}\right\}=\exp \{-s x\} g(s) .
$$


We choose $s=c n v /\left(\kappa_{n} \log n\right)$ and $x=\frac{c \kappa_{n} \log ^{4} n}{n v}$. Then, by Proposition 4.1 , we get

$$
\operatorname{Pr}\left\{\left|\frac{1}{n} \sum_{j=1}^{n} \widehat{\zeta}_{j}\right|>x\right\} \leq \exp \left\{-c l_{n, \alpha}\right\} .
$$

Thus the Lemma is proved.

Finally, we shall bound

$$
\delta_{n 4}:=\frac{1}{n^{2}} \sum_{j=1}^{n}\left(\operatorname{Tr} \mathbf{R}-\operatorname{Tr} \mathbf{R}^{(j)}\right) R_{j j} .
$$

Lemma 5.5. For any $z=u+i v$ with $v>0$ the following inequality

$$
\left|\delta_{n 4}\right| \leq \frac{1}{n v} \operatorname{Im} m_{n}(z) \text { a. } s .
$$

holds.

Proof. By formula (5.4) in [11], we have

$$
\left(\operatorname{Tr} \mathbf{R}-\operatorname{Tr} \mathbf{R}^{(j)}\right) R_{j j}=\left(1+\frac{1}{n} \sum_{l, k \in T_{j}} X_{j l} X_{j k}\left(R^{(j)}\right)_{l k}^{2}\right) R_{j j}^{2}=\frac{d}{d z} R_{j j} .
$$

From here it follows that

$$
\frac{1}{n^{2}} \sum_{j=1}^{n}\left(\operatorname{Tr} \mathbf{R}-\operatorname{Tr} \mathbf{R}^{(j)}\right) R_{j j}=\frac{1}{n^{2}} \frac{d}{d z} \operatorname{Tr} \mathbf{R}=\frac{1}{n^{2}} \operatorname{Tr} \mathbf{R}^{2} .
$$

Finally, we note using (2.5) that

$$
\left|\frac{1}{n^{2}} \operatorname{Tr} \mathbf{R}^{2}\right| \leq \frac{1}{n v} \operatorname{Im} m_{n}(z) .
$$

The last inequality concludes the proof. Thus, Lemma 5.5 is proved.

\section{Stieltjes transforms}

We shall derive auxiliary bounds for the difference between the Stieltjes transforms $m_{n}(z)$ of the empirical spectral measure of the matrix $\mathbf{X}$ and 
the Stieltjes transform $s(z)$ of the semi-circular law. Recalling the definitions of $\varepsilon_{j}, \varepsilon_{j \mu}$ in (3.4) and of $\delta_{n \nu}$ in (5.1), (5.2), (5.19) as well as (5.21), we introduce the additional notations

$$
\delta_{n}^{\prime}:=\delta_{n 1}+\delta_{n 2}+\delta_{n 3}, \quad \widehat{\delta}_{n}:=\delta_{n 4}, \quad \bar{\delta}_{n}:=\frac{1}{n} \sum_{\nu=1}^{3} \sum_{j=1}^{n} \varepsilon_{j \nu} \varepsilon_{j} R_{j j} .
$$

Recall that $r_{n}(z):=m_{n}(z)-s(z)$. The representation (3.7) implies

$r_{n}(z)=\frac{r_{n}(z)}{(z+s(z))\left(z+m_{n}(z)\right)}-\frac{\delta_{n}^{\prime}}{\left(z+m_{n}(z)\right)^{2}}+\frac{\widehat{\delta}_{n}}{z+m_{n}(z)}+\frac{\bar{\delta}_{n}}{\left(z+m_{n}(z)\right)^{2}}$.

The equality (6.2) yields

$$
\left|r_{n}(z)\right| \leq \frac{\left|\delta_{n}^{\prime}\right|+\left|\bar{\delta}_{n}\right|}{\left|z+m_{n}(z)\right|\left|z+s(z)+m_{n}(z)\right|}+\frac{\left|\widehat{\delta}_{n}\right|}{\left|z+s(z)+m_{n}(z)\right|} .
$$

By Lemmas 5.1, 5.3, 5.4 and 5.5, we get

$$
\operatorname{Pr}\left\{\left|r_{n}(z)\right| \leq \frac{C l_{n, \alpha}^{\frac{4}{\varkappa}} \psi_{0}}{n v\left|z+m_{n}(z)+s(z)\right|}+\frac{l_{n, \alpha}^{\frac{4}{\varkappa}}}{n v}\right\} \geq 1-\exp \left\{-c l_{n, \alpha}\right\}-\operatorname{Pr}\left\{\mathcal{U}^{c}\right\}
$$

We prove now following

Proposition 6.1. Assuming the assumption of Theorem 1.1, the following inequality holds

$$
\operatorname{Pr}\left\{\left|r_{n}(z)\right| \geq \frac{C \beta_{n}^{4}}{n v}+\frac{C \beta_{n}^{4}}{n^{2} v^{2}\left|z+m_{n}(z)+s(z)\right|}\right\} \leq \exp \left\{-c l_{n, \alpha}\right\} .
$$

Proof. First we put $\psi_{0}=\frac{3}{2}$. Since $\mathcal{A}_{v_{0}} \subset \mathcal{U}$ by Corollary 3.7 we get

$$
\operatorname{Pr}\left\{\mathcal{U}^{c}\right\} \leq \exp \left\{-c l_{n, \alpha}\right\} .
$$

By inequality (6.4), we have

$$
\operatorname{Pr}\left\{\left|r_{n}(z)\right| \leq \frac{C l_{n, \alpha}^{\frac{4}{\varkappa}}}{\left|z+m_{n}(z)+s(z)\right| n v}\right\} \geq 1-\exp \left\{-c l_{n, \alpha}\right\} .
$$

Note that $\left|z+m_{n}(z)+s(z)\right| \geq \operatorname{Im} m_{n}(z)$. Assume that

$$
\operatorname{Im} m_{n}(z) \geq \operatorname{Im} s(z)+\frac{C^{\frac{1}{2}} l_{n, \alpha}^{\frac{2}{\varkappa}}}{\sqrt{n v}}
$$


Then we get

$$
\left|r_{n}(z)\right| \leq \frac{\sqrt{C} l_{n, \alpha}^{\frac{2}{\varkappa}}}{\sqrt{n v}}
$$

This inequality implies that

$$
\operatorname{Pr}\left\{\operatorname{Im} m_{n}(z) \geq \operatorname{Im} s(z)+\frac{C l_{n, \alpha}^{\frac{2}{\varkappa}}}{\sqrt{n v}}\right\} \leq \exp \left\{-c l_{n, \alpha}\right\} .
$$

Put now $\psi_{0}=\operatorname{Im} s(z)+\frac{C l_{n, \alpha}^{\frac{2}{\hbar}}}{\sqrt{n v}}$. Note that for $|u| \leq 2, \operatorname{Im} s(z) \geq c \sqrt{v}$, and for $v \geq v_{0}, \psi_{0} \geq \frac{1}{n v}$. That means, that we may apply inequality (6.4) with $\psi_{0}=\operatorname{Im} s(z)+\frac{C l_{n, \alpha}^{\frac{1}{\varkappa}}}{\sqrt{n v}}$. Using that $\left|z+s(z)+m_{n}(z)\right| \geq \operatorname{Im} s(z)$, we get

$$
\operatorname{Im} m_{n}(z) \leq \operatorname{Im} s(z)+\frac{C l_{n, \alpha}^{\frac{2}{\varkappa}}}{n^{2} v^{2} \sqrt{\operatorname{Im} m_{n}(z)}}+\frac{C l_{n, \alpha}^{\frac{2}{\varkappa}}}{n v} .
$$

From this inequality it follows that

$$
\operatorname{Im} m_{n}(z) \leq \operatorname{Im} s(z)+\frac{C l_{n, \alpha}^{\frac{2}{\varkappa}}}{n v},
$$

with some other constant $C$. We put now $\psi_{0}=\operatorname{Im} s(z)+\frac{C l_{n, \alpha}^{\frac{2}{\varkappa}}}{n v}$. Thus have proved that

$$
\operatorname{Pr}\left\{\mathcal{U}^{c}\right\} \leq \exp \left\{-c l_{n, \alpha}\right\} .
$$

Applying inequality (6.4) again, Proposition 6.1 is proved.

Note that using a union bound we may prove that

$$
\begin{aligned}
\operatorname{Pr}\left\{\left|r_{n}(z)\right|\right. & \left.\leq \frac{C l_{n, \alpha}^{2 \varkappa} \psi_{0}}{\left|z+m_{n}(z)+s(z)\right|}+\frac{l_{n, \alpha}^{\frac{2}{\varkappa}}}{n v}, \text { for any } z=u+i v,|u| \leq 2, V \geq v \geq v_{0}\right\} \\
& \leq \exp \left\{-c l_{n, \alpha}\right\} .
\end{aligned}
$$

\section{Proof of Theorem 1.1}

To conclude the proof of Theorem [1.1] we shall now apply the result of Corollary 2.2 with $v_{0}=\frac{C_{0} \beta_{n}^{4}}{n}$ and $V=4$ to the empirical spectral distribution function $\mathcal{F}_{n}(x)$ of the random matrix $\mathbf{X}$. At first we bound the integral over the 
line $V=4$. Note that in this case we have $\left|z+m_{n}(z)\right| \geq 1$ and $\left|r_{n}(z)\right| \leq \frac{1}{2}$ a.s. Moreover, $\operatorname{Im} m_{n}^{(j)}(z) \leq \frac{1}{V} \leq \frac{1}{2}$. In this case the event $\mathcal{B}$ implies

$$
\left|R_{j j}^{(\mathbb{A})}\right|^{-1} \leq C|u|
$$

and

$$
\left|R_{j j}^{(\mathbb{A}}\right| \leq \frac{C}{|u|}
$$

for sufficiently large $u$ uniformly in $n$. We may prove that, for $u \in \mathbb{R}$

$$
\operatorname{Pr}\left\{\left|r_{n}(z)\right| \geq \frac{C \beta_{n}^{2}}{n\left(|u|^{2}+1\right)}\right\} \leq \exp \left\{-c l_{n, \alpha}\right\}
$$

This inequality implies that

$$
\operatorname{Pr}\left\{\int_{-\infty}^{\infty}\left|r_{n}(u+i V)\right| d u \leq \frac{C \beta_{n}^{2}}{n}\right\} \geq 1-\exp \left\{-c l_{n, \alpha}\right\}
$$

Consider now $u \in \mathbb{J}_{\varepsilon}$, where $\varepsilon=v_{0}^{\frac{2}{3}}$. By Proposition 6.1, we have

$$
\operatorname{Pr}\left\{\left|r_{n}(z)\right| \leq \frac{C l_{n, \alpha}^{2 \varkappa}}{n^{2} v^{2}\left|z+m_{n}(z)+s(z)\right|}+\frac{l_{n, \alpha}^{\frac{2}{\varkappa}}}{n v}\right\} \geq 1-\exp \left\{-c l_{n, \alpha}\right\} .
$$

Note that

$$
\left|z+m_{n}(z)+s(z)\right| \geq \operatorname{Im}(z+s(z)) \geq c \sqrt{\gamma+v},
$$

where $\gamma=2-|u|$. Integrating now in $v \in\left[v_{0} / \sqrt{\gamma}, V\right]$, we get

$$
\int_{v_{0} / \sqrt{\gamma}}^{V}\left|r_{n}(u+i v)\right| d v \leq \frac{C \beta_{n}^{2} \log n}{n}+\frac{C \beta_{n}^{2}}{n^{2} v_{0}} .
$$

Thus, Theorem 1.1 is proved.

\section{Proof of Theorem 1.3}

We may express the diagonal entries of the resolvent matrix $\mathbf{R}$ as follows

$$
R_{j j}=\sum_{k=1}^{n} \frac{1}{\lambda_{k}-z}\left|u_{j k}\right|^{2}
$$


Consider the distribution function, say $F_{n j}(x)$, of the probability distribution of the eigenvalues $\lambda_{k}$

$$
F_{n j}(x)=\sum_{k=1}^{n}\left|u_{j k}\right|^{2} \mathbb{I}\left\{\lambda_{k} \leq x\right\} .
$$

Then we have

$$
R_{j j}=R_{j j}(z)=\int_{-\infty}^{\infty} \frac{1}{x-z} d F_{n j}(x),
$$

which means that $R_{j j}$ is the Stieltjes transform of the distribution $F_{n j}(x)$. Note that, for any $\lambda>0$,

$$
\max _{1 \leq k \leq n}\left|u_{j k}\right|^{2} \leq \sup _{x}\left(F_{n j}(x+\lambda)-F_{n j}(x)\right)=: Q_{n j}(\lambda) .
$$

On the other hand, it is easy to check that

$$
Q_{n j}(\lambda) \leq 2 \sup _{u} \lambda \operatorname{Im} R_{j j}(u+i \lambda) .
$$

By Corollary 3.2 we obtain, for any $v \geq v_{0}$ with $v_{0}=\frac{C_{0} \beta_{n}^{4}}{n}$ with a sufficiently large constant $C_{0}$, and for any $u \in \mathbb{R}$,

$$
\operatorname{Pr}\left\{\left|R_{j j}\right| \geq 3\right\} \leq C \exp \left\{-c l_{n, \alpha}\right\},
$$

with constants $C$ and $c$ depending on $\varkappa, \alpha$. From here using a union bound it is straightforward to check that

$$
\operatorname{Pr}\left\{\sup _{u}\left|R_{j j}\left(u+i V_{0}\right)\right| \geq 3\right\} \leq C \exp \left\{-c l_{n, \alpha}\right\},
$$

with some other constants $C, c>0$. Putting $\lambda=v_{0}$ in the inequality (8.2), we obtain

$$
\operatorname{Pr}\left\{\max _{1 \leq k \leq n}\left|u_{j k}\right|^{2} \geq \frac{C \beta_{n}^{4}}{n}\right\} \leq C \exp \left\{-c l_{n, \alpha}\right\} .
$$

By an additional union bound we arrive at the inequality (1.7). To prove inequality (1.8), we consider the quantity $\rho_{j}:=R_{j j}-s(z)$. Using equalities (3.3) and (2.3), we get

$$
\rho_{j}=-\frac{s(z) r_{n}(z)}{z+m_{n}(z)}+\frac{\varepsilon_{j}}{z+m_{n}(z)} R_{j j} .
$$

By Proposition [6.1, and (5.12), we have, for $v \geq v^{\prime}:=C n^{-\frac{1}{2}}$

$$
\operatorname{Pr}\left\{\left|\rho_{j}\right| \leq \frac{c \beta_{n}^{2}}{\sqrt{n v}}\right\} \geq 1-C \exp \left\{-c l_{n, \alpha}\right\}
$$


From here it follows that

$$
\sup _{x \in \mathbb{J}_{\varepsilon}} \int_{v^{\prime}}^{V}\left|r_{j}(x+i v)\right| d v \leq \frac{C}{\sqrt{n}} .
$$

Similar to (7.4) we get

$$
\int_{-\infty}^{\infty}\left|\rho_{j}(x+i V)\right| d x \leq \frac{C \beta_{n}^{2}}{\sqrt{n}}
$$

Applying Corollary 2.2, we get

$$
\operatorname{Pr}\left\{\sup _{x}\left|F_{n j}(x)-G(x)\right| \leq \frac{\beta_{n}^{2}}{\sqrt{n}}\right\} \geq 1-C \exp \left\{-c l_{n, \alpha}\right\} .
$$

Using now that

$$
\operatorname{Pr}\left\{\sup _{x}\left|\mathcal{F}_{n}(x)-G(x)\right| \leq \frac{\beta_{n}^{4} \log n}{n}\right\} \geq 1-C \exp \left\{-c l_{n, \alpha}\right\},
$$

we get

$$
\operatorname{Pr}\left\{\sup _{x}\left|F_{n j}(x)-\mathcal{F}_{n}(x)\right| \leq \frac{\beta_{n}^{2}}{\sqrt{n}}\right\} \geq 1-C \exp \left\{-c l_{n, \alpha}\right\} .
$$

Thus, Theorem 1.3 is proved.

\section{Appendix}

\subsection{Proof of Remark 1.2}

Proof of Remark 1.2. Let $x \in\left[0, \frac{1}{2}\right]$. Denote by $\tau$ a random variable which is uniformly distributed on $[0,1]$ and use Taylor's formula to show

$$
G^{-1}(x)=-2+\mathbf{E}_{\tau} \frac{2 \pi x}{\sqrt{4-\left(G^{-1}(x \tau)\right)^{2}}} .
$$

By monotonicity of $G^{-1}(x)$, we get

$$
2+G^{-1}(x) \geq \frac{C x}{\sqrt{4-\left(G^{-1}(x)\right)^{2}}} .
$$

Using $\sqrt{4-y^{2}}=\sqrt{2+y} \sqrt{2-y}, y=G^{-1}(x)$, there is another absolute constant $C>0$ such that

$$
2+G^{-1}(x) \geq C x^{\frac{2}{3}}
$$


From the last inequality we get

$$
\sqrt{2+G^{-1}(\tau x)} \geq c(\tau x)^{1 / 3}
$$

and hence by (9.1) it follows that

$$
2+G^{-1}(x) \leq c^{\prime} x^{\frac{2}{3}} \mathbf{E}_{\tau} \frac{1}{\tau^{\frac{1}{3}}} \leq C_{2} x^{\frac{2}{3}},
$$

with some absolute constants $c^{\prime}, C_{2}>0$. Similarly for $x \in\left[\frac{1}{2}, 1\right]$ we get

$$
C_{1}(1-x)^{\frac{2}{3}} \leq 2-G^{-1}(x) \leq C_{2}(1-x)^{\frac{2}{3}} .
$$

Summarizing, we may write for another absolute constant $C_{1}$,

$$
C_{1} \min \left\{x^{\frac{2}{3}},(1-x)^{\frac{2}{3}}\right\} \leq 4-\left(G^{-1}(x)\right)^{2} \leq C_{1} \min \left\{x^{\frac{2}{3}},(1-x)^{\frac{2}{3}}\right\} .
$$

Furthermore,

$$
\Delta_{n}^{*}=\sup _{x}\left|\mathcal{F}_{n}(x)-G(x)\right|=\max _{1 \leq k \leq n}\left|\mathcal{F}_{n}\left(\lambda_{k}\right)-G\left(\lambda_{k}\right)\right|=\max _{1 \leq k \leq n}\left|\frac{k}{n}-G\left(\lambda_{k}\right)\right| .
$$

This implies that, for $\lambda_{k} \in[-2,2]$ and $|\theta| \leq 1$,

$$
\lambda_{k}=G^{-1}\left(\frac{k}{n}+\theta \Delta_{n}^{*}\right) .
$$

By Taylor's formula we have

$$
G^{-1}\left(\frac{k}{n}+\theta \Delta_{n}^{*}\right)=G^{-1}\left(\frac{k}{n}\right)+\mathbf{E}_{\tau} \frac{2 \pi \theta \Delta_{n}^{*}}{\sqrt{4-\left(G^{-1}\left(\frac{k}{n}+\tau \theta \Delta_{n}^{*}\right)\right)^{2}}} .
$$

Consider first the case $2 \Delta_{n}^{*} \leq \frac{k}{n} \leq \frac{1}{2}-\Delta_{n}^{*}$. Then by (9.7),

$$
\sqrt{4-\left(G^{-1}\left(\frac{k}{n}+\tau \theta \Delta_{n}^{*}\right)\right)^{2}} \geq C\left|\frac{k}{n}+\tau \theta \Delta_{n}^{*}\right|^{\frac{1}{3}} \geq C^{\prime}\left(\frac{k}{n}\right)^{\frac{1}{3}} .
$$

From here it follows that by Theorem 1.1, with probability $1-C \exp \left\{-c l_{n, \alpha}\right\}$,

$$
\left|\lambda_{k}-\gamma_{n k}\right| \leq C \beta_{n}^{4} n^{-\frac{2}{3}} k^{-\frac{1}{3}} .
$$

Similar we get, for $2 \Delta_{n}^{*} \leq \frac{n-k}{n} \leq \frac{1}{2}-\Delta_{n}^{*}$,

$$
\left|\lambda_{k}-\gamma_{n k}\right| \leq C \beta_{n}^{4} n^{-\frac{2}{3}}(n-k)^{-\frac{1}{3}} .
$$

Thus Remark 1.2 is proved. 


\subsection{Proof of Bounds for Kolmogorov Distance}

Proof of Proposition [2.1. The proof of Proposition 2.1 is a straightforward adaptation of the proof of Lemma 2.1 from [11]. We include it here for the sake of completeness. First we note that

$$
\begin{aligned}
\sup _{x}|F(x)-G(x)| & =\sup _{x \in[-2,2]}|F(x)-G(x)|=\max \left\{\sup _{x \in \mathbb{J}_{\varepsilon}}|F(x)-G(x)|,\right. \\
& \left.\sup _{x \in[-2,-2+\varepsilon]}|F(x)-G(x)|, \sup _{x \in[2-\varepsilon, 2]}|F(x)-G(x)|\right\} .
\end{aligned}
$$

Furthermore, for $x \in[-2,-2+\varepsilon]$ we have

$$
\begin{aligned}
-G(-2+\varepsilon) \leq F(x)-G(x) \leq F & (-2+\varepsilon)-G(-2+\varepsilon)+G(-2+\varepsilon) \\
& \leq \sup _{x \in \mathbb{J}_{\varepsilon}}|F(x)-G(x)|+G(-2+\varepsilon) .
\end{aligned}
$$

This inequality yields

$$
\sup _{x \in[-2,-2+\varepsilon]}|F(x)-G(x)| \leq \sup _{x \in \mathbb{J}_{\varepsilon}}|F(x)-G(x)|+G(-2+\varepsilon) .
$$

Similarly we get

$$
\sup _{x \in[2-\varepsilon, 2]}|F(x)-G(x)| \leq \sup _{x \in \mathbb{J}_{\varepsilon}}|F(x)-G(x)|+1-G(2-\varepsilon) .
$$

Note that $G(-2+\varepsilon)=1-G(2-\varepsilon)$ and $G(-2+\varepsilon) \leq C \varepsilon^{\frac{3}{2}}$ with some absolute constant $C>0$. Combining all these relations we get

$$
\sup _{x}|F(x)-G(x)| \leq \Delta_{\varepsilon}(F, G)+C \varepsilon^{\frac{3}{2}},
$$


where $\Delta_{\varepsilon}(F, G)=\sup _{x \in \mathbb{J}_{\varepsilon}}|F(x)-G(x)|$. We denote $v^{\prime}=\frac{v}{\sqrt{\gamma}}$. For any $x \in \mathbb{J}_{\varepsilon}^{\prime}$

$$
\begin{aligned}
\mid \frac{1}{\pi} & \operatorname{Im}\left(\int_{-\infty}^{x}\left(S_{F}\left(u+i v^{\prime}\right)-S_{G}\left(u+i v^{\prime}\right)\right) d u\right) \mid \\
& \geq \frac{1}{\pi} \operatorname{Im}\left(\int_{-\infty}^{x}\left(S_{F}\left(u+i v^{\prime}\right)-S_{G}\left(u+i v^{\prime}\right)\right) d u\right) \\
& =\frac{1}{\pi}\left[\int_{-\infty}^{x} \int_{-\infty}^{\infty} \frac{v^{\prime} d(F(y)-G(y))}{(y-u)^{2}+v^{\prime 2}} d u\right] \\
& =\frac{1}{\pi} \int_{-\infty}^{x}\left[\int_{-\infty}^{\infty} \frac{2 v^{\prime}(y-u)(F(y)-G(y)) d y}{\left((y-u)^{2}+v^{\prime 2}\right)^{2}}\right] \\
& =\frac{1}{\pi} \int_{-\infty}^{\infty}(F(y)-G(y))\left[\int_{-\infty}^{x} \frac{2 v^{\prime}(y-u)}{\left((y-u)^{2}+v^{\prime 2}\right)^{2}} d u\right] d y \\
& =\frac{1}{\pi} \int_{-\infty}^{\infty} \frac{F\left(x-v^{\prime} y\right)-G\left(x-v^{\prime} y\right)}{y^{2}+1} d y, \quad \text { by change of variables. }
\end{aligned}
$$

Furthermore, using (2.1) and the definition of $\Delta(F, G)$ we note that

$$
\frac{1}{\pi} \int_{|y|>a} \frac{\left|F\left(x-v^{\prime} y\right)-G\left(x-v^{\prime} y\right)\right|}{y^{2}+1} d y \leq(1-\beta) \Delta(F, G) .
$$

Since $F$ is non decreasing, we have

$$
\begin{gathered}
\frac{1}{\pi} \int_{|y| \leq a} \frac{F\left(x-v^{\prime} y\right)-G\left(x-v^{\prime} y\right)}{y^{2}+1} d y \geq \frac{1}{\pi} \int_{|y| \leq a} \frac{F\left(x-v^{\prime} a\right)-G\left(x-v^{\prime} y\right)}{y^{2}+1} d y \\
\geq\left(F\left(x-v^{\prime} a\right)-G\left(x-v^{\prime} a\right)\right) \beta \\
\quad-\frac{1}{\pi} \int_{|y| \leq a}\left|G\left(x-v^{\prime} y\right)-G\left(x-v^{\prime} a\right)\right| d y .
\end{gathered}
$$

These inequalities together imply (using a change of variables in the last step)

$$
\begin{aligned}
& \frac{1}{\pi} \int_{-\infty}^{\infty} \frac{F\left(x-v^{\prime} y\right)-G\left(x-v^{\prime} y\right)}{y^{2}+1} d y \geq \beta\left(F\left(x-v^{\prime} a\right)-G\left(x-v^{\prime} a\right)\right) \\
& \quad-\frac{1}{\pi} \int_{|y| \leq a}\left|G\left(x-v^{\prime} y\right)-G\left(x-v^{\prime} a\right)\right| d y-(1-\beta) \Delta(F, G) \\
& \quad \geq \beta\left(F\left(x-v^{\prime} a\right)-G\left(x-v^{\prime} a\right)\right) \\
& \quad-\frac{1}{v^{\prime} \pi} \int_{|y| \leq v^{\prime} a}\left|G(x-y)-G\left(x-v^{\prime} a\right)\right| d y-(1-\beta) \Delta(F, G) .
\end{aligned}
$$


Note that according to Remark 2.2, $x \pm v^{\prime} a \in \mathbb{J}_{\varepsilon}^{\prime}$ for any $x \in \mathbb{J}_{\varepsilon}$. Assume first that $x_{n} \in \mathbb{J}_{\varepsilon}$ is a sequence such that $F\left(x_{n}\right)-G\left(x_{n}\right) \rightarrow \Delta_{\varepsilon}(F, G)$. Then $x_{n}^{\prime}:=x_{n}+v^{\prime} a \in \mathbb{J}_{\varepsilon}^{\prime}$. Using (9.19) and (9.22), we get

$$
\begin{aligned}
\sup _{x \in \mathbb{J}_{\varepsilon}^{\prime}}\left|\operatorname{Im} \int_{-\infty}^{x}\left(S_{F}\left(u+i v^{\prime}\right)-S_{G}\left(u+i v^{\prime}\right)\right) d u\right| \\
\geq \operatorname{Im} \int_{-\infty}^{x_{n}^{\prime}}\left(S_{F}\left(u+i v^{\prime}\right)-S_{G}\left(u+i v^{\prime}\right)\right) d u \\
\geq \beta\left(F\left(x_{n}^{\prime}-v^{\prime} a\right)-G\left(x_{n}^{\prime}-v^{\prime} a\right)\right) \\
\quad \quad-\frac{1}{\pi v} \sup _{x \in \mathbb{J}_{\varepsilon}^{\prime}} \sqrt{\gamma} \int_{|y| \leq 2 v^{\prime} a}|G(x+y)-G(x)| d y-(1-\beta) \Delta(F, G) \\
=\beta\left(F\left(x_{n}\right)-G\left(x_{n}\right)\right) \\
-\frac{1}{\pi v} \sup _{x \in \mathbb{J}_{\varepsilon}^{\prime}} \sqrt{\gamma} \int_{|y|<2 v^{\prime} a}|G(x+y)-G(x)| d y-(1-\beta) \Delta(F, G) .
\end{aligned}
$$

Assume for definiteness that $y>0$. Recall that $\varepsilon \leq 2 \gamma$, for any $x \in \mathbb{J}_{\varepsilon}^{\prime}$. By Remark 2.2 with $\varepsilon / 2$ instead of $\varepsilon$, we have $0<y \leq 2 v^{\prime} a \leq \sqrt{2} \varepsilon$, for any $x \in \mathbb{J}_{\varepsilon}^{\prime}$. For the semi-circular law we have,

$$
\begin{aligned}
|G(x+y)-G(x)| & \leq y \sup _{u \in[x, x+y]} G^{\prime}(u) \leq y C \sqrt{\gamma+y} \\
& \leq C y \sqrt{\gamma+2 v^{\prime} a} \leq C y \sqrt{\gamma+\varepsilon} \leq C y \sqrt{\gamma} .
\end{aligned}
$$

This yields after integrating in $y$

$$
\frac{1}{\pi v} \sup _{x \in \mathbb{J}_{\varepsilon}^{\prime}} \sqrt{\gamma} \int_{0 \leq y \leq 2 v^{\prime} a}|G(x+y)-G(x)| d y \leq \frac{C}{v} \sup _{x \in \mathbb{J}_{\varepsilon}^{\prime}} \gamma v^{\prime 2} \leq C v .
$$

Similarly we get that

$$
\frac{1}{\pi v} \sup _{x \in \mathbb{J}_{\varepsilon}^{\prime}} \sqrt{\gamma} \int_{0 \geq y \geq-2 v^{\prime} a}|G(x+y)-G(x)| d y \leq \frac{C}{v} \sup _{x \in \mathbb{J}_{\varepsilon}^{\prime}} \gamma v^{\prime 2} \leq C v .
$$

By inequality (9.18)

$$
\Delta_{\varepsilon}(F, G) \geq \Delta(F, G)-C \varepsilon^{\frac{3}{2}} .
$$

The inequalities (9.23), (9.27) and (9.25), (9.26) together yield as $n$ tends to infinity

$$
\begin{gathered}
\sup _{x \in \mathbb{J}_{\varepsilon}^{\prime}}\left|\operatorname{Im} \int_{-\infty}^{x}\left(S_{F}\left(u+i v^{\prime}\right)-S_{G}\left(u+i v^{\prime}\right)\right) d u\right| \\
\geq(2 \beta-1) \Delta(F, G)-C v-C \varepsilon^{\frac{3}{2}},
\end{gathered}
$$


for some constant $C>0$. Similar arguments may be used to prove this inequality in case there is a sequence $x_{n} \in \mathbb{J}_{\varepsilon}$ such $F\left(x_{n}\right)-G\left(x_{n}\right) \rightarrow$ $-\Delta_{\varepsilon}(F, G)$. In view of (9.28) and $2 \beta-1=1 / 2$ this completes the proof.

\subsection{Proof of Lemma 3.1}

Proof. First we consider $m_{n}(z)-\widehat{m}_{n}(z)$. Denote by

$$
\widehat{\mathbf{R}}=(\widehat{W}-z \mathbf{I})^{-1} \text {. }
$$

We have

$$
m_{n}(z)-\widehat{m}_{n}(z)=\frac{1}{n} \operatorname{Tr} \mathbf{R}(\mathbf{W}-\widehat{\mathbf{W}}) \widehat{\mathbf{R}} .
$$

This representation and inequality $\max \{\|\mathbf{R}\|,\|\widehat{\mathbf{R}}\|\} \leq v^{-1}$ imply

$$
\left|m_{n}(z)-\widehat{m}_{n}(z)\right| \leq \frac{1}{\sqrt{n} v^{2}}\|\mathbf{W}-\widehat{\mathbf{W}}\|_{2}=v^{-2}\left(\frac{1}{n^{2}} \sum_{j, l=1}^{n}\left|X_{j l}-\widehat{X}_{j l}\right|^{2}\right)^{\frac{1}{2}}
$$

From here it follows that

$$
\operatorname{Pr}\left\{\left|m_{n}(z)-\widehat{m}_{n}(z)\right|>\frac{C}{n^{2} v^{2}}\right\} \leq \sum_{j, l=1}^{n} \operatorname{Pr}\left\{\left|X_{j l}-\widehat{X}_{j l}\right|>\frac{C}{n^{2}}\right\} .
$$

Note that

$$
X_{j l}-\widehat{X}_{j l}=X_{j l} \mathbb{I}\left\{\left|X_{j l}\right| \geq C l_{n, \alpha}^{\frac{1}{\varkappa}}\right\}-\mathbf{E} X_{j l} \mathbb{I}\left\{\left|X_{j l}\right| \geq C l_{n, \alpha}^{\frac{1}{\varkappa}}\right\} .
$$

Condition (1.1) implies that

$$
\left|\mathbf{E} X_{j l} \mathbb{I}\left\{\left|X_{j l}\right| \geq C l_{n, \alpha}^{\frac{1}{\varkappa}}\right\}\right| \leq \exp \left\{-c l_{n, \alpha}\right\} \leq \frac{C}{2 n^{2}} .
$$

From here it follows that

$$
\operatorname{Pr}\left\{\left|X_{j l}-\widehat{X}_{j l}\right|>\frac{C}{n^{2}}\right\} \leq \operatorname{Pr}\left\{\left|X_{j l}\right| \geq C l_{n, \alpha}^{\frac{1}{\varkappa}}\right\} \leq A \exp \left\{-c l_{n, \alpha}\right\} .
$$

Inequalities (9.32) and (9.35) together imply that there exists a constant $c^{\prime}$ such that

$$
\operatorname{Pr}\left\{\left|m_{n}(z)-\widehat{m}_{n}(z)\right|>\frac{C}{n^{2} v^{2}}\right\} \leq \exp \left\{-c^{\prime} l_{n, \alpha}\right\} .
$$


We prove now that

$$
\operatorname{Pr}\left\{\left|\widetilde{m}_{n}(z)-\widehat{m}_{n}(z)\right| \geq \frac{C}{n^{2} v^{2}}\right\} \leq \exp \left\{-c l_{n, \alpha}\right\} .
$$

Repeating the arguments of (3.1) - (9.35), we need to prove

$$
\operatorname{Pr}\left\{\left|\widehat{X}_{j k}-\widetilde{X}_{j k}\right|>\frac{C}{n^{3}}\right\} \leq \operatorname{Pr}\left\{\left(1-\sigma_{j k}\right) \sigma_{j k}^{-1}\left|\widehat{X}_{j k}\right|>\frac{C}{n^{2}}\right\} .
$$

Note that

$$
\sigma_{j k}^{2}=1-\mathbf{E} X_{j k}^{2} \mathbb{I}\left\{\left|X_{j k}\right| \geq c l_{n, \alpha}^{\frac{1}{\varkappa}}\right\}-\left(\mathbf{E} X_{j k} \mathbb{I}\left\{\left|X_{j k}\right| \geq c l_{n, \alpha}^{\frac{1}{\varkappa}}\right\}\right)^{2} \geq 1-\exp \left\{-c^{\prime} l_{n, \alpha}\right\} .
$$

The last bound is uniform in $j, k=1, \ldots, n$. This implies that

$$
\left(1-\sigma_{j k}\right) \sigma_{j k}^{-1} \leq \exp \left\{-c^{\prime \prime} l_{n, \alpha}\right\} .
$$

Inequalities (9.38) and (9.40) together imply (9.37). Thus Lemma 3.1 is proved.

\subsection{Proof of the Key Lemma}

Proof. Since $|s(z)| \leq 1$ for any $z \in \mathbb{C}_{+}$, we obtain that, for $\omega \in A_{k}$, for any $z=u+i v$ with $v \geq v_{k}$

$$
\left|m_{n}(z)\right| \leq|s(z)|+\left|m_{n}(z)-s(z)\right| \leq \frac{3}{2}
$$

and

$$
\left|z+m_{n}(z)\right| \geq|z+s(z)|-\left|m_{n}(z)-s(z)\right| \geq \frac{1}{2} .
$$

Furthermore,

$$
\left|m_{n}^{(j)}(z)\right| \leq\left|m_{n}(z)\right|+\frac{1}{n v} \leq 2,
$$

for any $z=u+i v$ with $v \geq v_{0}$. Note that for semi-circular law $|s(z)+z| \geq 1$ for any $z \in \mathbb{C}^{+}$. This implies that, for any $\omega \in \mathcal{A}_{k}$ and any $z=u+i v$ with $v \geq v_{k}$

$$
\left|z+m_{n}(z)\right| \geq|z+s(z)|-\left|m_{n}(z)-s(z)\right| \geq \frac{1}{2} .
$$

From here it follows that, for any $\omega \in \mathcal{A}_{k}$ and for any $z=u+i v$ with $v \geq v_{0}$, by (3.3), for any $j=1, \ldots, n$

$$
\left|R_{j j}\right| \leq 2\left(1+\gamma_{0}\left|R_{j j}\right|\right)
$$


which implies

$$
\left|R_{j j}\right| \leq 2\left(1-2 \gamma_{0}\right)^{-1} .
$$

From $1+z s(z)+s^{2}(z)=0($ see (2.3) $)$ ) we get $(z+s(z)) s(z)=-1$ and hence by representation (3.7)

$$
\begin{aligned}
\theta_{n}(z) & :=\frac{1}{n} \sum_{j=1}^{n} \varepsilon_{j} R_{j j}=-m_{n}(z)\left(z+m_{n}(z)\right)-1 \\
& =-\left(z+2 s(z)+m_{n}(z)-s(z)\right)\left(m_{n}(z)-s(z)\right) .
\end{aligned}
$$

From (3.5) it follows now

$$
\left|m_{n}(z)-s(z)\right| \leq \frac{2 \gamma_{0}}{\left(1-2 \gamma_{0}\right)\left|m_{n}(z)+s(z)+z\right|} .
$$

Assume that $\left|m_{n}(z)+s(z)+z\right| \geq \sqrt{\gamma_{0}}$. Then

$$
\left|m_{n}(z)-s(z)\right| \leq \frac{2 \sqrt{\gamma_{0}}}{1-2 \gamma_{0}} .
$$

Let now $\left|z+m_{n}(z)+s(z)\right| \leq \sqrt{\gamma_{0}}$. Assume that

$$
\left|m_{n}(z)-s(z)\right| \geq 2 \sqrt{\gamma_{0}} .
$$

Then

$$
|z+2 s(z)| \geq\left|s(z)-m_{n}(z)\right|-\left|z+m_{n}(z)+s(z)\right| \geq \sqrt{\gamma_{0}} .
$$

Since $\operatorname{Re}\left(z^{2}-4\right)<0$, for $|u| \leq 2$, we have

$$
\begin{aligned}
\left|z+m_{n}(z)+s(z)\right| & \geq \operatorname{Im}\left(z+m_{n}(z)+s(z)\right) \geq \frac{1}{2} \operatorname{Im}(z+2 s(z)) \\
& \geq \frac{1}{2 \sqrt{2}}\left|z^{2}-4\right|^{\frac{1}{2}}=\frac{1}{2 \sqrt{2}}|z+2 s(z)| \geq \frac{1}{2 \sqrt{2}} \sqrt{\gamma_{0}} .
\end{aligned}
$$

Inequalities (9.45) and (9.49) under assumption (9.47) together imply

$$
\left|m_{n}(z)-s(z)\right| \leq \frac{4 \sqrt{2 \gamma_{0}}}{1-2 \gamma_{0}} .
$$

Combining inequalities (9.46), (9.47) and (9.50) we get that (9.50) holds for any $\omega \in \mathcal{A}_{k}$. Simple calculations show that if $\gamma_{0} \leq \frac{3}{100}$ then

$$
\left|m_{n}(z)-s(z)\right| \leq \frac{1}{4}
$$


Furthermore, for any $v_{k} \geq v \geq v_{k-1}$,

$$
\left|s_{n}\left(u+i v_{k-1}\right)-s_{n}(u+i v)\right| \leq \frac{\left|v-v_{k-1}\right|}{v v_{k-1}} \leq \frac{1}{n^{2} v_{0}^{2}} \leq \frac{1}{4} .
$$

The last two inequalities together imply, for any $z=u+i v$ with $v \geq v_{k-1}$, $\left|m_{n}(z)-s(z)\right| \leq \frac{1}{2}$ or $\omega \in \mathcal{A}_{k-1}$. Thus the Lemma is proved.

Remark 9.1. The inequality (9.43) yields, for $\gamma_{0} \leq \frac{3}{100}$,

$$
\left|R_{j j}\right| \leq \frac{3}{2}
$$

\subsection{The Proof of Lemma 3.4}

Note that

$$
\operatorname{Pr}\left\{\mathcal{D}_{k}^{c} \cap \mathcal{A}_{k} \cap \mathcal{U}\right\} \leq \sum_{j=1}^{n} \sum_{\nu=1}^{4} \operatorname{Pr}\left\{\left\{\left|\varepsilon_{j \nu}\right| \geq \frac{1}{4} \gamma_{0}\right\} \cap \mathcal{A}_{k} \cap \mathcal{U}\right\} .
$$

First we note that according Remark 3.1 we may choose the constant $A_{0}$ such that

$$
\operatorname{Pr}\left\{\left|\varepsilon_{j 1}\right| \geq \frac{1}{4} \gamma_{0}\right\}=0 .
$$

Next, we have (see Lemma 4.1 in [11])

$$
\left|\varepsilon_{j 4}\right| \leq \frac{1}{n v} \text { a. s. }
$$

We may choose the constant $C_{0}$ in the definition of $v_{0}$ such that

$$
\operatorname{Pr}\left\{\left|\varepsilon_{j 4}\right| \geq \frac{1}{4} \gamma_{0}\right\}=0 \text {. }
$$

We shall bound $\varepsilon_{j \nu}$, for $\nu=2,3$ and $j=1, \ldots, n$. First we note that

$$
\begin{aligned}
\operatorname{Pr}\left\{\left\{\left|\varepsilon_{j \nu}\right|\right.\right. & \left.\left.>\frac{1}{4} \gamma_{0}\right\} \cap \mathcal{A}_{k} \cap \mathcal{U}\right\} \\
& \leq \operatorname{Pr}\left\{\left|\varepsilon_{j \nu}\right| \geq \frac{1}{4 \sqrt{\psi_{0}}} \gamma_{0}\left(\operatorname{Im} m_{n}^{(j)}(z)\right)^{\frac{1}{2}} ; \operatorname{Im} m_{n}^{(j)}(z) \leq \psi_{0}\right\} \\
& \leq \operatorname{Pr}\left\{\left|\varepsilon_{j \nu}\right| \geq \frac{1}{4 \sqrt{\psi_{0}}} \gamma_{0}\left(\operatorname{Im} m_{n}^{(j)}(z)\right)^{\frac{1}{2}}\right\} .
\end{aligned}
$$

We now estimate the last probability in the r.h.s of (9.57). 
We we shall use McDiarmid's inequality for sums of independent random variables and for martingales. Let $\xi_{1}, \ldots, \xi_{n}$ be martingale-differences such that $\mathbf{E} \xi_{j}=0$ and $\left|\xi_{j}\right| \leq \sigma_{j}$. Then, for some numerical constant $c>0$,

$$
\operatorname{Pr}\left\{\left|\sum_{j=1}^{n} \xi_{j}\right|>x\right\} \leq c(1-\Phi(x / \sigma)) \leq \frac{c \sigma}{x} \exp \left\{-\frac{x^{2}}{2 \sigma^{2}}\right\}
$$

where $\Phi(x)=\frac{1}{\sqrt{2 \pi}} \int_{-\infty}^{x} \exp \left\{-\frac{y^{2}}{2}\right\} d y$ and $\sigma^{2}=\sigma_{1}^{2}+\cdots+\sigma_{n}^{2}$. The last inequality holds for $x \geq \sigma$. (See, for instance [3], p.1, first inequality.) Consider $\nu=2$. We put $\eta_{l}=\frac{1}{n}\left(X_{j l}^{2}-1\right) R_{l l}^{(j)}$.

Note that $\mathbf{E} \eta_{l}=0$ and $\left|\eta_{l}\right| \leq 2 l_{n, \alpha}^{\frac{2}{\varkappa}} n^{-1}\left|R_{l l}^{(j)}\right|$. Recall that

$$
\varepsilon_{j 2}=\frac{1}{n} \sum_{l \in \mathbb{T}_{j}}\left(X_{j l}^{2}-1\right) R_{l l}^{(j)}=\frac{1}{n} \sum_{l \in \mathbb{T}_{j}} \eta_{l} .
$$

Recall that $\mathfrak{R}^{(j)}$ denotes the $\sigma$-algebra generated by the random variables $X_{k l}$ with $k, l \in \mathbb{T}_{j}$. Let $\mathbf{E}_{j}$ and $\operatorname{Pr}_{j}$ denote the conditional expectation and the conditional probability with respect to $\mathfrak{R}^{(j)}$. Note that the random variables $X_{j l}$ and the $\sigma$-algebra $\mathfrak{R}^{(j)}$ are independent. Applying inequality (9.58) with $x:=\frac{1}{4 \sqrt{\psi_{0}}} \gamma_{0}\left(\operatorname{Im} m_{n}^{(j)}(z)\right)^{\frac{1}{2}}$ and with $\sigma^{2}=4 l_{n, \alpha}^{\frac{4}{\varkappa}} n^{-1}\left(\frac{1}{n} \sum_{l \in \mathbb{T}_{j}}\left|R_{l l}^{(j)}\right|^{2}\right)$, we get

$$
\begin{aligned}
\operatorname{Pr}\left\{\left|\sum_{l \in \mathbb{T}_{j}} \eta_{j}\right|>x\right\} & =\operatorname{EPr}_{j}\left\{\left|\sum_{l \in \mathbb{T}_{j}} \eta_{j}\right| \geq x\right\} \\
& \leq \mathbf{E} \exp \left\{-\frac{x^{2}}{\sigma^{2}}\right\} \leq C \exp \left\{-c n v /\left(16 \psi_{0} l_{n, \alpha}^{\frac{4}{\varkappa}}\right)\right\} .
\end{aligned}
$$

Since

$$
\frac{1}{n} \sum_{l \in \mathbb{T}_{j}}\left|R_{l l}^{(j)}\right|^{2} \leq v^{-1} \operatorname{Im} m_{n}^{(j)}(z),
$$

we have used here

$$
\sigma^{2} \leq 4 l_{n, \alpha}^{\frac{4}{\varkappa}} n^{-1} v^{-1} \operatorname{Im} m_{n}^{(j)}(z) .
$$

Now we consider $\varepsilon_{j 3}$. We represent $\varepsilon_{j 3}=\frac{1}{n} \sum_{l \in \mathbb{T}_{j}} \alpha_{l}$ as a martingale with martingale difference

$$
\alpha_{l}=\sum_{1 \leq q \neq r \leq l} X_{j q} X_{j r} R_{q r}^{(j)}-\sum_{1 \leq q \neq r \leq l-1} X_{j q} X_{j r} R_{q r}^{(j)}=2 X_{j l} \sum_{q=1}^{l-1} X_{j q} R_{q l}^{(j)} .
$$


Because the $X_{j l}$ are bounded by $l_{n, \alpha}^{\frac{1}{\varkappa}}$, we may apply McDiarmid's inequality. This implies that

$$
\operatorname{Pr}\left\{\left|\alpha_{l}\right| \geq\left(n v_{k} / \psi_{0}\right)^{\frac{1}{4}} l_{n, \alpha}^{\frac{1}{\varkappa}}\left(\sum_{q \in \mathbb{T}_{j}}\left|R_{q l}^{(j)}\right|^{2}\right)^{\frac{1}{2}}\right\} \leq \exp \left\{-c \sqrt{n v_{k}} /\left(\sqrt{\psi_{0}} l_{n, \alpha}^{\frac{2}{\varkappa}}\right)\right\} .
$$

Furthermore, we note that

$$
\begin{array}{r}
\frac{1}{n} \sum_{l \in \mathbb{T}_{j}} \mathbf{E}\left\{\alpha_{l} \mathbb{I}\left\{\left|\alpha_{l}\right| \geq\left(n v_{k} / \psi_{0}\right)^{\frac{1}{4}} l_{n, \alpha}^{\frac{1}{\varkappa}}\left(\sum_{k \in \mathbb{T}_{j}}\left|R_{l k}^{(j)}\right|^{2}\right)^{\frac{1}{2}}\right\} \mid \mathfrak{M}_{l}^{(j)}\right\} \\
\leq \exp \left\{-c^{\prime} \sqrt{n v_{k}} /\left(\sqrt{\psi_{0}} l_{n, \alpha}^{\frac{2}{\varkappa}}\right)\right\}
\end{array}
$$

where $\mathfrak{M}_{l-1}^{(j)}$ denotes the $\sigma$-algebra generated by $X_{s k}$ with $s, k \in \mathbb{T}_{j}$ such that $1 \leq s, k \leq l-1$. We consider now the truncated martingale with

$$
\begin{aligned}
\widehat{\alpha}_{l}=\alpha_{l} \mathbb{I}\left\{\left|\alpha_{l}\right|\right. & \left.\leq\left(n v_{k} / \psi_{0}\right)^{\frac{1}{4}} l_{n, \alpha}^{\frac{1}{\varkappa}}\left(\sum_{t \in \mathbb{T}_{j}}\left|R_{l t}^{(j)}\right|^{2}\right)^{\frac{1}{2}}\right\} \\
& -\mathbf{E}\left\{\alpha \mathbb{I}\left\{|\alpha| \leq\left(n v_{k} / \psi_{0}\right)^{\frac{1}{4}} l_{n, \alpha}^{\frac{1}{\varkappa}}\left(\sum_{t \in \mathbb{T}_{j}}\left|R_{l t}^{(j)}\right|^{2}\right)^{\frac{1}{2}}\right\} \mid \mathfrak{M}_{l-1}^{(j)}\right\} .
\end{aligned}
$$

Applying McDiarmid's inequality for martingales (see [3]), we get

$$
\operatorname{Pr}\left\{\left|\sum_{l \in \mathbb{T}_{j}} \widehat{\alpha}_{l}\right| \geq x\left(\sum_{s, t \in \mathbb{T}_{j}}\left|R_{s t}^{(j)}\right|^{2}\right)^{\frac{1}{2}}\right\} \leq \exp \left\{-x^{2} \sqrt{\psi_{0}} /\left(\sqrt{n v_{k}} l_{n, \alpha}^{\frac{2}{\varkappa}}\right)\right\} .
$$

Putting $x=\frac{1}{4 \sqrt{\psi_{0}}} \gamma_{0} \sqrt{n v_{k}}$, we get

$$
\operatorname{Pr}\left\{\left|\sum_{l \in \mathbb{T}_{j}} \widehat{\alpha}_{l}\right| \geq \gamma_{n} \sqrt{n v_{k}}\left(\sum_{s, t \in \mathbb{T}_{j}}\left|R_{s t}^{(j)}\right|^{2}\right)^{\frac{1}{2}}\right\} \leq \exp \left\{-c \sqrt{n v_{k}} /\left(\sqrt{\psi_{0}} l_{n, \alpha}^{\frac{2}{\varkappa}}\right)\right\}
$$

We may rewrite the last inequality now as

$\operatorname{Pr}\left\{\left|\frac{1}{n} \sum_{l \in \mathbb{T}_{j}} \widehat{\alpha}_{l}\right| \geq \frac{1}{4 \sqrt{\psi_{0}}} \gamma_{0}\left(v_{k} \frac{1}{n} \sum_{s, t \in \mathbb{T}_{j}}\left|R_{s t}^{(j)}\right|^{2}\right)^{\frac{1}{2}}\right\} \leq \exp \left\{-c \sqrt{n v_{k}} /\left(\sqrt{\psi_{0}} l_{n, \alpha}^{\frac{2}{\varkappa}}\right)\right\}$.

Thus the Lemma is proved. 


\subsection{The proof of lemma 3.9}

We consider the case $\mathbb{A}=\emptyset$ only. We start with the following inequality

$$
\begin{aligned}
\mathbf{E}\left\{\left(\sum_{q \in \mathbb{T}_{j, l}}\left|R_{q l}\right|^{2}\right)^{m} \mathbb{I}\{\mathcal{U}\} \mid \mathfrak{M}^{(j)}\right\} & \leq 4^{m}\left(\sum_{q \in \mathbb{T}_{j}}\left|R_{q l}^{(j)}\right|^{2}\right)^{m} \mathbf{E}\left\{\mathbb{I}\{\mathcal{U}\} \mid \mathfrak{M}^{(j)}\right\} \\
& +4^{m} \mathbf{E}\left\{\left(\sum_{q \in \mathbb{T}_{j, l}}\left|R_{q l}-R_{q l}^{(j)}\right|^{2}\right)^{m} \mathbb{I}\{\mathcal{U}\} \mid \mathfrak{M}^{(j)}\right\}
\end{aligned}
$$

Using the equality (see, for instance [14], Lemma 3.2)

$$
R_{q l}-R_{q l}^{(j)}=R_{q j} R_{j l}\left(R_{j j}\right)^{-1}
$$

we get

$$
\begin{aligned}
\mathbf{E}\left\{\left(\sum_{q \in \mathbb{T}_{j, l}}\left|R_{q l}\right|^{2}\right)^{m}\right. & \left.\mathbb{I}\{\mathcal{U}\} \mid \mathfrak{M}^{(j)}\right\} \leq 4^{m}\left(\sum_{q \in \mathbb{T}_{j, l}}\left|R_{q l}^{(j)}\right|^{2}\right)^{m} \mathbf{E}\left\{\mathbb{I}\{\mathcal{U}\} \mid \mathfrak{M}^{(j)}\right\} \\
& +4^{m} \mathbf{E}\left\{\left(\sum_{q \in \mathbb{T}_{j, l}}\left|R_{q j}\right|^{2}\right)^{m}\left|R_{j l}\right|^{m}\left|R_{j j}\right|^{-m} \mathbb{I}\{\mathcal{U}\} \mid \mathfrak{M}^{(j)}\right\}
\end{aligned}
$$

Note that by (2.5)

$$
\sum_{q \in \mathbb{T}_{j, l}}\left|R_{q j}\right|^{2} \leq n v^{-2} \text { a.s. }
$$

and by (3.3)

$$
\left|\varepsilon_{j}\right| \leq \frac{C l_{n, \alpha}^{\frac{1}{\varkappa}}}{n}+\frac{1}{n v}+v^{-1} l_{n, \alpha}^{\frac{2}{\varkappa}}+l_{n, \alpha}^{\frac{2}{\varkappa}} \sqrt{n} v^{-1} \text { a.s. }
$$

These inequalities together imply that, for $v \geq v_{0}$

$$
\left|R_{j j}\right|^{-1} \leq\left|z+m_{n}(z)\right|\left(1+\left|\varepsilon_{j}\right|\left|R_{j j}\right|\right) \leq\left(|z|+v^{-1}\right)\left(1+n l_{n, \alpha}^{\frac{2}{\varkappa}} v^{-1}\right) \leq C n^{2} \text { a.s. }
$$

Applying these inequalities, we get

$$
\begin{aligned}
& \mathbf{E}\left\{\left(\sum_{q \in \mathbb{T}_{j, l}}\left|R_{q j}\right|^{2}\right)^{m}\left|R_{j l}\right|^{m}\left|R_{j j}\right|^{-m} \mathbb{I}\{\mathcal{U}\} \mid \mathfrak{M}^{(j)}\right\} \\
& \leq C^{m} \mathbf{E}\left\{\left(\sum_{q \in \mathbb{T}_{j, l}}\left|R_{q j}\right|^{2}\right)^{m}\left|R_{j l}\right|^{m} \mathbb{I}\{\mathcal{U}\} \mid \mathfrak{M}^{(j)}\right\}+C^{m} n^{3 m} \mathbf{E}\left\{\mathbb{I}\left\{\left(\mathcal{B}^{(l)}\right)^{c}\right\} \mathbb{I}\{\mathcal{U}\} \mid \mathfrak{M}^{(j)}\right\},
\end{aligned}
$$


where $\mathcal{B}^{(\mathbb{A})}$ was defined in (3.25). Applying Hölder's inequality, we get

$$
\begin{aligned}
\mathbf{E}\left\{\left(\sum_{q \in \mathbb{T}_{j, l}}\left|R_{q j}\right|^{2}\right)^{m}\left|R_{j l}\right|^{m} \mathbb{I}\{\mathcal{U}\} \mid \mathfrak{M}^{(j)}\right\} \leq & \mathbf{E}^{\frac{1}{2}}\left\{\left(\sum_{q \in \mathbb{T}_{j, l}}\left|R_{q j}\right|^{2}\right)^{2 m} \mathbb{I}\{\mathcal{U}\} \mid \mathfrak{M}^{(j)}\right\} \\
& \times \mathbf{E}^{\frac{1}{2}}\left\{\left|R_{j l}\right|^{2 m} \mathbb{I}\{\mathcal{U}\} \mid \mathfrak{M}^{(j)}\right\} .
\end{aligned}
$$

By Lemma 3.8, we have

$$
\mathbf{E}^{\frac{1}{2}}\left\{\left(\sum_{q \in \mathbb{T}_{j, l}}\left|R_{q j}\right|^{2}\right)^{2 m} \mathbb{I}\{\mathcal{U}\} \mid \mathfrak{M}^{(j)}\right\} \leq C m^{m}\left(l_{n, \alpha}^{\frac{2}{\varkappa}}\right)^{m} \psi_{0}^{m}+\Phi_{n} .
$$

The second factor is bounded, using equality (3.27). We get

$$
\begin{aligned}
& \mathbf{E}^{\frac{1}{2}}\left\{\left|R_{j l}\right|^{2 m} \mathbb{I}\{\mathcal{U}\} \mid \mathfrak{M}^{(j)}\right\} \leq \mathbf{E}^{\frac{1}{2}}\left\{\left|R_{j j}\right|^{2 m}\left|\frac{1}{\sqrt{n}} \sum_{q \in \mathbb{T}_{j, t}} X_{j q} R_{q l}^{(j)}\right|^{2 m} \mathbb{I}\{\mathcal{U}\} \mid \mathfrak{M}^{(j)}\right\} \\
& \quad \leq C^{2 m} \mathbf{E}^{\frac{1}{2}}\left\{\left|\frac{1}{\sqrt{n}} \sum_{q \in \mathbb{T}_{j, l}} X_{j q} R_{q l}^{(j)}\right|^{2 m} \mathbb{I}\{\mathcal{U}\} \mid \mathfrak{M}^{(j)}\right\}+\mathbf{E}^{\frac{1}{2}}\left\{\mathbb{I}\left\{(\mathcal{B})^{c} \mathbb{I}\{\mathcal{U}\}\right\} \mid \mathfrak{M}^{(j)}\right\} .
\end{aligned}
$$

Applying now Rosenthal's inequality, we obtain

$$
\begin{gathered}
\mathbf{E}^{\frac{1}{2}}\left\{\left|R_{j l}\right|^{2 m} \mathbb{I}\{\mathcal{U}\} \mid \mathfrak{M}^{(j)}\right\} \leq C^{m} m^{m}\left(\frac{1}{\sqrt{n}}\right)^{m}\left(\sum_{q \in \mathbb{T}_{j, l}}\left|R_{q l}^{(j)}\right|^{2}\right)^{\frac{m}{2}} \mathbf{E}\left\{\mathbb{I}\{\mathcal{U}\} \mid \mathfrak{M}^{(j)}\right\} \\
+\mathbf{E}^{\frac{1}{2}}\left\{\mathbb{I}\left\{\left(\mathcal{B}^{(l)}\right)^{c}\right\} \mathbb{I}\{\mathcal{U}\} \mid \mathfrak{M}^{(j)}\right\}
\end{gathered}
$$

Inequalities (9.75), (9.76), (9.77) together imply

$$
\begin{aligned}
\mathbf{E}\left\{\left(\sum_{q \in \mathbb{T}_{j, l}}\left|R_{q j}\right|^{2}\right)^{m}\left|R_{j l}\right|^{m} \mathbb{I}\{\mathcal{U}\} \mid \mathfrak{M}^{(j)}\right\} & \\
\leq & \left(\frac{C m^{2} l_{n, \alpha}^{\frac{2}{\varkappa}}}{\sqrt{n}}\right)^{m} \psi_{0}^{m}\left(\sum_{q \in \mathbb{T}_{j, l}}\left|R_{q l}^{(j)}\right|^{2}\right)^{\frac{m}{2}} \mathbf{E}\left\{\mathbb{I}\{\mathcal{U}\} \mid \mathfrak{M}^{(j)}\right\} \\
& +\left(C m l_{n, \alpha}^{\frac{2}{\varkappa}} v^{-1}\right)^{m} \mathbf{E}^{\frac{1}{2}}\left\{\mathbb{I}\left\{(\mathcal{B})^{c}\right\} \mathbb{I}\{\mathcal{U}\} \mid \mathfrak{M}^{(j)}\right\} .
\end{aligned}
$$

Inequalities (9.70) and (9.78) yield

$$
\begin{aligned}
\mathbf{E}\left\{\left(\sum_{q \in \mathbb{T}_{j, l}}\left|R_{q l}\right|^{2}\right)^{m} \mathbb{I}\{\mathcal{U}\} \mid \mathfrak{M}^{(j)}\right\} & \leq C^{m}\left(\sum_{q \in \mathbb{T}_{j l}}\left|R_{q l}^{(j)}\right|^{2}\right)^{m} \mathbf{E}\left\{\mathbb{I}\{\mathcal{U}\} \mid \mathfrak{M}^{(j)}\right\} \\
& +\left(\frac{C m^{2} l_{n, \alpha}^{\frac{2}{\varkappa}}}{\sqrt{n}}\right)^{m}\left(\sum_{q \in \mathbb{T}_{j l}}\left|R_{q l}^{(j)}\right|^{2}\right)^{\frac{m}{2}} \mathbf{E}\left\{\mathbb{I}\{\mathcal{U}\} \mid \mathfrak{M}^{(j)}\right\} \\
& +C^{m} n^{3 m} \mathbf{E}\left\{\mathbb{I}\left\{\left(\mathcal{B}^{(l)}\right)^{c}\right\} \mathbb{I}\{\mathcal{U}\} \mid \mathfrak{M}^{(j)}\right\} \\
& +C^{m} n^{2 m} \mathbf{E}^{\frac{1}{2}}\left\{\mathbb{I}\left\{(\mathcal{B})^{c}\right\} \mathbb{I}\{\mathcal{U}\} \mid \mathfrak{M}^{(j)}\right\}
\end{aligned}
$$


The last inequality completes the proof. Thus Lemma 3.9 is proved.

\subsection{The Proof of Lemma 3.10}

Note that

$$
\operatorname{Pr}\{\zeta \neq \widehat{\zeta}\} \leq \sum_{j \in \mathbb{T}} \operatorname{Pr}\left\{\mathbb{F}_{j}^{c}\right\}+n \operatorname{Pr}\left\{\mathcal{E}^{c}\right\}+\operatorname{Pr}\left\{\mathcal{U}^{c}\right\}
$$

By Corollary 3.5,

$$
\operatorname{Pr}\left\{\mathcal{E}^{c}\right\} \leq \exp \left\{-c \sqrt{n v_{0}} / l_{n, \alpha}\right\} \leq \exp \left\{-c l_{n, \alpha}\right\} .
$$

From here it follows that

$$
n \operatorname{Pr}\left\{\mathcal{E}^{c}\right\} \leq \exp \left\{-c^{\prime} l_{n, \alpha}\right\}
$$

We bound now $\operatorname{Pr}\left\{\left(\mathcal{F}_{j}\right)^{c}\right\}$ for $j=1, \ldots, n$. We use the same arguments as in (9.63) - 9.67). Recall $\varepsilon_{j 3}=\zeta_{j}=\frac{1}{n} \sum_{l \in \mathbb{T}_{j}} \alpha_{l}$ with

$$
\alpha_{l}=\sum_{1 \leq q \neq r \leq l} X_{j q} X_{j r} R_{q r}^{(j)}-\sum_{1 \leq q \neq r \leq l-1} X_{j q} X_{j r} R_{q r}^{(j)}=2 X_{j l} \sum_{q=1}^{l-1} X_{j q} R_{q l}^{(j)}
$$

Since $X_{j l}$ are bounded by $l_{n, \alpha}^{\frac{1}{\varkappa}}$, we may apply McDiarmid's inequality. This implies that

$$
\operatorname{Pr}\left\{\left|\alpha_{l}\right| \geq C \beta_{n}\left(\sum_{q \in \mathbb{T}_{j}}\left|R_{q l}^{(j)}\right|^{2}\right)^{\frac{1}{2}}\right\} \leq \exp \left\{-c \beta_{n}^{2} / l_{n, \alpha}^{\frac{2}{\varkappa}}\right\} \leq \exp \left\{-c l_{n, \alpha}\right\} .
$$

Now we consider the truncated martingale-difference

$\widehat{\alpha}_{l}=\alpha_{l} \mathbb{I}\left\{\left|\alpha_{l}\right| \leq C \beta_{n}\left(\sum_{q \in \mathbb{T}_{j}}\left|R_{q l}^{(j)}\right|^{2}\right)^{\frac{1}{2}}\right\}-\mathbf{E}\left\{\alpha_{l} \mathbb{I}\left\{\left|\alpha_{l}\right| \leq C \beta_{n}\left(\sum_{q \in \mathbb{T}_{j}}\left|R_{q l}^{(j)}\right|^{2}\right)^{\frac{1}{2}}\right\} \mid \mathfrak{M}_{l-1}^{(j)}\right\}$.

Applying McDiarmid's inequality for martingales (see [3]), we get

$$
\operatorname{Pr}\left\{\left|\sum_{l \in \mathbb{T}_{j}} \widehat{\alpha}_{l}\right| \geq x\left(\sum_{s, t \in \mathbb{T}_{j}}\left|R_{s t}^{(j)}\right|^{2}\right)^{\frac{1}{2}}\right\} \leq \exp \left\{-x^{2} / \beta_{n}^{2}\right\} .
$$

Putting $x=C \beta_{n}^{2}\left(\sum_{s, t \in \mathbb{T}_{j}}\left|R_{s t}^{(j)}\right|^{2}\right)^{\frac{1}{2}}$, we get

$$
\operatorname{Pr}\left\{\left|\sum_{l \in \mathbb{T}_{j}} \widehat{\alpha}_{l}\right| \geq \gamma_{n} \beta_{n} \sqrt{l_{n, \alpha}}\left(\sum_{s, t \in \mathbb{T}_{j}}\left|R_{s t}^{(j)}\right|^{2}\right)^{\frac{1}{2}}\right\} \leq \exp \left\{-c l_{n, \alpha}\right\} .
$$


Note that $\left|\alpha_{l}\right| \leq l_{n, \alpha}^{\frac{2}{\varkappa}} n v^{-1}$. Similar to (9.64) we get

$$
\begin{aligned}
\frac{1}{n} \sum_{l \in \mathbb{T}_{j}} \mathbf{E}\left\{\alpha _ { l } \mathbb { I } \left\{\left|\alpha_{l}\right|\right.\right. & \left.\left.\geq \beta_{n} \sqrt{l_{n, \alpha}}\left(\sum_{k \in \mathbb{T}_{j}}\left|R_{l k}^{(j)}\right|^{2}\right)^{\frac{1}{2}}\right\} \mid \mathfrak{M}_{l-1}^{(j)}\right\} \\
& \leq l_{n, \alpha}^{\frac{2}{\varkappa}} \exp \{2 \log n\} \exp \left\{-c l_{n, \alpha}\right\} \leq \exp \left\{-c^{\prime} l_{n, \alpha}\right\} .
\end{aligned}
$$

Inequalities (9.80), (9.83), and (9.85) together complete the proof of lemma. Thus Lemma 3.10 is proved.

\subsection{The Proof of Lemma 3.13}

We have

$$
\mathbf{E}\left\{\left|\widehat{\zeta}_{l}\right|^{m} \mathbb{I}\left\{\left(\mathcal{G}^{(j)}\right)^{c}\right\} \mid \mathfrak{M}^{(j)}\right\} \leq\left(\frac{C l_{n, \alpha}^{\frac{1}{\varkappa}} \sqrt{\psi_{0}}}{\sqrt{n v}}\right)^{m} \mathbb{I}\left\{\left(\mathcal{G}^{(j)}\right)^{c}\right\}
$$

and

$$
\mathbf{E}\left\{\left|\widehat{\zeta}_{l}^{(j)}\right|^{m} \mathbb{I}\left\{(\mathcal{G})^{c}\right\} \mid \mathfrak{M}^{(j)}\right\} \leq\left(\frac{C l_{n, \alpha}^{\frac{1}{\varkappa}} \sqrt{\psi_{0}}}{\sqrt{n v}}\right)^{m} \mathbb{I}\left\{\mathcal{G}^{(j)}\right\} \mathbf{E}\left\{\mathbb{I}\left\{\mathcal{G}^{c}\right\} \mid \mathfrak{M}^{(j)}\right\}
$$

Note that

$$
\mathcal{E}^{c} \cap \mathcal{E}=\emptyset, \quad \mathcal{U}^{c} \cap \mathcal{U}^{(j)}=\emptyset .
$$

From here it follows that

$$
\mathbb{I}\left\{\mathcal{G}^{(j)}\right\} \mathbf{E}\left\{\mathbb{I}\left\{\mathcal{G}^{c}\right\} \mid \mathfrak{M}^{(j)}\right\} \leq \mathbf{E}\left\{\mathbb{I}\left\{\mathcal{E}^{(j)} \cap \mathcal{U}^{(j)} \cap \mathcal{F}^{(j)} \cap \mathcal{F}^{c}\right\} \mid \mathfrak{M}^{(j)}\right\} .
$$

Note that

$$
\left|\zeta_{l}\right| \leq\left|\zeta_{l}^{(j)}\right|+\left|\zeta_{l}-\zeta_{l}^{(j)}\right|
$$

Therefore,

$$
\mathcal{F}_{l}^{c} \subset\left(\mathcal{F}_{l}^{(j)}\right)^{c} \cup\left\{\left|\zeta_{l}-\zeta_{l}^{(j)}\right| \geq \rho_{n}\left(\operatorname{Im} m_{n}^{(j)}(z)\right)^{\frac{1}{2}}\right\} .
$$

The relation (9.89) and (9.91) together imply

$$
\mathbb{I}\left\{\mathcal{F}^{c} \cap \mathcal{F}^{(j)}\right\} \leq \sum_{l \in \mathbb{T}_{j}} \mathbb{I}\left\{\left|\zeta_{l}-\zeta_{l}^{(j)}\right| \geq \rho_{n}\left(\operatorname{Im} m_{n}^{(j)}(z)\right)^{\frac{1}{2}}\right\} .
$$


Moreover,

$$
\begin{aligned}
\mathbb{I}\left\{\left|\zeta_{l}-\zeta_{l}^{(j)}\right| \geq \rho_{n}\left(\operatorname{Im} m_{n}^{(j)}(z)\right)^{\frac{1}{2}}\right\} \leq \mathbb{I}\left\{\left|\xi_{l j}\right|\right. & \left.\geq \frac{1}{2} \rho_{n}\left(\operatorname{Im} m_{n}^{(j)}(z)\right)^{\frac{1}{2}}\right\} \\
& +\mathbb{I}\left\{\left|\eta_{l j}\right| \geq \frac{1}{2} \rho_{n}\left(\operatorname{Im} m_{n}^{(j)}(z)\right)^{\frac{1}{2}}\right\} .
\end{aligned}
$$

Introduce the event

$$
\mathcal{P}:=\left\{\sum_{q \in \mathbb{T}_{j, l}}\left|R_{j q}^{(l)}\right|^{2} \leq \Gamma\left(C m^{2}\left(\operatorname{Im} m_{n}(z)+\frac{1}{n v}\right)+\Phi_{n}\right)\right\},
$$

for some constant $\Gamma>0$ which will be chosen later. Applying Chebyshev's inequality and Lemma 3.8 with $L=A m \log n$, where $A>0$ will be chosen later, we get

$$
\begin{aligned}
\mathbf{E}\left\{\mathbb{I}\left\{\mathcal{G}_{l}\right\} \mathbb{I}\left\{\left(\mathcal{G}_{l}^{(j)}\right)^{c}\right\}\right. & \left.\mathbb{I}\left\{\mathcal{P}^{c}\right\} \mid \mathfrak{M}^{(j)}\right\} \leq \mathbf{E}\left\{\mathbb{I}\left\{\mathcal{P}^{c}\right\} \mid \mathfrak{M}^{(j)}\right\} \\
& \leq \frac{\mathbf{E}\left\{\left(\sum_{q \in \mathbb{T}_{j}}\left|R_{j q}^{(l)}\right|^{2}\right)^{L} \mid \mathfrak{M}^{(j)}\right\}}{\Gamma^{L}\left(C m^{2}\left(\operatorname{Im} m_{n}^{(j)}(z)+\frac{1}{n v}\right)+C l_{n, \alpha}^{\frac{2}{\varkappa}}+\Phi_{n}\right)^{L}} \leq \frac{L^{2}}{\Gamma^{L}}
\end{aligned}
$$

Choosing appropriate constants $\Gamma>1$ and $A \geq 2 \log \Gamma$, we get

$$
\mathbf{E}\left\{\mathbb{I}\left\{\mathcal{G}_{l}^{c}\right\} \mathbb{I}\left\{\left(\mathcal{G}_{l}^{(j)}\right)\right\} \mathbb{I}\left\{\mathcal{P}^{c}\right\} \mid \mathfrak{M}^{(j)}\right\} \leq \frac{1}{n^{2 m}}
$$

Let

$$
V=\mathbf{E}\left\{\mathbb{I}\left\{\mathcal{U}^{(j)} \cap \mathcal{E}^{(j)} \cap\left\{\left|\xi_{l j}\right| \geq \frac{1}{2} \rho_{n}\left(\operatorname{Im} m_{n}^{(j)}(z)\right)^{\frac{1}{2}}\right\}\right\} \mathbb{I}\{\mathcal{P}\} \mid \mathfrak{M}^{(j)}\right\} .
$$

It is straightforward to check that

$$
V \leq \operatorname{Pr}\left\{\left|\xi_{l j}\right| \geq \frac{1}{2} \rho_{n}\left(\sqrt{\psi_{0}}\right)^{-1}\left(\sum_{q \in \mathbb{T}_{l, j}}\left|R_{j q}^{(l)}\right|^{2}\right) \mid \mathfrak{M}^{(j)}\right\} .
$$

Applying McDiarmid's inequality for martingale-differences similar to the proof of Lemma 3.10, we get

$$
\operatorname{Pr}\left\{\left|\xi_{l j}\right| \geq \frac{1}{2} \rho_{n}\left(\sqrt{\psi_{0}}\right)^{-1}\left(v \sum_{q \in \mathbb{T}_{l, j}}\left|R_{j q}^{(l)}\right|^{2}\right)\right\} \leq \exp \left\{-\sqrt{n v} / \sqrt{\kappa_{n}}\right\} .
$$

Similarly we get

$$
\operatorname{Pr}\left\{\left|\eta_{l j}\right| \geq \frac{1}{2} \rho_{n}\left(v \sum_{q \in \mathbb{T}_{l, j}}\left|R_{j q}^{(l)}\right|^{2}\right)^{\frac{1}{2}}\right\} \leq \exp \left\{-c \sqrt{n v} / \sqrt{\kappa_{n}}\right\} .
$$


Thus we have proved

$$
\left.\mathbf{E}\left\{\left|\widehat{\zeta}_{l}^{(j)}\right|^{m} \mathbb{I}\left\{(\mathcal{G})^{c}\right\}\right\} \mid \mathfrak{M}^{(j)}\right\} \leq\left(\frac{C l_{n, \alpha}^{\frac{2}{\varkappa}}}{\sqrt{n v}}\right)^{m} \Phi_{n} \leq \Phi_{n} .
$$

Inequalities (9.86), (9.87) and (9.96) together imply the claim. Thus Lemma 3.13 is proved.

Remark 9.2. As follows from (9.93) - (9.97) we have proved

$$
\mathbf{E}\left\{\mathbb{I}\left\{\mathcal{G}^{c}\right\} \mathbb{I}\left\{\left(\mathcal{G}^{(j)}\right)^{c}\right\} \mid \mathfrak{M}^{(j)}\right\} \leq \exp \left\{-c l_{n, \alpha}\right\}+\Phi_{n}
$$

\subsection{The proof of lemma 3.16}

For the simplicity we shall consider the case $\mathbb{A}=\emptyset$. In the other case we may consider matrix $\mathbf{W}^{(\mathbb{A})}$ instead of matrix $\mathbf{W}$. First we bound $\mathbf{E}\left\{\left|\widetilde{\alpha}_{l j}^{(\mathbb{A}, t)}\right|^{m} \mid \mathfrak{M}^{(\mathbb{A}, j, t)}\right\}$. By the definition of this quantity we have

$$
\begin{aligned}
\mathbf{E}\left\{\left|\widetilde{\alpha}_{l j}^{(t)}\right|^{m}\right. & \left.\mathbb{I} \mathcal{U}\} \mid \mathfrak{M}^{(j, t)}\right\} \\
& \leq\left(\frac{C l_{n, \alpha}^{\frac{1}{\varkappa}}}{n}\right)^{m} \mathbf{E}\left\{\left|\sum_{q \in \mathbb{T}_{l, j, t}} X_{l q} R_{q j}^{(l)}\right|^{m}\left|R_{t j}^{(l)}\right|^{m}\left|\left(R_{j j}^{(l)}\right)^{-1}\right|^{m} \mathbb{I}\{\mathcal{U}\} \mid \mathfrak{M}^{(j, t)}\right\} .
\end{aligned}
$$

Continue this inequality, applying representation (3.27). We obtain

$$
\begin{array}{r}
\mathbf{E}\left\{\left|\widetilde{\alpha}_{l j}^{(t)}\right|^{m} \mathbb{I}\{\mathcal{U}\} \mid \mathfrak{M}^{(j, t)}\right\} \leq\left(\frac{C l_{n, \alpha}^{\frac{1}{\varkappa}}}{\sqrt{n}}\right)^{m} \mathbf{E}\left\{\left|\frac{1}{\sqrt{n}}\left(\sum_{q \in \mathbb{T}_{l}} X_{l q} R_{q j}^{(l)}-X_{l j} R_{j j}^{(l)}-X_{l t} R_{t j}^{(l)}\right)\right|^{m}\right. \\
\left.\times\left|R_{t j}^{(l)}\right|^{m}\left|\left(R_{j j}^{(l)}\right)^{-1}\right|^{m} \mathbb{I}\{\mathcal{U}\} \mid \mathfrak{M}^{(j, t)}\right\} \\
\leq\left(\frac{C l_{n, \alpha}^{\frac{1}{\varkappa}}}{\sqrt{n}}\right)^{m} \mathbf{E}\left\{\left|R_{l j}\left(R_{j j}\right)^{-1}-\frac{1}{\sqrt{n}} X_{l j} R_{j j}^{(l)}-\frac{1}{\sqrt{n}} X_{l t} R_{t j}^{(l)}\right|^{m}\right. \\
\left.\times\left|R_{t j}^{(l)}\right|^{m}\left|\left(R_{j j}^{(l)}\right)^{-1}\right|^{m} \mathbb{I}\{\mathcal{U}\} \mid \mathfrak{M}^{(j, t)}\right\} .
\end{array}
$$


Applying representation (3.27) again and Corollary 3.5, we get

$$
\begin{aligned}
\mathbf{E}\left\{\left|\widetilde{\alpha}_{l j}^{(t)}\right|^{m} \mathbb{I}\{\mathcal{U}\} \mid \mathfrak{M}^{(j, t)}\right\} & \leq\left(\frac{C l_{n, \alpha}^{\frac{1}{\varkappa}}}{\sqrt{n}}\right)^{m} \mathbf{E}\left\{\left(\left|\frac{1}{\sqrt{n}} \sum_{q \in \mathbb{T}_{j}} X_{j q} R_{l q}^{(j)}\right|^{m}\right.\right. \\
& \left.+\left(\frac{l_{n, \alpha}^{\frac{1}{\varkappa}}}{\sqrt{n}}\right)^{m}\left(1+\left|\frac{1}{\sqrt{n}} \sum_{q \in \mathbb{T}_{l, j}} X_{j q} R_{t q}^{(j)}\right|^{m}\right)\right) \\
& \left.\times\left|\frac{1}{\sqrt{n}} \sum_{r \in \mathbb{T}_{l j}} X_{q j} R_{t q}^{(l, j)}\right|^{m} \mathbb{I}\{\mathcal{U}\} \mid \mathfrak{M}^{(j, t)}\right\} \\
& +\mathbf{E}\left\{\left|\widetilde{\alpha}_{l j}^{(t)}\right|^{m} \mathbb{I}\{\mathcal{U}\} \mathbb{I}\left\{\left(\mathcal{B} \cap \mathcal{B}^{(l)}\right)^{c}\right\} \mid \mathfrak{M}^{(j, t)}\right\} .
\end{aligned}
$$

This inequality implies that

$$
\begin{aligned}
\mathbf{E}\left\{\left|\widetilde{\alpha}_{l j}^{(t)}\right|^{m} \mathbb{I}\{\mathcal{U}\} \mid \mathfrak{M}^{(j, t)}\right\} \leq\left(\frac{C l_{n, \alpha}^{\frac{2}{\varkappa}}}{n}\right)^{m} \mathbf{E}\left\{\left(\frac{1}{\sqrt{n}}\left|\sum_{q \in \mathbb{T}_{l, j}} X_{j q} R_{t q}^{(j)}\right|\right)^{m} \mathbb{I}\{\mathcal{U}\} \mid \mathfrak{M}^{(j, t)}\right\} \\
+\left(\frac{C l_{n, \alpha}^{\frac{2}{\varkappa}}}{n}\right)^{m} \mathbf{E}\left\{\left(\frac{1}{\sqrt{n}}\left|\sum_{q \in \mathbb{T}_{l, j}} X_{j q} R_{t q}^{(j, l)}\right|\right)^{m}\left(\left|\frac{1}{\sqrt{n}} \sum_{q \in \mathbb{T}_{j}} X_{j q} R_{t q}^{(j)}\right|\right)^{m} \mathbb{I}\{\mathcal{U}\} \mid \mathfrak{M}^{(j, t)}\right\} \\
+\left(\frac{C l_{n, \alpha}^{\frac{1}{\varkappa}}}{\sqrt{n}}\right)^{m} \mathbf{E}\left\{\left(\frac{1}{\sqrt{n}}\left|\sum_{q \in \mathbb{T}_{l, j}} X_{j q} R_{t q}^{(j, l)}\right|\right)^{m}\left(\left|\frac{1}{\sqrt{n}} \sum_{q \in \mathbb{T}_{j}} X_{j q} R_{l q}^{(j)}\right|\right)^{m} \mathbb{I}\{\mathcal{U}\} \mid \mathfrak{M}^{(j, t)}\right\} \\
+\mathbf{E}\left\{\left|\widetilde{\alpha}_{l j}^{(t)}\right|^{m} \mathbb{I}\{\mathcal{U}\} \mathbb{I}\left\{\left(\mathcal{B B} \mathcal{B}^{(l)}\right)^{c}\right\} \mid \mathfrak{M}^{(j, t)}\right\} .
\end{aligned}
$$

Denote by $\widetilde{\mathcal{U}}^{(j, t)}:=\left\{m_{n}^{(j, t)}(z) \leq 2 \psi_{0}\right\}$. Using that $\mathbb{I}\{\mathcal{U}\} \leq \mathbb{I}\left\{\widetilde{\mathcal{U}}^{(j, t)}\right\}$ and 
applying Hölder's inequality and Rosenthal's inequality, we get

$$
\begin{aligned}
& \mathbf{E}\left\{\left|\widetilde{\alpha}_{l j}^{(t)}\right|^{m} \mathbb{I}\{\mathcal{U}\} \mid \mathfrak{M}^{(j, t)}\right\} \leq\left(\frac{C m l_{n, \alpha}^{\frac{3}{\varkappa}}}{n \sqrt{n}}\right)^{m} \mathbf{E}\left\{\left(\sum_{q \in \mathbb{T}_{l, j}}\left|R_{t q}^{(j)}\right|^{2}\right)^{\frac{m}{2}} \mathbb{I}\left\{\widetilde{\mathcal{U}}^{(j, t)}\right\} \mid \mathfrak{M}^{(j, t)}\right\} \\
& +\left(\frac{C m l_{n, \alpha}^{\frac{2}{\varkappa}}}{n}\right)^{2 m} \mathbf{E}^{\frac{1}{2}}\left\{\left(\sum_{q \in \mathbb{T}_{l, j}}\left|R_{t q}^{(j, l)}\right|^{2}\right)^{m} \mathbb{I}\left\{\widetilde{\mathcal{U}}^{(j, t)}\right\} \mid \mathfrak{M}^{(j, t)}\right\} \\
& \times \mathbf{E}^{\frac{1}{2}}\left\{\left(\sum_{q \in \mathbb{T}_{j}}\left|R_{t q}^{(j)}\right|^{2}\right)^{m} \mathbb{I}\left\{\widetilde{\mathcal{U}}^{(j, t)}\right\} \mid \mathfrak{M}^{(j, t)}\right\} \\
& +\left(\frac{C m l_{n, \alpha}^{\frac{2}{\varkappa}}}{n}\right)^{\frac{3}{2} m} \mathbf{E}^{\frac{1}{2}}\left\{\left(\sum_{q \in \mathbb{T}_{l, j}}\left|R_{t q}^{(j, l)}\right|^{2}\right)^{m} \mathbb{I}\left\{\widetilde{\mathcal{U}}^{(j, t)}\right\} \mid \mathfrak{M}^{(j, t)}\right\} \\
& \times \mathbf{E}^{\frac{1}{2}}\left\{\left(\sum_{q \in \mathbb{T}_{j}}\left|R_{l q}^{(j)}\right|^{2}\right)^{m} I\left\{\widetilde{\mathcal{U}}^{(j, t)}\right\} \mid \mathfrak{M}^{(j, t)}\right\}+\mathbf{E}\left\{\left|\widetilde{\alpha}_{l j}^{(t)}\right|^{m} \mathbb{I}\{\mathcal{U}\} \mathbb{I}\left\{\left(\mathcal{B B}^{(l)}\right)^{c}\right\} \mid \mathfrak{M}^{(j, t)}\right\} .
\end{aligned}
$$

This inequality and Lemmas 3.8 and 3.9 together imply

$$
\begin{gathered}
\mathbf{E}\left\{\left|\widetilde{\alpha}_{l j}^{(t)}\right|^{m} \mathbb{I}\{\mathcal{U}\} \mid \mathfrak{M}^{(j, t)}\right\} \leq\left(\frac{C m^{\frac{3}{2}} l_{n, \alpha}^{\frac{3}{\varkappa}}}{n \sqrt{n v}}\right)^{m} \psi_{0}^{\frac{m}{2}}+\left(\frac{C m^{2} l_{n, \alpha}^{\frac{4}{\varkappa}}}{n^{2} v}\right)^{m} \psi_{0}^{m}+\Phi_{n} \\
+\left(\frac{C m^{\frac{3}{2}} l_{n, \alpha}^{\frac{3}{\varkappa}}}{(n v) \sqrt{n}}\right)^{m} \psi_{0}^{\frac{m}{2}}\left(v \sum_{q \in \mathbb{T}_{j, t, l}}\left|R_{q l}^{(j, t)}\right|^{2}\right)^{\frac{m}{2}}\left(\left(v \sum_{q \in \mathbb{T}_{j, t, l}}\left|R_{q l}^{(j, t)}\right|^{2}\right)^{\frac{m}{2}}+\left(\frac{C m l_{n, \alpha}^{\frac{2}{\varkappa}}}{\sqrt{n}}\right)^{m}\right) \\
\times \mathbb{I}\left\{\widetilde{\mathcal{U}}^{(j, t)}\right\} .
\end{gathered}
$$

Using that $\psi_{0} \geq c \sqrt{v}$, we may write

$$
\begin{aligned}
& \mathbf{E}\left\{\left|\widetilde{\alpha}_{l j}^{(t)}\right|^{m} \mathbb{I}\{\mathcal{U}\} \mid \mathfrak{M}^{(j, t)}\right\} \leq\left(\frac{C m^{\frac{3}{2}} l_{n, \alpha}^{\frac{3}{\varkappa}}}{(n v) \sqrt{n v}}\right)^{m} \psi_{0}^{\frac{3 m}{2}}+\Phi_{n} \\
& +\left(\frac{C m^{\frac{3}{2}} l_{n, \alpha}^{\frac{3}{\varkappa}}}{(n v) \sqrt{n}}\right)^{m} \psi_{0}^{\frac{m}{2}}\left(v \sum_{q \in \mathbb{T}_{j, t, l}}\left|R_{q l}^{(j, t)}\right|^{2}\right)^{m} I\left\{\widetilde{\mathcal{U}}^{(j, t)}\right\} .
\end{aligned}
$$

We consider now $\widehat{\alpha}_{\nu l j}^{(\mathbb{A}, t)}$, for $\nu=1,2,3$. We consider $\nu=1$ only. The 
other cases are similar. Using equality (3.27), we write

$$
\begin{aligned}
\widehat{\alpha}_{1 l j}^{(t)} & =\frac{1}{\sqrt{n}} \sum_{r \in \mathbb{T}_{l, t}} X_{l r} R_{j r}^{(l)} \frac{1}{\sqrt{n}} \sum_{q \in \mathbb{T}_{l, t, r}} X_{l q} R_{q t}^{(l)} R_{j t}^{(l)}\left(R_{j j}^{(l)}\right)^{-1}\left(R_{t t}^{(l)}\right)^{-1} \\
& =\frac{1}{\sqrt{n}} \sum_{r \in \mathbb{T}_{l, t}} X_{l r} R_{j r}^{(l)}\left(R_{l t} R_{l l}^{-1}-\frac{1}{\sqrt{n}} X_{l r} R_{t r}^{(l)}-\frac{1}{\sqrt{n}} X_{l t} R_{t t}^{(l)}\right)\left(R_{j j}^{(l)}\right)^{-1}\left(R_{t t}^{(l)}\right)^{-1} R_{j t}^{(l)} \\
& =\left(R_{l j} R_{l l}^{-1}-\frac{1}{\sqrt{n}} X_{l t} R_{j t}^{(l)}\right)\left(R_{l t} R_{l l}^{-1}-\frac{1}{\sqrt{n}} X_{l t} R_{t t}^{(l)}\right)\left(R_{j j}^{(l)}\right)^{-1} R_{j t}^{(l)}\left(R_{t t}^{(l)}\right)^{-1} \\
& -\frac{1}{n} \sum_{r \in \mathbb{T}_{l, t}} X_{l r}^{2} R_{j r}^{(l)} R_{t r}^{(l)} R_{j t}^{(l)}\left(R_{j j}^{(l)}\right)^{-1}\left(R_{t t}^{(l)}\right)^{-1}
\end{aligned}
$$

For $\omega \in \mathcal{B}^{(l)}$, we have

$$
\begin{array}{r}
\left|\widehat{\alpha}_{1 l j}^{(t)}\right| \leq C\left(\left|R_{l t}\right|^{2}+\left|R_{l j}\right|^{2}+\left(\left|R_{j t}^{(l)}\right|^{2}+\left|R_{j t}^{(l)}\right|\right) \frac{C l_{n, \alpha}^{\frac{2}{\varkappa}}}{n}\right)\left|R_{j t}^{(l)}\right| \\
+\frac{C l_{n, \alpha}^{\frac{2}{\varkappa}}}{n}\left(\sum_{r \in \mathbb{T}_{l, t}}\left|R_{j r}^{(l)}\right|^{2}+\sum_{r \in \mathbb{T}_{l, t}}\left|R_{t r}^{(l)}\right|^{2}\right)\left|R_{j t}^{(l)}\right| .
\end{array}
$$

Applying the representation (3.27), we get for $\omega \in \mathcal{B} \cap \mathcal{B}^{(l)}$,

$$
\begin{aligned}
\left|\widehat{\alpha}_{1 l j}^{(t)}\right| & \leq \frac{C}{n^{\frac{3}{2}}}\left(\left|\sum_{q \in T_{t}} X_{t q} R_{q l}^{(t)}\right|^{2}+\left|\sum_{q \in T_{j}} X_{j q} R_{q l}^{(j)}\right|^{2}\right)\left|\sum_{q \in \mathbb{T}_{j, l}} X_{j q} R_{q t}^{(j, l)}\right| \\
& +\frac{C l_{n, \alpha}^{\frac{2}{\varkappa}}}{n^{\frac{5}{2}}}\left(\left|\sum_{q \in \mathbb{T}_{j, l}} X_{j q} R_{q t}^{(j, l)}\right|^{3}+\left|\sum_{q \in \mathbb{T}_{j, l}} X_{j q} R_{q t}^{(j, l)}\right|^{2}\right) \\
& +\frac{C l_{n, \alpha}^{\frac{2}{\varkappa}}}{n^{\frac{3}{2}}}\left(\sum_{r \in \mathbb{T}_{l, t}}\left|R_{j r}^{(l)}\right|^{2}+\sum_{r \in \mathbb{T}_{l, t}}\left|R_{t r}^{(l)}\right|^{2}\right)\left|\sum_{q \in \mathbb{T}_{j, l}} X_{j q} R_{q t}^{(j, l)}\right| .
\end{aligned}
$$


This inequality, Lemma 3.8 and McDiarmid's inequality together imply

$$
\begin{aligned}
& \mathbf{E}^{\frac{1}{m}}\left\{\left|\widehat{\alpha}_{1 l j}^{(t)}\right|^{m} \mathbb{I}\{\mathcal{U}\} \mid \mathfrak{M}^{(t, j)}\right\} \leq\left(\frac{C l_{n, \alpha}^{\frac{1}{\varkappa}}}{\sqrt{n v}}\right)^{3}\left(\left(\mathbf{E}^{\frac{2}{3 m}}\left\{\left(v \sum_{q \in \mathbb{T}_{t}}\left|R_{q l}^{(t)}\right|^{2}\right)^{\frac{3 m}{2}} \mathbb{I}\{\mathcal{U}\} \mid \mathfrak{M}^{(j, t)}\right\}\right.\right. \\
& \left.\quad+\mathbf{E}^{\frac{2}{3 m}}\left\{\left(v \sum_{q \in \mathbb{T}_{j}}\left|R_{q l}^{(j)}\right|^{2}\right)^{\frac{3 m}{2}} \mathbb{I}\{\mathcal{U}\} \mid \mathfrak{M}^{(t, j)}\right\}\right) \mathbf{E}^{\frac{1}{3 m}}\left\{\left(v \sum_{q \in \mathbb{T}_{j, l}}\left|R_{q t}^{(j)}\right|^{2}\right)^{\frac{3 m}{2}} \mathbb{I}\{\mathcal{U}\} \mid \mathfrak{M}^{(t, j)}\right\} \\
& \quad+\mathbf{E}^{\frac{1}{m}}\left\{\left(v \sum_{q \in \mathbb{T}_{j, l}}\left|R_{q t}^{(j)}\right|^{2}\right)^{\frac{3 m}{2}} \mathbb{I}\{\mathcal{U}\} \mid \mathfrak{M}^{(t, j)}\right\}+\mathbf{E}^{\frac{1}{m}}\left\{\left(v \sum_{q \in \mathbb{T}_{j, l}}\left|R_{q t}^{(j)}\right|^{2}\right)^{m} \mathbb{I}\{\mathcal{U}\} \mid \mathfrak{M}^{(t, j)}\right\} \\
& +\left(\mathbf{E}^{\frac{2}{3 m}}\left\{\left(\sum_{r \in \mathbb{T}_{l, t}}\left|R_{j r}^{(l)}\right|^{2}\right)^{\frac{3 m}{2}} \mathbb{I}\{\mathcal{U}\} \mid \mathfrak{M}^{(j)}\right\}+\mathbf{E}^{\frac{2}{3 m}}\left\{\left(\sum_{r \in \mathbb{T}_{l, t}}\left|R_{t r}^{(l)}\right|^{2}\right)^{\frac{3 m}{2}} \mathbb{I}\{\mathcal{U}\} \mid \mathfrak{M}^{(j)}\right\}\right) \\
& \left.\quad \times \mathbf{E}^{\frac{1}{3 m}}\left\{\left(v \sum_{q \in \mathbb{T}_{j l}}\left|R_{q t}^{(j, l)}\right|^{2}\right)^{\frac{3 m}{2}} \mathbb{I}\{\mathcal{U}\} \mid \mathfrak{M}^{(j, l)}\right\}+n^{3} \mathbf{E}\left\{\mathbb{I}\left\{\mathcal{U} \cap\left(\mathcal{B} \cap \mathcal{B}^{(l)}\right)^{c}\right\} \mid \mathfrak{M}^{(j, t)}\right\}\right) .
\end{aligned}
$$

Using Lemmas 3.8 and 3.9, we get

$$
\begin{aligned}
& \mathbf{E}^{\frac{1}{m}}\left\{\left|\widehat{\alpha}_{1 l j}^{(t)}\right|^{m} \mid \mathfrak{M}^{(t, j)}\right\} \leq \frac{C m^{3} l_{n, \alpha}^{\frac{3}{\varkappa}}}{(n v)^{\frac{3}{2}}}\left(v\left(\sum_{q \in \mathbb{T}_{t}}\left|R_{q l}^{(j, t)}\right|^{2}\right) \psi_{0}^{\frac{1}{2}} \mathbb{I}\left\{\mathcal{U}^{(j, t)}\right\}+\psi_{0}^{\frac{3}{2}}\right. \\
& \left.+n^{3} \mathbf{E}\left\{\mathbb{I}\left\{\mathcal{U} \cap\left(\mathcal{B} \cap \mathcal{B}^{(l)}\right)^{c}\right\} \mid \mathfrak{M}^{(j, t)}\right\}\right) .
\end{aligned}
$$

Thus Lemma 3.16 is proved.

\subsection{Proof of Lemma 3.18}

The proof is similar to the proof of Lemma 3.16. We shall consider the case $\mathbb{A}=\emptyset$ only. First we represent

$$
\gamma_{l j}^{(t)}=\widetilde{\gamma}_{j l}^{(t)}+\widehat{\gamma}_{j l}^{(t)}
$$

where

$$
\begin{aligned}
\widetilde{\gamma}_{l j}^{(t)} & =\frac{1}{n} X_{l j} X_{l t} R_{j t}^{(l)} \\
\widehat{\gamma}_{l j}^{(t)} & =\frac{1}{n} X_{l j} \sum_{q \in \mathbb{T}_{l, j, t}} X_{l q} R_{q t}^{(l)} R_{j t}^{(l)}\left(R_{t t}^{(l)}\right)^{-1}
\end{aligned}
$$


Note that

$$
\mathbf{E}^{\frac{1}{m}}\left\{\left(\widetilde{\gamma}_{l j}^{(t)}\right)^{m} \mathbb{I}\{\mathcal{U}\} \mid \mathfrak{M}^{(j, t)}\right\} \leq \frac{C l_{n, \alpha}^{\frac{2}{\varkappa}}}{n} \mathbf{E}^{\frac{1}{m}}\left\{\left|R_{j t}^{(l)}\right|^{m} \mathbb{I}\{\mathcal{U}\} \mid \mathfrak{M}^{(j, t)}\right\}
$$

Applying the representation (3.27) and McDiarmid's inequality, we obtain $\mathbf{E}^{\frac{1}{m}}\left\{\left(\widetilde{\gamma}_{l j}^{(t)}\right)^{m} \mathbb{I}\{\mathcal{U}\} \mid \mathfrak{M}^{(j, t)}\right\} \leq \frac{C l_{n, \alpha}^{\frac{2}{\varkappa}}}{n \sqrt{n}} \mathbf{E}^{\frac{1}{m}}\left\{\left(\sum_{q \in \mathbb{T}_{j, l, t}}\left|R_{q t}^{(l, j)}\right|^{2}\right)^{\frac{m}{2}} \mathbb{I}\{\mathcal{U}\} \mid \mathfrak{M}^{(j, t)}\right\}+\Phi_{n}$.

Applying Lemma 3.8 now, we get

$$
\mathbf{E}^{\frac{1}{m}}\left\{\left(\widetilde{\gamma}_{l j}^{(t)}\right)^{m} \mathbb{I}\{\mathcal{U}\} \mid \mathfrak{M}^{(j, t)}\right\} \leq \frac{C \sqrt{\kappa_{n}}}{n \sqrt{n v}}+\Phi_{n} .
$$

Taking in account that $\psi_{0} \geq c \sqrt{v}$, we may write

$$
\mathbf{E}^{\frac{1}{m}}\left\{\left(\widetilde{\gamma}_{l j}^{(t)}\right)^{m} \mathbb{I}\{\mathcal{U}\} \mid \mathfrak{M}^{(j, t)}\right\} \leq \frac{C \kappa_{n}^{\frac{3}{2}}}{n v \sqrt{n v}}+\Phi_{n}
$$

Re-expanding $\widehat{\gamma}_{l j}^{(t)}$ in jth row, we obtain for $\omega \in \mathcal{B}^{(l)} \cap \mathcal{B}$,

$$
\left|\widehat{\gamma}_{l j}^{(t)}\right| \leq \frac{C l_{n, \alpha}^{\frac{1}{\varkappa}}}{\sqrt{n}}\left(\left|R_{l t}\right|+\frac{1}{\sqrt{n}}\left|X_{l t}\right|+\frac{1}{\sqrt{n}}\left|X_{l j}\right|\left|R_{j t}^{(l)}\right|\right)\left|R_{j t}^{(l)}\right| .
$$

The last inequality yields

$$
\begin{aligned}
\mathbf{E}^{\frac{1}{m}}\left\{\left|\widehat{\gamma}_{l j}^{(t)}\right|^{m} \mathbb{I}\{\mathcal{U}\} \mid \mathfrak{M}^{(j, t)}\right\} \leq & \frac{C l_{n, \alpha}^{\frac{1}{\varkappa}}}{n \sqrt{n}} \mathbf{E}^{\frac{1}{2 m}}\left\{\left(\sum_{q \in \mathbb{T}_{l}}\left|R_{q l}^{(t)}\right|^{2}\right)^{m} \mathbb{I}\{\mathcal{U}\} \mid \mathfrak{M}^{(j, t)}\right\} \\
& \times \mathbf{E}^{\frac{1}{2}}\left\{\left(\sum_{q \in \mathbb{T}_{l}}\left|R_{q t}^{(j)}\right|^{2}\right)^{m} \mathbb{I}\{\mathcal{U}\} \mid \mathfrak{M}^{(j, t)}\right\}+\Phi_{n} .
\end{aligned}
$$

Applying Lemmas 3.8 and 3.9, we get

$$
\mathbf{E}^{\frac{1}{m}}\left\{\left|\widehat{\gamma}_{l j}^{(t)}\right|^{m} \mathbb{I}\{\mathcal{U}\} \mid \mathfrak{M}^{(j, t)}\right\} \leq \frac{C \sqrt{\kappa_{n}}}{n \sqrt{n v}}\left(\sum_{q \in \mathbb{T}_{j, l, t}}\left|R_{q l}^{(j, t)}\right|^{2}\right)^{\frac{1}{2}} \mathbb{I}\left\{\mathcal{U}^{(j, t)}\right\}+\Phi_{n} .
$$

Thus the Lemma is proved.

Acknowledgement. The authors would like to thank S. G. Bobkov for drawing their attention to some references about large deviations for martingales and quadratic forms. 


\section{References}

[1] Bai, Z. D., Miao, Tsay, J. Convergence rates of the spectral distributions of large Wigner matrices. Int. Math. J. 1 (2002), 65-90.

[2] Bai, Z. D. Convergence rate of expected spectral distributions of large random matrices. I. Wigner matrices. Ann. Probab. 21 (1993), no. 2, 625648 .

[3] Bentkus, V. Measure concentration for separately Lipschitz functions in product spaces . Israel Journal of Mathematics, 158 (2007), 1-17.

[4] Bobkov S. G. Some extremal properties of the Bernoulli distribution Teor. Veroyatnost. i Primenen. 41 (1996), no. 4, 877-884; translation in Theory Probab. Appl. 41 (1996), no. 4, 748755 (1997).

[5] Bobkov, S. G. Some generalizations of the results of Yu. V. Prokhorov on Khinchin-type inequalities for polynomials. (Russian) Teor. Veroyatnost. $i$ Primenen. 45 (2000), no. 4, 745-748; translation in Theory Probab. Appl. 45 (2002), no. 4, 644647

[6] Bobkov, S. G.; Gtze, F. Exponential integrability and Transportation Cost Related to Logarithmic Sobolev Inequalities. Journal of Functional Analysis, 163 (1999), 1- 28.

[7] Bobkov, S.; Götze, F.; Tikhomirov, A. N. On concentration of empirical measures and convergence to the semi-circle law. Journal of Theoretical Probability, 23, (2010), 792-823.

[8] Girko, V. L. Extended proof of the statement: Convergence rate of expected spectral functions of symmetric random matrices $\Sigma_{n}$ is equal $O\left(n^{-\frac{1}{2}}\right)$ and the method of critical steepest descent. Random Oper. Stochastic Equations 10 (2002), 253-300.

[9] Götze, F.; Tikhomirov, A. N. The rate of convergence of spectra of sample covariance matrices. Teor. Veroyatn. Primen. 54 (2009), 196206.

[10] Götze, F.; Tikhomirov, A. N. The rate of convergence for spectra of GUE and LUE matrix ensembles. Cent. Eur. J. Math. 3 (2005), 666704.

[11] Götze, F.; Tikhomirov, A. N. Rate of convergence to the semi-circular law. Probab. Theory Relat. Fields 127 (2003), 228-276. 
[12] Gustavsson, Jonas. Gaussian fluctuations of eigenvalues in the GUE. Ann. I. H. Poincare -PR 41 (2005), 151-178.

[13] Erdös, L.; Yau; H.-T, Yin, J. Bulk universality for generalized Wigner matrices. Preprint, arXiv:1001.3453.

[14] Erdös, L.; Yau, H.-T.; Yin, J. Rigidity of eigenvalues of generalized Wigner matrices. Preprint, arXiv:1007.4652.

[15] Timushev, D. A.; Tikhomirov, A. N.; Kholopov, A. A. On the accuracy of the approximation of the GOE spectrum by the semi-circular law. Teor. Veroyatn. Primen. 52 (2007), no. 1, 180-185.

[16] Tikhomirov, A. N. On the rate of convergence of the expected spectral distribution function of a Wigner matrix to the semi-circular law. Siberian Adv. Math. 19, (2009), 211-223. 